\title{
A new Monte Carlo code for star cluster simulations
}

\section{Central black hole and stellar collisions}

\author{
M. Freitag 1,2 and W. Benz ${ }^{2}$ \\ 1 California Institute of Technology, Mail code 130-33, Pasadena, CA 91125, USA \\ 2 Physikalisches Institut, Universität Bern, Sidlerstrasse 5, 3012 Bern, Switzerland \\ Received 17 April 2002 / Accepted 31 July 2002

\begin{abstract}
We have recently written a new code to simulate the long term evolution of spherical clusters of stars. It is based on the pioneering Monte Carlo scheme proposed by Hénon in the 70's. Unlike other implementations of this numerical method which were successfully used to investigate the dynamics of globular clusters, our code has been devised in the specific goal to treat dense galactic nuclei. In a previous paper, we described the basic version of our code which includes 2-body relaxation as the only physical process. In the present work, we go on and include further physical ingredients that are mostly relevant to galactic nuclei, namely the presence of a central (growing) black hole (BH) and collisions between (main sequence) stars. Stars that venture too close to the $\mathrm{BH}$ are destroyed by the tidal field. We took particular care of this process because of its importance, both as a channel to feed the $\mathrm{BH}$ and a way to produce accretion flares from otherwise quiescent galactic nuclei. Collisions between stars have often been proposed as another mechanism to drive stellar matter into the central $\mathrm{BH}$. Furthermore, non disruptive collisions may create peculiar stellar populations which are of great observational interest in the case of the central cluster of our Galaxy. To get the best handle on the role of this process in galactic nuclei, we include it with unpreceded realism through the use of a set of more than 10000 collision simulations carried out with a SPH (Smoothed Particle Hydrodynamics) code. Stellar evolution has also been introduced in a simple way, similar to what has been done in previous dynamical simulations of galactic nuclei. To ensure that this physics is correctly simulated, we realized a variety of tests whose results are reported here. This unique code, featuring most important physical processes, allows million particle simulations, spanning a Hubble time, in a few CPU days on standard personal computers and provides a wealth of data only rivalized by $N$-body simulations.
\end{abstract}

Key words. methods: numerical - galaxies: stellar dynamics - galaxies: nuclei - galaxies: star clusters

\section{Introduction}

This paper is the second part of the description of the code we have developed in the past few years in order to investigate the long-term dynamics of dense galactic nuclei. In a first paper (Freitag \& Benz 2001, hereafter paper I), we presented the basic version of this Monte Carlo (MC) code which deals with 2-body relaxation. In this article, we add flesh to this kernel by incorporating physical effects that are of particular interest and relevance for galactic nuclei.

The structure of the paper is as follows. In Sect. 1.1, we motivate our interest in the dynamics of galactic nuclei through a short review of the history of the study of this field. We then proceed to describe the new physics incorporated in the code. The principles of the basic version of our MC code are presented in Paper I with which the reader is advised to get familiar; we don't repeat this information here. Our treatment of stellar collisions is treated in Sect. 2, tidal disruptions in Sect. 3,

Send offprint requests to: M. Freitag, e-mail: freitag@tapir.caltech.edu while further, more minor, additions and improvements are described in Sect. 4. A variety of test simulations are reported and discussed in Sect. 5. Finally we summarize this work and propose future developments in Sect. 6. An Appendix is added to expose how we build initial conditions for use with our code.

\subsection{Astrophysical motivation}

Only very few reviews have been written about the dynamics of galactic nuclei (Gerhard 1994 is the only recent reference known to us), hence, we introduce our work by a summary of the history of this complex field.

The theoretical study of the dynamics of galactic nuclei was initiated in the 60 's, mostly to investigate whether stellar collisions in extremely dense clusters could power the, then recently discovered, quasars (QSOs). In these early speculations, the presence of a central massive black hole (MBH) wasn't assumed. QSO observations required the concentration of a huge amount of matter in a small volume and it was thought that a stellar system could expel its angular momentum and 
contract more easily than a purely gaseous configuration, hence reaching densities such that highly energetic collisions between stars should be commonplace (Gold et al. 1965; von Hoerner 1968). While Woltjer (1964) proposed that collisions themselves would be a strong source of optical radiation and radio-emitting energetic particles, others pointed out that these disruptive events should lead to the formation of a massive compact gas object (Ulam \& Walden 1964), maintained at QSO luminosity either by further collisions (Spitzer \& Saslaw 1966; Spitzer \& Stone 1967) or massive star formation and supernovae (SN) explosions (Sanders 1970). Growth of high-mass stars through repeated mergers in a low-velocity collisional cluster was proposed by Colgate (1967) as another way of forming SN-powered QSOs. Unfortunately, in none of these early studies, was the stellar dynamics treated in a realistic way, most authors having recourse to some extension of the evaporative model of globular clusters (see, e.g., Spitzer 1987). In particular, the process of gravothermal collapse was not known and the role of mass segregation not properly recognized.

Nearly all further studies accounted for the presence of a central $\mathrm{MBH}$, an object more and more widely accepted as necessary to explain QSOs and others Active Galactic Nuclei (AGN), and likely to be present in at least some normal presentday nuclei, as a relic of past activity (Lynden-Bell 1969). In a relaxed cluster where stars are destroyed in the vicinity of the BH, presumably by tidal forces (Hills 1975), their (quasi-)stationary density distribution was predicted to be a power-law, $n_{*} \propto R^{-7 / 4}$ (Peebles 1972; Bahcall \& Wolf 1976; Bahcall \& Wolf 1977). The tidal disruption rate is dominated by stars that are brought onto very elongated orbits by relaxation (Frank \& Rees 1976; Lightman \& Shapiro 1977; Young et al. 1977; Cohn \& Kulsrud 1978, see Sect. 3.1). This rate is probably insufficient to feed a QSO-class MBH unless the stellar density is so high that collisional gas production should dominate (Young et al. 1977; Young 1977; Shields \& Wheeler 1978; Frank 1978). The link between the earlier BH-free stellar dynamical models for AGN and these studies of MBH-cluster systems was traced by Begelman \& Rees (1978) who showed that most very dense stellar systems will naturally evolve to form large BHs.

In the 80 's, self-consistent simulations of the evolution of galactic nuclei appeared, that confirmed these conclusions (McMillan et al. 1981; Duncan \& Shapiro 1983). These models were based on Fokker-Planck and Monte Carlo codes first developed to study globular clusters. A serious shortcoming of these works was to assume that collisions were completely disruptive. David et al. (1987a,b) and Murphy et al. (1991) improved on this by implementing some extension of the simple semi-analytical prescription of Spitzer \& Saslaw (1966) to account for partial disruptions but the introduction of collisions into Fokker-Planck codes had to be done in a quite unrealistic way (see Sect. 5.3). Stellar evolution was also included with the conclusion that, provided a significant fraction of the emitted gas is accreted, it dominates the feeding of the $\mathrm{BH}$ in systems of moderate stellar density while collisions are still the main player in denser nuclei and that the full range of AGN and QSO luminosities can be attained without having recourse to an external source of gas. More recently, Rauch (1999) has considered the relativistic dynamics of a compact stellar cluster dominated by a central $\mathrm{MBH}$ in an AGN and concluded that collisions, most of which are grazing, produce only little gas but may efficiently replenish the loss-cone (see Sect. 3.1) for tidal disruptions.

In the past decade, gas-dynamical processes have been increasingly favored over stellar dynamics as the main source of fueling of AGN (Shlosman et al. 1990; Shlosman 1992; Combes 2001, and references therein). It is argued that, to achieve the highest QSO luminosities, the initial stellar cluster has to be so dense that its formation is problematic and would, most likely, require to concentrate a large amount of gas in the galactic center anyway. Furthermore, whether most of the gas emitted by stars - either in the course of their normal evolution or through collisions - finds its way to the $\mathrm{MBH}$ is uncertain (see Sect. 6.2). However, it may have been overlooked that the effective stellar relaxation rate, and, hence $\mathrm{BH}$ fueling through tidal disruptions or direct horizon crossings, may be highly enhanced by small departures from the assumption of a smooth spherical potential. Such departures may be the presence of orbiting cores or nuclear BHs of smaller accreted galaxies (Polnarev \& Rees 1994; Zhao et al. 2002), or triaxiality (Norman \& Silk 1983) which may survive in the vicinity of the $\mathrm{BH}$ even if it is destroyed at intermediate scales (Poon \& Merritt 2002) ${ }^{1}$.

Even though purely stellar dynamical processes are probably only secondary in feeding QSO-class MBHs, they may be efficient enough to grow few million solar masses objects from BHs with a mass of a few hundreds $M_{\odot}$. Furthermore, questions regarding the interplay between the stellar nucleus and a central $\mathrm{MBH}$ are more pressing than ever, as observational evidences for the presence of MBHs in most, if not all, bright galaxies, including the Milky Way, are accumulating at an impressive rate (Ferrarese et al. 2001; Merritt \& Ferrarese 2001; Kormendy \& Gebhardt 2001; Genzel et al. 2000; Ghez et al. 2000, and references in Paper I). In particular, tidal disruptions at a rate of order $10^{-4} \mathrm{yr}^{-1}$ seem unavoidable for BHs less massive than a few $10^{8} M_{\odot}$, with the likely consequence of bringing back to active life an otherwise quiescent galactic nucleus (Hills 1975; Lidskii \& Ozernoi 1979; Rees 1988; Phinney 1989; Sembay \& West 1993; Syer \& Ulmer 1999; Magorrian \& Tremaine 1999). Ironically, while tidal disruptions are deemed too rare to be the main contributor to the growth of MBHs, even in very dense nuclei, they are predicted in present-day normal nuclei with a rate which is embarrassingly high in regard to the low luminosity of these objects, a fact that has been used to impose constraints on gas accretion models (Sanders \& van Oosterom 1984; Menou \& Quataert 2001). Some flaring events in the UV or X-ray band from the center of active and non-active galaxies have been tentatively interpreted as the accretional aftermath of tidal disruptions (Greiner et al. 2000; Komossa 2001; Renzini 2001, and references therein). But further conclusions have to await more complete stellar dynamical simulations, like the ones we propose to carry out with our code, and a better

\footnotetext{
Unfortunately, such possibilities, although pointing to the importance of stellar dynamical processes, could only be introduced approximately in our code which relies on spherical symmetry.
} 
understanding of the post-disruption accretion process in order to predict its observational signature (wavelength, intensity, duration, etc.) Beside the accretion flares, another promising observational consequence is predicted: the production of hot, very bright, stellar cores of tidally stripped giant stars (Di Stefano et al. 2001).

As recalled above, collisions were first thought to play a major role in very dense nuclei models, either by feeding the $\mathrm{MBH}$ or by directly producing the AGN luminosity. The latter class of models, now incorporating a central $\mathrm{BH}$, has been revived by Torricelli-Ciamponi et al. (2000) but should be examined in the light of a more refined treatment of stellar collisions and stellar dynamics. Even if they are not frequent enough to have a strong impact on the dynamics or $\mathrm{BH}$ fueling, collisions may have interesting observational consequences, by producing peculiar stellar populations, like blue stragglers (Sills et al. 2001, and references therein, in the context of globular clusters), or destroying giant stars (Genzel et al. 1996; Alexander 1999; Bailey \& Davies 1999), for instance.

In addition to the now almost "classical" questions concerning tidal disruptions and collisions, the stellar dynamics of galactic nuclei is key in other processes of high observational importance. An important example is capture of compact stars on relativistic orbits around the $\mathrm{MBH}$. Through relaxation or collisions, a compact star may get on a very elongated orbit with such a small pericenter distance that emission of gravitational waves will drive further orbital evolution until the star plunges through the horizon of the MBH (Hils \& Bender 1995; Sigurdsson \& Rees 1997; Freitag 2001; Ivanov 2001). As these waves, if successfully detected and analyzed, would be a direct probe to the space-time geometry near MBHs (Thorne 1998; Hughes 2001a,b), such relativistic MBH-star binaries will be prime-interest sources for the future space-borne laser interferometer LISA (Danzmann 2000). This question and other ones to be mentioned in Sect. 6.2 are beyond the scope of this paper and the relevant physics are not included in the code described here (see, however, Freitag 2001, for our first results concerning the capture of compact objects). Nonetheless, they strongly motivate the need for detailed numerical models of the stellar dynamics in the center-most parts of galaxies.

\subsection{General approach and limitations}

As is clear from this introduction and was already stressed in Paper I, the physics of galactic nuclei is a very intricate problem, with dozens of physical processes or aspects that can potentially play a role and interfere with each other. Any really general and realistic approach would have to face too many computational challenges and unknowns concerning the physics, initial and limit conditions to be feasible at the present date. Various numerical methods have different limitations and require different simplifying assumptions which delineate the class of models that can be treated.

For instance, it is increasingly recognized that galaxy merging is a common process in the universe and that such events have deep imprint on the structure of galactic nuclei (Nakano \& Makino 1999; Merritt \& Cruz 2001). Of particular interest is the formation and evolution of binary BHs formed in the process (Begelman et al. 1980; Gould \& Rix 2000; Hemsendorf et al. 2001; Milosavljević \& Merrit 2001; Yu 2002). Selfconsistent simulation of these highly dynamical episodes in the life of galactic nuclei can only be done with $N$-body codes in which the orbits of $N$ particles are explicitly integrated for many dynamical times. However, such direct $N$-body integrations are extraordinarily CPU-demanding and, when various physical processes interplay whose relative importance depends on $N$, their results can not be safely scaled to $N \gg 10^{6}$ to represent a real nucleus. Hence, even with cutting-edge special purpose computers like GRAPE-6 (Makino 2001), $N$-body simulations can not follow the evolution of a galactic nucleus over a Hubble time if relaxation is appreciable.

The $N$ barrier can only be broken through by trading realism for efficiency. This is done mainly through three core assumptions: (1) Restricted geometry: we assume that the nucleus is of perfect spherical symmetry. (2) Dynamical equilibrium: at any given time, the system is a solution to the collisionless Boltzmann equation (Binney \& Tremaine 1987). (3) Diffusive 2-body relaxation: the departures from a smooth gravitational potential which is stationary on dynamical time scales, are treated as a large number of uncorrelated 2-body hyperbolic encounters leading to very small deflection angles. This is the base of the standard Chandrasekhar theory of relaxation (Chandrasekhar 1960).

To our knowledge, assumptions (2) and (3), which underlie the derivation of the Fokker-Planck equation from the Boltzmann equation (Binney \& Tremaine 1987), are shared by all methods aimed at simulating the relaxational evolution of stellar clusters and all of them also rely on spherical symmetry, with the exception of the code developed by Einsel \& Spurzem (1999) and Kim et al. (2002) which allows overall cluster rotation (see Paper I for a short review of these various methods). We have based our code on the Monte Carlo (MC) scheme invented by Hénon (1971b, 1971a, 1973, 1975). The reason for this choice, presented in detail in Paper I, is basically that this algorithm offers the best balance between computational efficiency, with CPU time scaling like $N_{\mathrm{p}} \ln \left(c N_{\mathrm{p}}\right)$ where $N_{\mathrm{p}}$ is the number of particles and $c$ some constant, and the ease and realism with which physics beyond relaxation, in particular stellar collisions, can be incorporated. Other codes stemming from Hénon's scheme have been developed and very successfully adapted to the dynamics of globular clusters (Stodołkiewicz 1982, 1986; Giersz 1998, 2001; Joshi et al. 2000; Watters et al. 2000; Joshi et al. 2001; Fregeau et al. 2002) but we are not aware of any previously published adaptation of this method to the realm of galactic nuclei.

In principle, there is no other restriction on the initial conditions for the cluster than conditions (1) and (2). In practice, however, the code we use to build the initial cluster (see Appendix) is limited to systems for which the distribution function (DF) depends on the energy only and doesn't account for the presence of a $\mathrm{BH}$ at the center. The first restriction implies that we cannot consider systems that present initial velocity anisotropy or mass segregation. The second forces us to start with "seed" central BHs, i.e. the BH has to be initially so light that its addition at the center of the nucleus doesn't 
noticeably perturb the dynamical equilibrium. These limitations correspond to the class of models that have been investigated in most previous studies. For instance, with the exception of Rauch (1999), all the self-consistent simulations cited above considered a seed BH which grows through accretion of stellar matter. Even though this is not a favored BH growth scenario anymore, in this paper, we adopt such models mainly as a mean to establish the correct working of our code through comparisons with the literature.

If the central $\mathrm{BH}$ forms on a time scale much shorter than relaxation time but longer than dynamical time, presumably through infall of gas from outside the nucleus, as proposed by, e.g., van der Marel (1999) and MacMillan \& Henriksen (2002), the stellar cluster reacts adiabatically, a process our code can cope with, as demonstrated in Sect. 5.1. This allows to create models at dynamical equilibrium which contains a central $\mathrm{BH}$ of significant mass. Our procedure for creating initial conditions can be adapted to clusters with central $\mathrm{BH}$ for which the energy-dependent DF is known, such as $\gamma$-models (Tremaine et al. 1994). In recent simulations to be reported in further papers, we use these models to investigate the dynamics of present-day galactic nuclei. The aim of this approach is to gain information about the rate and characteristics of interesting events (collisions, tidal disruptions, captures. .) in $z \simeq 0$ galaxies without trying to guess which are the high- $z$ "initial" conditions. However, it is observationally, as well as theoretically, doubtful that nearby galactic nuclei are devoid of anisotropy or mass segregation ${ }^{2}$. However, the lack of published generalizations of the $\gamma$-models, including mass segregation and/or anisotropy, makes it difficult to test the implications of these implicit assumptions.

The evolution of galactic nuclei is thought to go through highly dynamical phases, most noticeably mergers with other nuclei predicted by popular hierarchical structure formation scenarios. It is often assumed that the central BHs formed as intermediate mass objects $\left(100-1000 M_{\odot}\right)$ and grew mainly by luminous gas accretion during these episodes (Kauffmann \& Haehnelt 2000; Volonteri et al. 2002, and references therein) but the opposite view, i.e. that MBHs formed at high redshifts in the core of only a small fraction of proto-galaxies and grew mostly by merging together, cannot be ruled out (Menou et al. 2001). Anyway, although the MC code cannot follow these dynamical phases, one can easily use the outcome of $N$-body simulations of such processes as initial conditions, as soon as dynamical equilibrium is reached and the system is reasonably spherically symmetric. The explicit knowledge of the distribution function is not required here because one can directly turn each $N$-body particle into one or a few super-star(s).

\subsection{Units and definitions}

When we do not explicitly indicate astrophysical units, we use the "code" units defined in Sect. 3.2 of Paper I.

\footnotetext{
${ }^{2}$ For instance, we find for a model of the central cluster of the Milky Way, that significant segregation of stellar BHs appears in less than $1 \mathrm{Gyr}$ so that assuming that no segregation has occurred in the past history of the system is unrealistic.
}

$G$ is the gravitational constant. $M_{\mathrm{BH}}$ is the mass of the central $\mathrm{BH}, M_{\mathrm{cl}}$ the total stellar mass and $R_{\mathrm{cl}}$ the radius of the cluster (if finite). We use the following definition for the core radius: $R_{\mathrm{c}}=\sqrt{9 \sigma_{0}^{2} / 4 \pi G \rho_{0}}$ where $\sigma_{0}$ is the central $1 \mathrm{D}$ velocity dispersion and $\rho_{0}$ is the central density of the cluster.

We assume the following relation for the Coulomb logarithm: $\ln \left(\gamma N_{\star}\right)$ with $\gamma=0.4$ for single-mass models and $\gamma=0.01$ when there is an extended stellar mass spectrum. $N_{\star}$ is the total number of stars in the cluster. In principle, the argument of the Coulomb logarithm should be proportional to $N_{\star}$ only if the cluster is self-gravitating. In a central region of radius $G M_{\mathrm{BH}} \sigma_{\mathrm{v}}^{-2} \simeq R_{\mathrm{cl}}\left(M_{\mathrm{BH}} / M_{\mathrm{cl}}\right)$ (assuming $M_{\mathrm{BH}} \ll M_{\mathrm{cl}}, \sigma_{\mathrm{v}}$ is the velocity dispersion of the stars far from the $\mathrm{BH})$, the $\mathrm{BH}$ gravitationally out-weights the stellar cluster. There, the velocity dispersion at distance $R$ of the center is $\sigma_{\mathrm{v}}^{2}(R) \simeq G M_{\mathrm{BH}} / R$ and a steep cusp of stars is expected to develops so that, $b_{\text {max }} \simeq R$ is a sensible choice. Consequently, according to Eq. (6) of Paper I, $\Lambda \propto M_{\mathrm{BH}} / M_{\star}$ seems more appropriate (Bahcall \& Wolf 1976; Lightman \& Shapiro 1977; MiraldaEscudé \& Gould 2000). We have conducted test calculations with a $R$-variable Coulomb ratio set to $\Lambda \propto T_{\text {orb }}(R) / T_{\min }(R)$ where $T_{\text {orb }} \approx\left(G M_{r} / R^{3}\right)^{-1 / 2}$ is a measure of the orbital time and $T_{\text {min }}$ corresponds to the shortest effective 2-body encounter, i.e. $T_{\min } \approx b_{0} / \sigma_{\mathrm{v}} \approx G M_{*} \sigma_{\mathrm{v}}^{-3}$. Such a choice is motivated by the fact that a transient potential fluctuation with time scale much longer than $T_{\text {orb }}$ will act adiabatically on the motion of a given star and thus leave its orbit unchanged after it is over. Results are not significantly affected by the choice of $\Lambda$, which convinced us to keep the simple $\Lambda=\gamma N_{*}$ relation.

\section{Stellar collisions}

\subsection{Use of SPH collision simulations}

The inclusion of realistic collisions ${ }^{3}$ is probably the main improvement over previous cluster evolution codes that our scheme features. In the past few years, we have been computing thousands of 3D hydrodynamics simulations of collisions between MS stars using a SPH code (Benz 1990). For simplicity, only collisions between main sequence stars are considered for the time being. Actually, giant stars are expected to dominate the collision rate (Bailey \& Davies 1999; Freitag \& Benz 2002a). The effects of collisions are included in the cluster simulations with unpreceded realism by interpolating the outcome of these events from the huge SPH-generated results database (Freitag \& Benz 2002a). In so doing, we get rid of many of the uncertainties introduced by the simplistic recipes formerly used in simulations of collisional cluster dynamics. Unfortunately, even with such a procedure, important simplifications of the physics have still to be done. The major ones are connected with the possible formation of binaries through tidal dissipation of orbital energy and to the stellar evolution of the stars that have suffered from a collision. We discuss both problems in turn.

\footnotetext{
3 Here, by "collision", we mean a genuine hydrodynamical contact encounter between two stars, as opposed to mere 2-body gravitational deflections.
} 
The cross sections for the formation of so-called "tidalbinaries" are not well known (Press \& Teukolsky 1977; Lee \& Ostriker 1986; McMillan et al. 1987; Benz \& Hills 1992; Lai et al. 1993; Kim \& Lee 1999) and their long-term evolution is still debated (Benz \& Hills 1992; Lai et al. 1993). Hence, it is fortunate that the rate of tidal captures is overtaken by the rate of collisions as soon as $\sigma_{\mathrm{v}} / v_{*}>0.1$ where $\sigma_{\mathrm{v}}$ is the $1 \mathrm{D}$ velocity dispersion of the stars and $v_{*}=\sqrt{2 G M_{*} / R_{*}}$ is the escape velocity at the surface of a star (Lai et al. 1993, Fig. 16). Consequently, as we expect quite high stellar velocities in the center of galactic nuclei (particularly near a supermassive $\mathrm{BH}$ ), we decided to neglect tidal capture in our code.

A parameter of prime importance is the star's radius as it determines its collisional cross section and, hence, the probability of subsequent collisions that could lead, for instance, to the runaway build-up of more and more massive stars by multiple mergers. After a collision, as a large amount of energy has been injected into the stellar envelope, the star is much larger than a MS star with the same mass. However, on a Kelvin-Helmholtz time scale $\left(T_{\mathrm{KH}}\right)$ the radius shrinks back to the MS value, as the stellar structure returns to thermal equilibrium. Here we assume $T_{\text {coll }} \gg T_{\mathrm{KH}}$ so we can neglect the short swollen phase and attribute a MS radius to the collision product.

When stellar evolution is taken into account, it becomes in principle necessary to know what amount of collisional mixing occurs and how it affects the MS life-time $T_{\mathrm{MS}}$ of the product. We can expect that, contrary to parabolic mergers where only little mixing takes place (Sills et al. 1997, for instance), high velocity collisions are able to rejuvenate the star by bringing fresh hydrogen-rich gas from the outer parts to the center. If two stars of unequal masses merge together, simulations show that the smaller one, whose material is of lower entropy, sinks to the center of the larger one (Lombardi et al. 2002). This appears as an efficient mechanism to bring fuel directly to the core of the large star and delay hydrogen exhaustion. Conversely, the higher mean molecular weight $\mu$ that results from spreading the central Helium (produced by H-burning on the MS) leads to a important decrease of $T_{\mathrm{MS}}$ as compared to a star with a "normal" composition (Claret \& Gimenez 1998). Indeed, from homological relations, one finds: $T_{\mathrm{MS}} \propto \mu^{-4}$ (Kippenhahn \& Weigert 1994). On the other hand, the radius depends only weakly on $\mu$ ( $R \propto \mu^{0.6}$ for the CNO-cycle) so we can safely neglect the effects on the collision cross section in our simulations ${ }^{4}$.

\subsection{Collision rate}

Let's consider a close approach between two stars with masses and radii $M_{1}, R_{1}$ and $M_{2}, R_{2}$, respectively. The relative velocity at infinity is $v_{\text {rel }}$ and the impact parameter $b$. A collision occurs when the centers of the stars are closer to each other than $d=\eta\left(R_{1}+R_{2}\right)(\eta=1$ for genuine collision, $\eta \leq 1$ for merging, $\eta \geq 1$ for tidal capture when $v_{\text {rel }}$ is small enough). Until this

\footnotetext{
4 How the outcome of further collisions will be influenced by structural changes due to previous collisions has not yet been assessed. This can be of importance in the case of "run-away" mergers.
}

collision distance is reached, we neglect the gravitational influence of other stars as well as any mutual tidal interaction. So the problem reduces to a simple hyperbolic approach between two point masses. This gives us, the largest impact parameter leading to contact, $b_{\max }$, and the cross section,

$$
S_{\text {coll }}^{(12)}=\pi b_{\max }^{2}=\pi \eta^{2}\left(R_{1}+R_{2}\right)^{2}\left(1+\frac{\left(v_{*}^{(12)}\right)^{2}}{\eta v_{\text {rel }}^{2}}\right)
$$

where

$v_{*}^{(12)}=\sqrt{\frac{2 G\left(M_{1}+M_{2}\right)}{R_{1}+R_{2}}}$.

The second term is the bracket of equation 1 is the gravitational focusing which enhances the cross-section at low velocity $\left(S_{\text {coll }}^{(12)} \propto R_{1}+R_{2}\right)$. At high velocities $S_{\text {coll }}$ tends to the geometrical value $\pi f^{2}\left(R_{1}+R_{2}\right)^{2}$. So, the collision rate for a test-star " 1 " in a field of stars " 2 " having all the same mass, radius and relative velocity to " 1 " is simply

$$
\left.\frac{\mathrm{d} N_{\text {coll }}}{\mathrm{d} t}\right|^{(1 ; 2)}=n_{2} v_{\mathrm{rel}} S_{\mathrm{coll}}^{(12)}
$$

where $n_{2}$ is the (local) number density of stars " 2 ".

If we are interested in the overall collision rate in a star cluster, the next step to do is to introduce a velocity distribution. Before considering more general cases, let's assume that all stars in the cluster have the same mass $M_{*}$ and radius $R_{*}$ (so we can drop over-score "(12)" in $v_{*}$ and $S_{\text {coll }}$ ) and that their density is $n_{*}$. The average local collision time $T_{\text {coll }}(R)$ is found by integrating the collision rate (Eq. (3)) over all possible velocities of the two stars,

$$
\frac{1}{T_{\text {coll }}}=\frac{1}{n_{*}} \int \mathrm{d}^{3} \boldsymbol{v}_{1} \mathrm{~d}^{3} \boldsymbol{v}_{2} f\left(\boldsymbol{v}_{1}\right) f\left(\boldsymbol{v}_{2}\right)\left\|\boldsymbol{v}_{1}-\boldsymbol{v}_{2}\right\| S_{\text {coll }} .
$$

As shown in Binney \& Tremaine (1987), the result for a Maxwellian distribution is

$$
\left.\frac{1}{T_{\text {coll }}}\right|_{\mathrm{M}}=\underbrace{16 \sqrt{\pi}}_{\simeq 28.4} \eta^{2} R_{*}^{2} n_{*} \sigma_{\mathrm{v}}\left(1+\frac{v_{*}^{2}}{4 \eta \sigma_{\mathrm{v}}^{2}}\right) .
$$

For the Plummer model, the result is very similar to Eq. (5), with only the numerical constant replaced by 28.6 .

The total number of collisions per unit time in the cluster is given by the integration of $1 / T_{\text {coll }}(R)$ over the whole cluster:

$$
\left.\frac{\mathrm{d} N_{\text {coll }}}{\mathrm{d} t}\right|_{\text {tot }}=\frac{1}{2} \frac{N_{*}}{\widehat{T}_{\text {coll }}}=2 \pi \int_{0}^{\infty} \mathrm{d} R R^{2} n_{*}(R) \frac{1}{T_{\text {coll }}(R)} .
$$

For a Plummer model of total mass $M$, star number $N_{*}$ and scale radius $R_{\mathrm{P}}$, the collision rate by unit radius reads:

$$
\begin{aligned}
\frac{\mathrm{d} N_{\text {coll }}}{\mathrm{d} t \mathrm{~d} R}(R)= & 54 \sqrt{2} \frac{\sqrt{G \rho_{0}}}{R_{\mathrm{P}}} \frac{1}{\Theta_{0}^{2}} \\
& \times u^{2}\left(1+u^{2}\right)^{-21 / 4}\left[1+\Theta_{0}\left(1+u^{2}\right)^{1 / 2}\right]
\end{aligned}
$$

with

$u=\frac{R}{R_{\mathrm{P}}}, \quad \rho_{0}=\rho(0)=\frac{3}{4 \pi} \frac{M}{R_{\mathrm{P}}^{3}}$, 


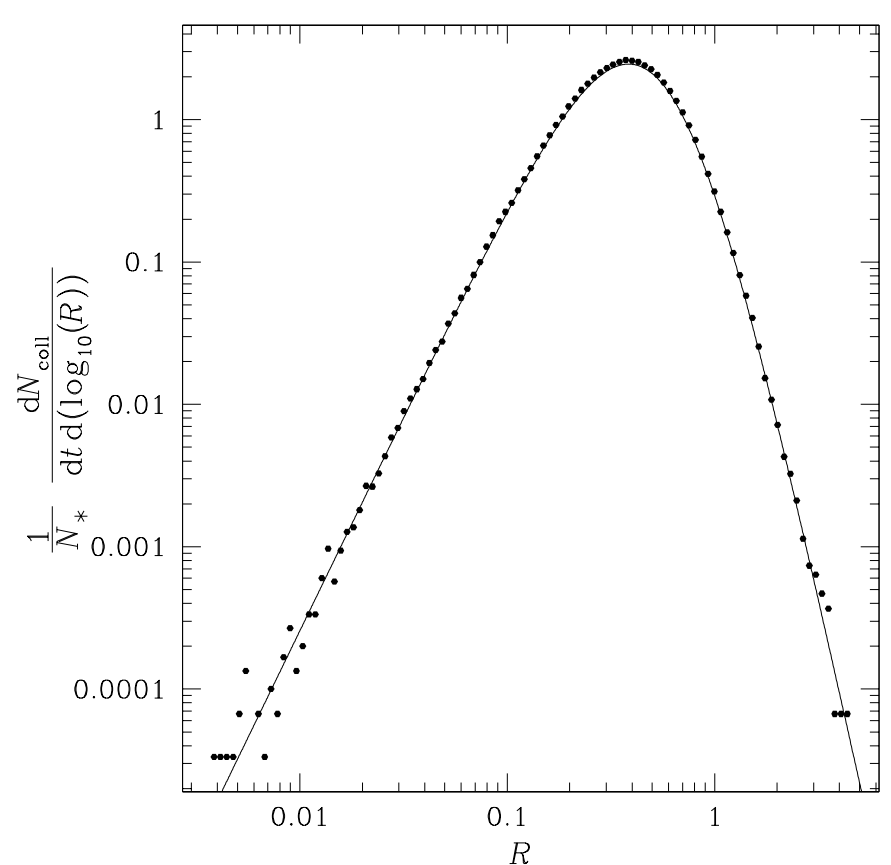

Fig. 1. Collision rate as a function of radius in a Plummer cluster with $\Theta_{0}=0.725$ and $N_{*}=10^{6}$. The solid line is the theoretical rate based on Eq. (7). The dots are statistics from a MC simulation run with no cluster evolution. " $N$-body units", $\mathcal{U}_{1}$ and $\tilde{\mathcal{U}}_{\mathrm{t}}$ are used (see Sect. 1.3).

and

$\Theta_{0}=\frac{v_{*}^{2}}{4 \eta \sigma_{\mathrm{v}}^{2}(0)}=\frac{3}{\eta N_{*}} \frac{R_{\mathrm{P}}}{R_{*}}$ (Safronov number).

As a check of our code, Fig. 1 depicts this rate along with the statistics produced in a inventory run during which the cluster's structure as a Plummer model was frozen.

Carrying out the radial integration, we finally get the total collision rate in the whole Plummer cluster:

$$
\left.\frac{\mathrm{d} N_{\text {coll }}}{\mathrm{d} t}\right|_{\text {tot }}=\sqrt{G \rho_{0}} \frac{1}{\Theta_{0}^{2}}\left(4.25+5.20 \Theta_{0}\right) \text {. }
$$

\subsection{Relative collision rates between stars of different masses}

We now address the case of a cluster with a distribution of stellar masses. For simplicity, we consider a discrete mass spectrum with $N_{\mathrm{sp}}$ components: $M_{*} \in\left\{M_{i}\right\}_{i=1}^{N_{\mathrm{sp}}}$ with (local) densities $n_{i}$. So, using Eq. (3), the rate by unit volume for collisions between stars of classes $i$ and $j$, with velocities $\boldsymbol{v}_{i}$ and $\boldsymbol{v}_{j}$ is

$\Gamma_{i j} \mathrm{~d}^{3} \boldsymbol{v}_{i} \mathrm{~d}^{3} \boldsymbol{v}_{j}=f_{i}\left(\boldsymbol{v}_{i}\right) f_{j}\left(\boldsymbol{v}_{j}\right)\left\|\boldsymbol{v}_{i}-\boldsymbol{v}_{j}\right\| S_{\text {coll }}^{(i j)} \mathrm{d}^{3} \boldsymbol{v}_{i} \mathrm{~d}^{3} \boldsymbol{v}_{j}$

where $f_{i}, f_{j}$ are the phase-space DFs which are assumed to comply with (spatial) spherical symmetry and isotropy. Their $R$-dependence is implicit. If we further assume Maxwellian velocity distributions with 1-D velocity dispersions $\sigma_{i}$ and $\sigma_{j}$, the distribution of the relative velocity $\boldsymbol{v}_{\text {rel }}=\boldsymbol{v}_{i}-\boldsymbol{v}_{j}$ is Maxwellian too, with dispersion $\sigma_{i j}=\sqrt{\sigma_{i}^{2}+\sigma_{j}^{2}}$. We keep $v_{\text {rel }}=\left\|\boldsymbol{v}_{\text {rel }}\right\|$ as the only relevant velocity variable by integrating Eq. (9) over the others:

$\Gamma_{i j} \mathrm{~d} v_{\text {rel }} \propto n_{i} n_{j}\left(R_{i}+R_{j}\right)^{2} v_{\text {rel }}^{3}\left(1+\left(\frac{v_{*}^{(i j)}}{v_{\text {rel }}}\right)^{2}\right) \sigma_{i j}^{-3} \mathrm{e}^{-\frac{1}{2} \frac{v_{\text {rel }}^{2}}{\sigma_{i j}^{2}}} \mathrm{~d} v_{\text {rel }}$.

$\eta$ has been set to 1 . For a continuous mass spectrum, we define the mass function as $\psi\left(M_{i}\right)=n_{*}^{-1} \mathrm{~d} n_{*}\left(M_{i}\right) / \mathrm{d}\left(\log _{10}\left(M_{i}\right)\right)$ so we have to substitute $\mathrm{d} n_{i}=n_{*} \psi\left(M_{i}\right) \mathrm{d}\left(\log _{10}\left(M_{i}\right)\right)$ for $n_{i}$ in the previous formula. In order to get an equation for the relative collision rate between stars of different masses (per unit volume, $\log _{10}\left(M_{i}\right)$ and $\log _{10}\left(M_{j}\right)$ ), we assume $\sigma_{i}=\sigma_{j}=\sigma_{\mathrm{v}} \forall i, j$ and integrate over $v_{\text {rel }}$ :

$\Gamma\left(M_{i}, M_{j}\right) \propto n_{*}^{2} \sigma_{\mathrm{v}} \psi\left(M_{i}\right) \psi\left(M_{j}\right)\left(R_{i}+R_{j}\right)^{2}\left(1+\Theta^{(i j)}\right)$

with $\Theta^{(i j)}=\frac{\left(v_{*}^{(i j)}\right)^{2}}{4 \sigma_{\mathrm{v}}^{2}}=\frac{G\left(M_{i}+M_{j}\right)}{2 \sigma_{\mathrm{v}}^{2}\left(R_{i}+R_{j}\right)}$.

For a Plummer model with no mass-segregation (and, thus, a unique $\sigma_{\mathrm{v}}(R)$ ), this relation, when integrated over the whole cluster, leads to

$$
\begin{aligned}
\Gamma_{\text {tot }}\left(M_{i}, M_{j}\right)= & \frac{\mathrm{d} N_{\text {coll }}}{\mathrm{d} t \mathrm{~d}\left(\log _{10} M_{i}\right) \mathrm{d}\left(\log _{10} M_{j}\right)} \\
\propto & \psi\left(M_{i}\right) \psi\left(M_{j}\right) \sqrt{G \rho_{0}} N_{*}^{2}\left(\frac{R_{i}+R_{j}}{R_{\mathrm{P}}}\right)^{2} \\
& \times\left(1+3.66 \frac{M_{i}+M_{j}}{M} \frac{R_{\mathrm{P}}}{R_{i}+R_{j}}\right) \\
\propto & \psi\left(M_{i}\right) \psi\left(M_{j}\right)\left(\frac{R_{i}+R_{j}}{R_{\odot}}\right)^{2} \\
& \times\left(1+\widehat{\Theta} \frac{\left(M_{i}+M_{j}\right) / M_{\odot}}{\left(R_{i}+R_{j}\right) / R_{\odot}}\right)
\end{aligned}
$$

with $\widehat{\Theta}=3.66 \frac{R_{\mathrm{P}} / R_{\odot}}{M / M_{\odot}}$.

In relation (13), only the dependencies on stellar quantities have been preserved to insist on the relative collision rates between different stellar species. Although it relies on the admittedly unrealistic hypothesis of no mass segregation, Eq. (13) proves useful as a prediction our code can easily (and successfully) be tested against, see Fig. 2 .

\subsection{Introduction of stellar collisions in the MC code}

The difficulty of introducing stellar collisions in any stellar dynamics code is twofold. First, as the previous discussion has shown, it is not at all straightforward to determine the correct distribution of collision parameters $\left(v_{\text {rel }}\right.$, star types, position in the cluster, ... ). Secondly, provided the result of a particular collision is known (by performing hydrodynamical simulations, for instance), we want to be able to preserve as much as possible of that valuable information when introducing it back in the cluster evolution code. Due to their very structure ${ }^{5}$,

5 Their basic limitation lies in the principle they owe their efficiency to: they model the stellar system as a set of continuous DFs (one for each different stellar mass). 
a)

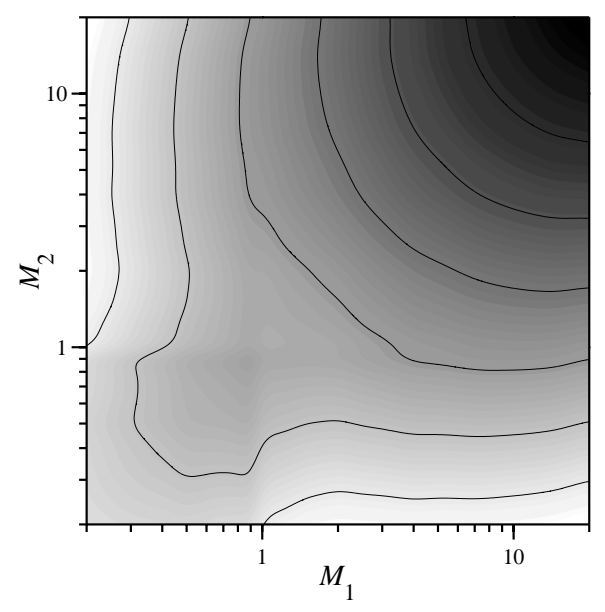

b)

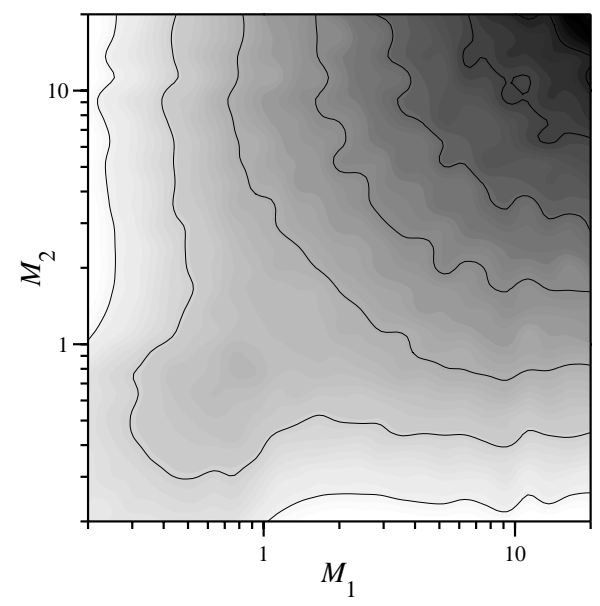

Fig. 2. Total relative collision rate $\Gamma_{\text {tot }}\left(M_{1}, M_{2}\right)$ between stars with masses $M_{1}$ and $M_{2}$ in a Plummer cluster without mass-segregation. The gravitational focusing parameter is $\widehat{\Theta}=1.5$. Masses are in $M_{\odot}$. Lighter gray shades correspond to higher values. Successive contour levels correspond to factor of 2 decrease in $\Gamma$. The same levels are drawn on both panels. The mass-function is $\Psi\left(M_{*}\right) \propto M_{*}^{-1.35}$ for $M_{*}$ between 0.2 and $20 M_{\odot}$ and the Mass-Radius relation is set according to stellar models by Schaller et al. (1992) and Charbonnel et al. (1999). a) Theoretical rate from Eq. (13). b) Statistics from the MC code $(400000$ collisions) with cluster evolution inhibited. This comparison demonstrates the accuracy of the collision sampling in our code. some widely used schemes, based on an explicit resolution of the Fokker-Planck equation, impose such a highly simplified treatment of the collisions' outcome that it would not make much sense to devote energy to a realistic computation of these events. The MC method is exempt of such limitations.

\subsubsection{Global code modifications}

Collisions introduce a new time scale in the code. There is consequently a new constraint on the time steps

$\delta t(R) \leq f_{\delta t} \tilde{T}_{\text {coll }}(R)$.

$\tilde{T}_{\text {coll }}(R)$ is an estimation of the local collision time. We chose the following definition, based on Eq. (5):

$\frac{1}{\tilde{T}_{\text {coll }}(R)}=16 \sqrt{\pi} n_{*} \sigma_{\mathrm{v}}\left\langle R_{*}^{2}\right\rangle\left[1+\frac{G\left\langle M_{*} R_{*}\right\rangle}{2 \sigma_{\mathrm{v}}^{2}\left\langle R_{*}^{2}\right\rangle}\right]$

where $\sigma_{\mathrm{v}}^{2}=\left\langle v^{2}\right\rangle$ and $\langle$ bracketed $\rangle$ quantities are local averages. This particular expression was chosen for its ease of evaluation and because, provided all stellar species have isothermal velocity distribution (quite a strong demand!), it reduces to exact relations in the two interesting limiting cases:

$\tilde{T}_{\text {coll }}^{-1} \rightarrow\left\langle T_{\text {coll }}^{-1}\right\rangle= \begin{cases}8 \sqrt{\pi} G n_{*} \frac{\left\langle M_{*} R_{*}\right\rangle}{\sigma_{\mathrm{v}}} & \text { for } \sigma_{\mathrm{v}}^{2} \ll\left\langle v_{*}^{2}\right\rangle \\ 16 \sqrt{\pi} n_{*} \sigma_{\mathrm{v}}\left\langle R_{*}^{2}\right\rangle & \text { for } \sigma_{\mathrm{v}}^{2} \gg\left\langle v_{*}^{2}\right\rangle .\end{cases}$

By requiring

$\delta t(R) \leq f_{\delta t}\left(\tilde{T}_{\text {rel }}^{-1}(R)+\tilde{T}_{\text {coll }}^{-1}(R)\right)^{-1}$,

we make sure that time steps are short enough to resolve both relaxational and collisional processes. Apart for this extended constraint, all the time-step determination and pair selecting machinery of Paper I is left formally unchanged.

\subsubsection{Monte Carlo sampling of the collisions}

Relaxation is due to the cumulative effects of a huge number a small individual scatterings and can be treated as a continuous process, affecting progressively the particles' orbits. To be computationally tractable this phenomenon is discretized back into "super-encounters". In contrast, collisions do not act gradually but are genuinely discrete events, each of which strongly affect the properties of the implied stars. Hence, there seems to be no way to add up the effects of collisions into "supercollisions", no escape from the necessity to simulate them as individual events.

When a pair of adjacent super-stars is selected to be evolved for a time step $\delta t$, we randomly orient their velocities and compute the local number density of stars of any kind, $n_{*}$, as explained in Paper I. The probability for a mutual collision to occur during that time span is, adapting Eq. (3),

$P_{\text {coll }}^{(12)}=N_{\text {coll }}^{(12)}=n_{*} v_{\text {rel }} S_{\text {coll }}^{(12)} \delta t$.

When compared to Eq. (3), this expression could be thought to be an overestimate as $n_{*}$ is used instead of $n_{2}$. Actually, for a given super-star of type "1", the expectation value for the number of collisions with super-stars of type " 2 " is

$$
\begin{aligned}
& \left\langle N_{\text {coll }}^{(12)}\right\rangle=\underbrace{\left[\begin{array}{l}
\text { Prob. for neigh- } \\
\text { bor of being of } \\
\text { type 2 }
\end{array}\right]}_{n_{2} / n_{*}} \cdot \underbrace{\left[\begin{array}{l}
\text { Collision prob. if } \\
\text { neighbor is of } \\
\text { type 2 }
\end{array}\right]}_{P_{\text {coll }}^{(12)}} \\
& =n_{2} v_{\mathrm{rel}} S_{\text {coll }}^{(12)} \delta t \text {, }
\end{aligned}
$$

as needed. The collision probability is compared with a random number $X_{\text {rand }}$ with [0;1[-uniform deviate. If $X_{\text {rand }}<P_{\text {coll }}^{(12)}$, a collision has to be simulated whose initial conditions are completely determined as soon as a value of the impact parameter $b$ has been chosen according to probability density

$\mathrm{d} P= \begin{cases}\frac{2 b \mathrm{~d} b}{b_{\max }^{2}} & \text { if } 0 \leq b \leq b_{\max }, \\ 0 & \text { otherwise. }\end{cases}$ 
Note that the super-star pair is tested for collisions before relaxation is applied to it. In case a collision is suffered, the orbits are probably deeply modified. So the relaxation step is skipped even if the pair survived.

\subsubsection{Treatment of an individual collision}

As explained earlier, the outcome of collisions happening in the course of the cluster's evolution is specified by a large set of 3D hydrodynamical simulations. These are potentially able to provide us with any detail, significant or not, about the state of the resulting star(s) and released gas. Most of this information, however is of no real relevance so we focus on the important parameters we have to sort out of this data and plug into the MC code. In another paper (Freitag \& Benz 2002a), we will describe the way collisions are simulated with an SPH code and how we extract the needed "macroscopic" information back from the simulation. Suffice to say that, if we assume the center of mass $(\mathrm{CM})$ reference frames defined before and after the collision are the same (i.e. that $M_{1}^{\prime} w_{1}^{\prime}+M_{2}^{\prime} w_{2}^{\prime}=0$ where $M_{1,2}^{\prime}$ and $\boldsymbol{w}_{1,2}^{\prime}$ are the post-collision masses and velocity vectors in the pre-collision CM frame), the kinematic outcome is entirely described by 4 numbers. They are $M_{1}^{\prime}, M_{2}^{\prime}$, the final relative velocity at infinity,

$v_{\text {rel }}^{\prime}=\sqrt{\frac{2 E_{\text {orb }}^{\prime}}{M_{1}^{\prime} M_{2}^{\prime} /\left(M_{1}^{\prime}+M_{2}^{\prime}\right)}}$

and the deflection angle $\theta_{\text {coll }}$. Further information is contained in the post-collision stellar structure but it may be ignored if one assumes, as we do, that the produced star(s) return to normal MS structure. These 4 numbers are all we need to implement collisions between super-stars following exactly the same scheme as described in Sect. 4.2.1 of Paper I (steps 2-4) for purely gravitational encounters. The only added difficulty is connected with mass changes and the proper tracking of energy variation they imply.

Note that when a collision between two super-stars occurs, it amounts to each star in the first super-star colliding with a star from the second super-star. As the number of stars per superstar is the same by construction, one can apply the outcome of the collision (new mass and velocity) uniformly to all stars of the super-star, i.e. to the super-star as a whole. When the stellar collision results in two surviving stars, we have to modify the orbital and stellar properties of both super-stars; when there is only one star left (merger or destruction of the smaller star, see Freitag \& Benz 2002b), one superstar is removed and the other one is given the properties of the remaining star; if both stars are destroyed, both super-stars are removed from the simulation.

\section{Tidal disruptions}

\subsection{Loss cone theory}

If a star ventures very close to the $\mathrm{BH}$, it may be broken apart by tidal forces. The condition for an element of mass to be stripped away from the surface of the star is that the instantaneous gravitational attraction on it (due to the $\mathrm{BH}$ and the star itself) be lower than the required centripetal acceleration. In the simplified case of a non-rotating ${ }^{6}$ spherical star on a Keplerian orbit, this condition determines the following disruption radius:

$R_{\mathrm{disr}} \simeq\left(2 \frac{M_{\mathrm{BH}}}{M_{*}}\right)^{1 / 3} R_{*}=\left(\frac{3}{2 \pi} \frac{M_{\mathrm{BH}}}{\rho_{*}}\right)^{1 / 3}$.

Where $\rho_{*}$ is the average density of the stellar matter. This approximation assumes $M_{\mathrm{BH}} \gg M_{*}$. Note that this is really only the condition for the tidal stripping of the outer layers of gas because the stellar density increases toward the center of the star. A more realistic approach should account for elliptical or parabolic orbits, tidally induced deformation and the genuine hydrodynamical nature of this violent phenomenon. Moreover, if deep encounter certainly result in complete star destruction, milder ones would be responsible of partial envelope stripping. Many studies have addressed these aspects (Carter \& Luminet 1983; Evans \& Kochanek 1989; Laguna et al. 1993; Fulbright 1996; Ayal et al. 2000). Fulbright performed SPH simulations of parabolic encounters whose strength can be parameterized by

$\beta=\frac{R_{*}}{R_{\text {peri }}}\left(\frac{M_{\mathrm{BH}}}{M_{*}}\right)^{1 / 3}$.

For polytropic star models with $n=3 / 2$ and $n=3$, he found that stripping of half the stellar mass occurs for $\beta_{\mathrm{h}} \simeq 0.8$ and $\beta_{\mathrm{h}} \simeq 1.7$, respectively. In the present version of our code, complete disruption is assumed for $\beta>\beta_{\mathrm{h}}$ while the star is left undamaged for more distant encounters. This corresponds to Eq. (21) with the factor $2^{1 / 3}$ replaced by $\beta_{\mathrm{h}}^{-1}$.

The "loss orbits" are the set of stellar orbits with pericenter distance $R_{\text {peri }}$ smaller than $R_{\text {disr }}$. For a star at distance $R$ to the center with velocity modulus $v$, the loss cone (LC) is the set of velocity directions that leads $R_{\mathrm{a}}<R_{\mathrm{disr}}$, either going to the $\mathrm{BH}$ our coming from it (see Fig. 3). The aperture angle of the loss-cone, $\theta_{\mathrm{LC}}$, is given by the relation

$$
\begin{aligned}
\sin ^{2}\left(\theta_{\mathrm{LC}}\right)= & 2\left(\frac{R_{\mathrm{disr}}}{v R}\right)^{2}\left[\frac{v^{2}}{2}+\frac{G M_{\mathrm{BH}}}{R_{\mathrm{disr}}}\left(1-\frac{R_{\mathrm{disr}}}{R}\right)\right. \\
& \left.+\Phi_{*}(R)-\Phi_{*}\left(R_{\mathrm{disr}}\right)\right]
\end{aligned}
$$

where $\Phi_{*}(R)=\Phi(R)+G M_{\mathrm{BH}} / R$ is the cluster contribution to the gravitational potential. As, for reasonable parameters, $R_{\mathrm{disr}}$ is a tiny value, typical loss orbits are very elongated, so that $R \gg R_{\text {disr }}$ and $G M_{\mathrm{BH}} / R_{\mathrm{disr}} \simeq v_{*}^{2}\left(M_{\mathrm{BH}} / M_{*}\right)^{2 / 3} \gg v^{2}$. Hence Eq. (23) simplifies to

$$
\theta_{\mathrm{LC}}^{2} \simeq 2 \frac{G M_{\mathrm{BH}} R_{\mathrm{disr}}}{v^{2} R^{2}}
$$

The loss cone is usually very small, as is demonstrated by an order-of-magnitude estimate of $\theta_{\mathrm{LC}}$ at the $\mathrm{BH}$ 's "influence

\footnotetext{
6 In case of a co-rotating spherical star on a circular orbit, one gets a factor 3 instead of 2 inside $(\cdots)^{1 / 3}$.
} 


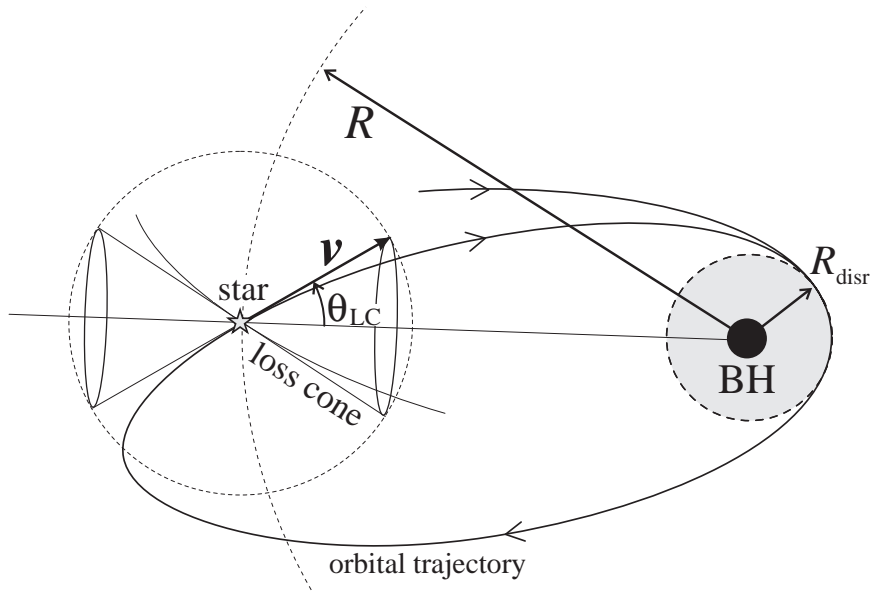

Fig. 3. Diagram of the loss cone.

radius" $\left(R_{\mathrm{i}}=G M_{\mathrm{BH}} / \sigma_{\mathrm{v}}^{2}\right)$ :

$$
\begin{aligned}
\theta_{\mathrm{LC}}^{2}\left(R_{\mathrm{i}}\right) \simeq & N_{*}\left(\frac{M_{*}}{M_{\mathrm{BH}}}\right)^{2 / 3} \frac{R_{*}}{R_{\mathrm{h}}} \\
\simeq & 2 \times 10^{-5}\left(\frac{N_{*}}{10^{7}}\right)\left(\frac{M_{\mathrm{BH}}}{10^{6} M_{\odot}}\right)^{-\frac{2}{3}}\left(\frac{R_{\mathrm{h}}}{1 \mathrm{pc}}\right)^{-1} \\
& \text { for } R_{*}=1 R_{\odot} \text { and } M_{*}=1 M_{\odot} .
\end{aligned}
$$

$R_{\mathrm{h}}$ is the cluster's half-mass radius.

If it wasn't for relaxation or other orbit modifying mechanisms (collisions for instance) these loss orbits, if initially populated, would be drained over a dynamical time and no further tidal disruption would be expected in the subsequent cluster evolution, unless some increase of $R_{\text {disr }}$ occurs. This could happen for the whole cluster as a result of the $\mathrm{BH}$ accreting gas supplied to it by other sources like stellar winds $\left(M_{\mathrm{BH}} \nearrow\right)$, or, as investigated by Syer \& Ulmer (1999), for those stars that experience rapid swelling when they become red giants $\left(R_{*} \nearrow\right)$.

The crux of determining the rate of tidal disruptions, however, is the role of relaxation. This process is capable of replenishing loss cone orbits while at the same time it can remove stars from such orbits thus preventing them from being disrupted. These effects have been tackled either using quite rigorous approaches (Lightman \& Shapiro 1977; Cohn \& Kulsrud 1978; Magorrian \& Tremaine 1999) mainly aimed at their inclusion into Fokker-Planck codes, or resorting to more approximate descriptions (Frank \& Rees 1976; Syer \& Ulmer 1999; Miralda-Escudé \& Gould 2000). Here we only outline the problem by recalling a few simple facts.

Equation (24) can be recast in a simple characterization of loss orbits:

$$
J^{2} \leq J_{\mathrm{LC}}^{2} \simeq 2 G M_{\mathrm{BH}} R_{\mathrm{disr}},
$$

a condition independent of energy $E$ (for stars not too tightly bound to the $\mathrm{BH}$ ). Thus the flux of stars to/from disruption orbits is chiefly controlled by $J$-"diffusion" in the vicinity of the $J_{\mathrm{LC}}$ borderline. For a given star, let $\delta J_{\text {orb }}$ be the mean quadratic variation of the angular momentum due to relaxation during a single orbit (defined as the trajectory segment from a passage to apocenter position to the next one),

$\delta J_{\mathrm{orb}}=\sqrt{\left\langle(\Delta J)^{2}\right\rangle_{P_{\mathrm{orb}}}}$.

If $\delta J_{\text {orb }} \gg J_{\mathrm{LC}}$, stars can survive many orbits, scattered into and out of loss trajectories before being tidally disrupted. It follows that orbits with $J<J_{\mathrm{LC}}$ are not strongly depleted and this regime is referred to as full loss cone. If the velocity distribution is initially isotropic, this process doesn't modify that fact and the fraction of stars disrupted per orbital period is simply those of velocity directions pointing in the loss-cone:

$\frac{\mathrm{d} \dot{N}_{\text {full }}}{\mathrm{d} N}=\frac{1}{P_{\text {orb }}} \frac{\theta_{\mathrm{LC}}^{2}}{2}$.

Conversely, in the empty loss cone limit, $\delta J_{\text {orb }} \ll J_{\mathrm{LC}}$, there is no way back from the loss orbits and the situation can be described as a genuine diffusion in $J$-space. At a given energy, the star density in $J$-space gradually goes to zero as $J_{\mathrm{LC}}$ is approached from above. This negative gradient controls the diffusive flux of stars to the lethal loss orbits. Analytical treatment of this regime is far beyond the scope of this paper so we refer the interested reader to the above-mentioned previous studies and turn to a description of our MC approach to the problem.

\subsection{Implementation of loss cone effects}

A reliable determination of the tidal disruption rate requires for the numerical simulation of the relaxation process a resolution $\delta J_{\text {num }}<J_{\mathrm{LC}}$ in the empty loss-cone regime and $\delta J_{\text {num }}=\delta J_{\text {orb }}$ in the full loss-cone regime. The latter case could be treated by use of Eq. (29) as a quick shortcut but the former constraint cannot be circumvented as easily. Unfortunately, whereas simulation of "normal" relaxation imposes a value of the numerical deviation angle per step, $\delta \theta_{\text {step }}$ sufficiently smaller than $\pi$ $\left(\delta \theta_{\text {step }} \simeq \pi / 2 \sqrt{f_{\delta t}} \simeq 0.1 \pi\right.$, see Eqs. (7) and of (10) Paper I), resolution of the (empty) loss cone region is not attained unless $\delta \theta_{\text {step }}<\theta_{\mathrm{LC}} \ll \pi$ ! Furthermore, a foolproof approach, not relying on a clear-cut a priori distinction between "full" and "empty" regimes, would necessitate to reduce $\delta \theta_{\text {step }}$ to the tiny "elementary" orbital $\delta \theta_{\text {orb }}$ step with a corresponding $\delta t_{\text {step }}=P_{\text {orb }} \simeq \ln \left(\gamma N_{*}\right) N_{*}^{-1} T_{\text {rel }}$, thousands of times smaller than the desired $\delta t_{\text {step }} \simeq f_{\delta t} T_{\text {rel }}$ ! Although Shapiro (1985) was able to attribute such tiny $\delta t$ only to those particles orbiting close to (or inside) the LC, hence preventing too drastic a code slowing down, such a feature doesn't fit in any straightforward way into Hénon's scheme. To mention but one impediment, the need of devising time steps that depend only on the super-star's radial rank would impose $\delta t \simeq P_{\text {orb }}$ for a large fraction of super-stars.

The simple structure of our code - mainly consisting in successive 2-super-star interaction steps - having proved to be both easy to grasp conceptually and reliable when applied to relaxational and collisional simulations, we introduced loss cone effects in a way that required the least modifications.

Let's consider a single step. If the encounter was a collision, we only need to test whether each surviving super-star entered the LC through the interaction and to disrupt it in such a 
case. Indeed, collisions are not to be refined into more elementary processes. On the other hand, after a gravitational superencounter has been computed, with deflection angle $\delta \theta_{\text {step }}$ in the encounter reference frame, each surviving super-star is examined for tidal disruption in turn by simulating its random walk (RW) in $J$-space during $\delta t_{\text {step }}$. In MC spirit, we estimate typical "representative" for the diffusion angle during a single orbit, $\delta \theta_{\text {orb }}$ by scaling down $\delta \theta_{\text {step }}$ to orbital time,

$\delta \theta_{\text {orb }} \equiv n_{\text {orb }}^{-1 / 2} \delta \theta_{\text {step }} \quad$ with $n_{\text {orb }}=\frac{\delta t_{\text {step }}}{P_{\text {orb }}}$.

Let $\boldsymbol{w}$ be the super-star's velocity vector in the encounter frame. We decompose the step $\delta t_{\text {step }}$ into a random walk of the tip of $\boldsymbol{w}$ on a sphere with fixed $w=\|\boldsymbol{w}\|$ radius, starting at its initial direction. A brute force implementation would require up to $n_{\text {orb }}$ steps of angular size $\delta \theta_{\text {orb }}$, each one followed by a test for entry into the LC (Eq. (27)). The number of orbits per $\delta t_{\text {step }}$ typically ranging from $10^{3}$ to $10^{6}$, such a procedure turns out to be extremely inefficient, requiring a huge number of operations to detect only a few tidal disruptions, even if super-stars with initial velocities pointing too far from the LC are filtered out ${ }^{7}$. Fortunately, the burden can be lighten enormously through use of adaptive RW steps. Indeed, $n$ individual steps of length $\delta$ with random relative orientation are statistically nearly equivalent to a single "meta" one of length $\Delta=\sqrt{n} \delta$, ${ }^{8}$ as long as $\Delta$ is sufficiently smaller than the distance to the LC, to keep the risk of missing a disruption during these $n$ RW steps at very low level. Here is the outline of the random walk procedure:

1. Preparation. The orbital period is integrated using GaussChebychev quadrature and $\delta \theta_{\text {orb }}$ is deduced from Eq. (30).

2. Initialization. The initial angular coordinates $(\phi, \theta)$ of $\boldsymbol{w}=$ $\left(w^{x}, w^{y}, w^{z}\right)$ are computed. We set a variable $L_{2}$ to the total quadratic deflection angle to be covered during $\delta t_{\text {step }}, L_{2} \leftarrow$ $\delta \theta_{\text {step }}^{2}$

3. LC test. If $v^{\mathrm{tg}}=\sqrt{\left(v_{\mathrm{CM}}^{x}+w^{x}\right)^{2}+\left(v_{\mathrm{CM}}^{y}+w^{y}\right)^{2}} \leq v_{\mathrm{LC}} \equiv$ $J_{\mathrm{LC}} / R$, the super-star has entered the loss cone and is disrupted. Otherwise, we proceed to the next step of the procedure. We recall that $\boldsymbol{v}_{\mathrm{CM}}$ is the velocity vector of the pair's center of mass in the cluster reference frame. It is considered constant during the RW process.

4. Completion test. If $L_{2} \leq 0$, the random walk is over. We break from the RW loop, the super-star left unaffected.

5. RW step. A new (meta-)step is realized. First its amplitude is set according to

$\Delta=\max \left(\delta \theta_{\text {orb }}, \min \left(\Delta_{\max }, \Delta_{\text {safe }}, \sqrt{L_{2}}\right)\right)$,

where $\Delta_{\max } \simeq 0.1 \pi$ and $\Delta_{\text {safe }}=c_{\text {safe }}\left(v^{\text {tg }}-v_{\mathrm{LC}}\right) / w$ with $c_{\text {safe }} \simeq 0.2-0.5$. This relation ensures that meta-steps get

7 Actually, as $\delta \theta_{\text {step }} \propto \sqrt{\delta t_{\text {step }}} \propto \sqrt{f_{\delta t}}$, the number of super-stars to be tested for entry into the LC per (mean) $\delta t_{\text {step }}$ scales roughly as $\delta \theta_{\text {step }}^{2} \propto f_{\delta t}$, with $n_{\text {orb }} \propto f_{\delta t}$ steps in each random walk. As the number of $\delta t_{\text {step }}$ needed to simulate the cluster's evolution for a given physical duration is $\propto f_{\delta t}^{-1}$, the total number of RW steps scales as $\propto f_{\delta t}$ and the code gets slower for larger time steps!

${ }^{8}$ More precisely, for planar RW, the length of the surrogate "meta"step should be chosen according to a Gaussian distribution with $n \delta^{2}$ variance.

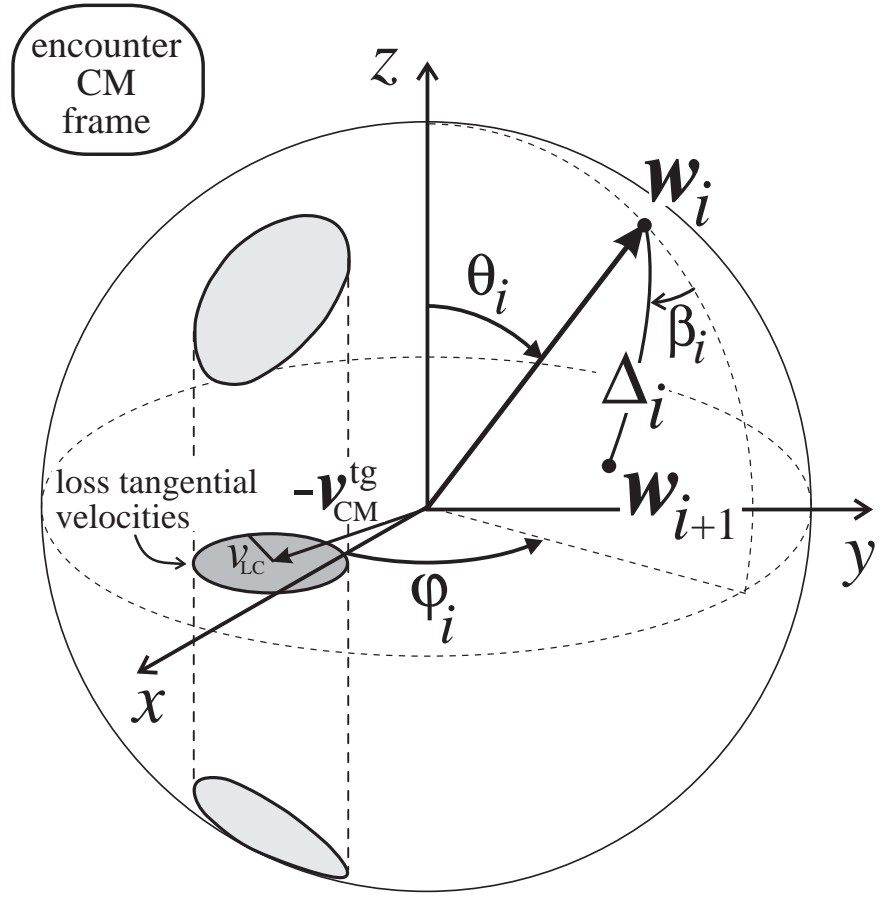

Fig. 4. Geometry of one random walk step on the velocity sphere in the encounter reference frame. $\Delta_{i}$ is the adaptive $i$ th step, $\beta_{i}$ a random angle, $\boldsymbol{w}_{i}$ the particle's velocity after step $i-1$ and $\boldsymbol{w}_{i+1}$ its velocity after step $i$. Velocities with tangential component pointing in the shaded disk correspond to disruption orbits, i.e. with $v^{\mathrm{tg}} \leq v_{\mathrm{LC}} \equiv J_{\mathrm{LC}} / R$ in the cluster reference frame. $\boldsymbol{v}_{\mathrm{CM}}^{\mathrm{tg}}$ is the tangential component of the pair's center of mass velocity.

progressively smaller, down to the "real" individual $\delta \theta_{\text {orb }}$ when the loss cone region is approached during $w$-RW. Then the (meta-)step direction on the sphere is set by an random angle, $\beta$, with uniform $[0,2 \pi$ [ deviate (see Fig. 4). This determines a new orientation $(\phi, \theta)$ for $w$. The remaining quadratic path length is updated, $L_{2} \leftarrow L_{2}-\Delta^{2}$. The loop is closed by branching back to point 3 .

To conclude this section, we highlight some shortcomings in our treatment of the LC. Our procedure amounts to examining whether tidal disruption occurs during the fine-grained diffusion process numerically represented by a single superencounter. Thus, as long as "normal", non-LC relaxation is concerned, the super-encounter and the explicit RW are two statistically equivalent descriptions of the particle's evolution during $\delta t_{\text {step }}$. But only if the RW process leads into the LC, is the particle's $J$ modified as this is needed to determine the outcome of the tidal interaction. Its energy isn't modified accordingly because energy conservation would be violated if some energy change were applied to the super-star without being balanced by an opposite modification for the other super-star that took part to the super-encounter ${ }^{9}$. The main risk is the introduction of some bias in the $E$-distribution of stars that endured

9 Conversely, non-conservation of angular momentum doesn't show up explicitly for the contribution of any super-star to the total $J$ is always zero, by spherical symmetry! However, there is a risk that such "hidden" non-conservations of $J$ may reflect in the distribution of ellipticities by introducing some nonphysical feature in it. 


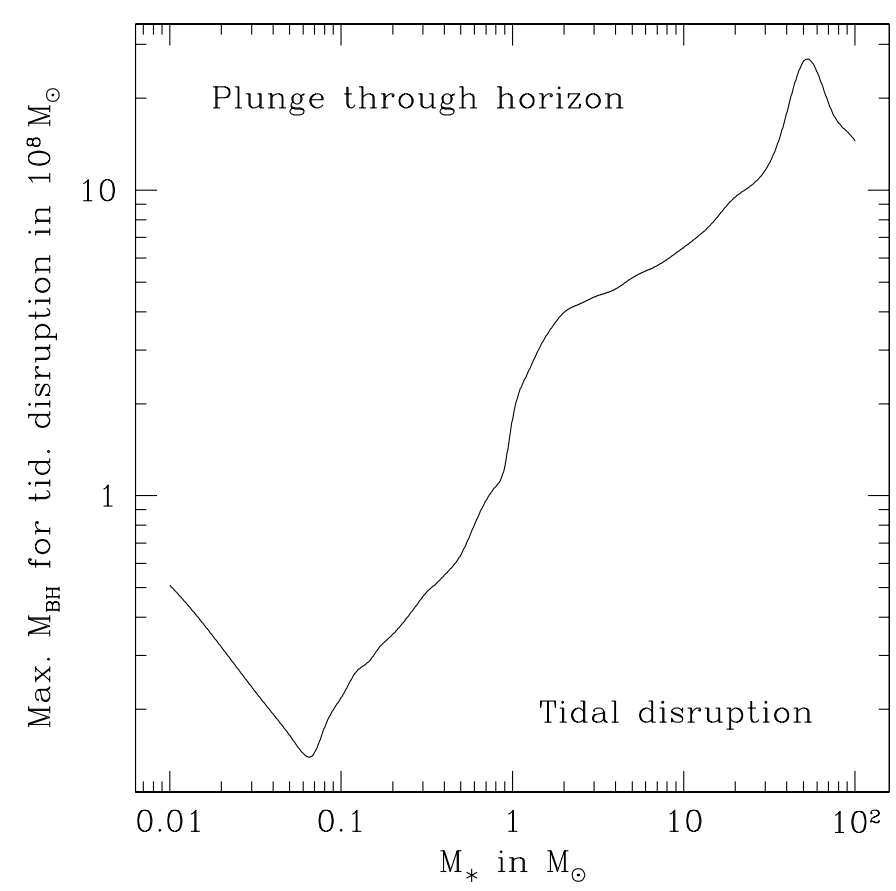

Fig. 5. Maximum mass of the central $\mathrm{BH}$ for tidal disruption, as a function of the mass of of the MS star, $M_{\mathrm{BH}}^{(\max )}=1.6 \times$ $10^{8} M_{\odot}\left(\frac{M_{*}}{M_{\odot}}\right)^{-1 / 2}\left(\frac{R_{*}}{R_{\odot}}\right)^{3 / 2}\left(\frac{R_{\text {plunge }}}{R_{\mathrm{S}}}\right)^{-3 / 2}\left(\frac{\beta_{\mathrm{h}}}{0.8}\right)^{-3 / 2}$. We assumed $R_{\text {plunge }}=R_{\mathrm{S}}$ and $\beta_{\mathrm{h}}=0.8$ and used $M_{*}-R_{*}$ relations from realistic models of MS stars (Schaller et al. 1992; Meynet et al. 1994; Charbonnel et al. 1999; Chabrier \& Baraffe 2000).

partial tidal disruption. Furthermore, if the super-star survived the RW, we give it back the post-super-encounter orbital quantities. Hence, there a possibility that it will be left lying in the LC with no regards to its empty/loss nature! This means that the DF as represented by the code is probably not accurate in the $\mathrm{LC}$ region.

A possible cure to these problems would be to eliminate the super-encounter phase and to perform a symmetric RW for both super-stars at the same time. Unfortunately, this is not so easy for they do not share a common $n_{\text {orb }}$. Also, consistency would dictate to start the random walk with the orbital properties of the super-star (which determine $n_{\text {orb }}$, for instance) before the super-encounter. However, to save computing time, the RW's initial conditions are set to the orbital state modified by the super-encounter, as this spares an extra computation of the peri- and apocenter distances which are needed both to compute $P_{\text {orb }}$ and to select a radial position on the new orbit. Quite unexpectedly, tests have demonstrated that this trick doesn't introduce any significant change in the cluster's evolution (most notably, the BH's growth rate $)^{10}$.

In our description, we neglected the fact that if the $\mathrm{BH}$ is massive enough, its Schwarzschild radius $R_{\mathrm{S}}=2 G M_{\mathrm{BH}} / c^{2}$ can exceed $R_{\text {disr }}$ for stars with a given structure so that they will be swallowed by crossing the horizon without being disrupted. For a star with solar mass and radius, this will happen for $M_{\mathrm{BH}}>1.6 \times 10^{8} M_{\odot}$ while, for giants with $M=1 M_{\odot}$ and

10 To be fair, the gain in speed is also quite modest, as most of computing time is spent in the orbital position selection procedure.
$R=100 R_{\odot}$, only an unrealistic $\mathrm{BH}$ with $M_{\mathrm{BH}}>1.6 \times 10^{11} M_{\odot}$ would be large enough to prevent disruptions from happening. In Fig. 5, we plot, as a function of the mass of the MS star, the maximum $\mathrm{BH}$ mass for which tidal disruption can occur. Note, however, that assuming $R_{\text {plunge }}=R_{\mathrm{S}}$ may be an underestimate. Indeed, a particle with negligible energy at infinity would be pulled into the $\mathrm{BH}$ on a no-return in-spiral orbit by relativistic effects if its specific angular momentum is lower than $J_{\min }=4 G M_{\mathrm{BH}} c^{-1}$, as the effective potential does not have high enough a centrifugal rise. This critical $J$ value corresponds to a parabolic orbit with pericenter separation $R_{\text {plunge }} \stackrel{\text { def }}{=} d_{\text {min }}=4 R_{\mathrm{S}}$ in Newtonian mechanics (Shapiro $\&$ Teukolsky 1983, Sect. 12.3). This should be used as the effective radius of the direct plunge sphere provided tidal disruptions occurring on such relativistic in-spiral orbits do not lead to observable accretion events, i.e. most of the stellar gas stays on in-spiral trajectories, which seems unlikely given the huge spread in orbital energy of the post-disruption gas elements (Rees 1988). An easy modification of the code allows to account for direct plunges but, for the sake of comparison with results from the literature, they were not treated in any simulation presented here.

In the present version of the code, we assume that each time a star enters the disruption sphere, it is completely shredded to gas and that all this gas is immediately accreted onto the central BH. Treating the accretion process as being instantaneous is certainly a good approximation when the mean time between successive disruptions (of order $10^{4}$ years in presentday galaxies) is much longer than the time scale of individual accretion events (a few months to a few years). When this is not the case, one may assume that the gas piles up in some circum$\mathrm{BH}$ reservoir, waiting to be accreted at a later time when the disruption rate has decreased and/or the increased BH mass allows a shorter accretion time (see models à la Murphy et al. 1991 in Sect. 5.3).

On the other hand, assuming complete accretion probably leads to an overestimate of the tidal feeding rate because, due to the huge spread in the energy of debris, only $50 \%$ of the stellar gas is left bound to the $\mathrm{BH}$ just after a complete tidal disruption (Rees 1988; Fulbright 1996, among others). Furthermore, when the leading extremity of this bound gas stream comes back to pericenter, it collides with slower moving material and shocks to such a high thermal energy that of order half of the bound gas may eventually get unbound (Ayal et al. 2000). Consequently, in future works, we should assume that only a fraction $\epsilon_{\mathrm{accr}}=25-50 \%$ of the tidally produced gas is accreted, but, to be consistent with other cluster simulations from the literature, all results reported here were obtained with $\epsilon_{\text {accr }}=100 \%$.

Finally, the assumption of complete disruption is also an over-simplification, as hinted to by, e.g., Fulbright (1996) who showed that the transition regime between no damage and full disruption spans $\beta \simeq 1 \rightarrow 3$ for $n=3$ polytropes. Real MS stars with masses $\geq 1 M_{\odot}$, not to mention giants, are even more concentrated than $n=3$ polytropes so that there is an important range of pericenter distances for which envelope striping, rather than complete disruption would result. Other 
non-disruptive tidal effects like spin-up (Alexander \& Kumar 2001) are also of observational interest for the center of our Galaxy and we plan to extend the abilities of our code in order to be able to keep track of such "tidally perturbed" stars that can amount to an appreciable fraction of the inner stellar population (Alexander \& Livio 2001).

\section{Other additions and improvements}

\subsection{Stellar evolution}

Stellar evolution (SE) is, in principle, an important ingredient to incorporate in nuclei simulations. For a typical IMF, of order $40 \%$ of the Zero-Age MS (ZAMS) mass is lost from the stars in the first $10^{10}$ years, so SE is potentially one of the dominating source of fuel for the BH. Also, how stars are affected by relaxation, collisions and tidal disruptions obviously depends on their masses and radii. For example, compact remnants resist disruptive events and, with the help of mass segregation, may come to dominate the central regions. Whether or not larger and larger stars may be formed through successive mergers also depends crucially on the relative time scales of stellar evolution and collisions.

For the time being, our treatment of SE is simple-minded and straightforward. We assume that a star is "born" on the ZAMS and keeps the same mass and radius during its MS life which is of duration $T_{\mathrm{MS}}$. We use the relation $T_{\mathrm{MS}}\left(M_{*}\right)$ given by Bressan et al. (1993). When it leaves the MS, this star is immediately turned into a compact remnant, according to the following prescription (Miralda-Escudé \& Gould 2000). All progenitors with masses lower than $8 M_{\odot}$ become $0.6 M_{\odot}$ white dwarfs, those with masses $8-30 M_{\odot}$ become $1.4 M_{\odot}$ neutron stars and those with larger masses become $7 M_{\odot}$ BHs. Part of the emitted gas is accreted on the central $\mathrm{MBH}$ and the remaining is ejected from the cluster. This simplistic relation between the ZAMS mass of a star and the final product of its evolution mainly reflects the lack of a strong set of observational constraints or theoretical predictions in this domain. In any case, it is known that the ZAMS $\longrightarrow$ remnant relation strongly depends on metallicity, if only because stellar winds do (Maeder 1992). All in all, it appears to us that these aspects of SE are probably a main source of uncertainties affecting the prediction of stellar dynamical mechanisms in which remnants take an important part.

SE introduces a new time scale, namely $T_{\mathrm{MS}}$ in the present implementation. To resolve it correctly, we impose the time step $\delta t(R)$ to be smaller than a fraction $f_{\delta t}^{(\mathrm{SE})}$ (typically 0.05 ) of the minimum of $T_{\mathrm{MS}}$ as evaluated in each cell of the same radial mesh we use to estimate $T_{\text {rel }}(R)$ and $T_{\text {coll }}(R)$. But, contrary to relaxation and collisions, in the absence of a strong initial mass segregation, there is no reason for this time-scale to increase with increasing $R$. Consequently, when SE proceeds faster than other processes, it imposes (nearly) the same, very short $\delta t$ to all super-stars and we loose the advantage of $R$-dependent $\delta t$. In the simulations we have performed so far with SE included, we assumed a unique initial episode of star formation a $t=0$ so that, as soon as high mass stars have been turned into remnants, the slowing down due to stellar evolution ceases and the total CPU time is only increased by a factor of a few. A more fearsome performance decline will result if some form of continuous stellar formation is simulated or if the red giant phase has to be resolved as well.

\subsection{Particle doubling}

To maintain a high resolution in the late evolutionary stages of a highly collisional, disruptive or evaporative cluster, we resort to particle doubling. When the number of remaining superstars has reached half the initial number, every super-star is split into two copies with the same orbital and stellar properties. In the first stage of the procedure, both copies are left at the same position $R$ where their "parent" was. Then, we pick each super-star in turn, in random order, and place it at a random position on its orbit, in a way identical to what is done at the end of a normal evolutionary step. In that way, we minimize the risk of maintaining potentially harmful correlations between super-stars descending from a common ancestor. Of course, after particle doubling, the number of stars represented by each super-star has to be divided by 2 . Some cluster models (like the one set according to DS82 model E, see below) go through several episodes of particle doubling. Implementing proper book-keeping was the main difficulty with this new, otherwise straightforward, feature.

\subsection{Miscellaneous}

Various minor improvements have also been recently added to the code. For instance, in order to ensure that the orbital parameters $(E$ and $J)$ and positions of the super-stars are given time to adapt to the (supposedly adiabatic, see Sect. 5.1) modification of the potential, we force time steps $\delta t(R)$ to be smaller than some fraction $f_{\text {evap }}$ of the evaporation time, $T_{\text {evap }} \stackrel{\text { def }}{=}$ $M_{\mathrm{cl}}\left(\mathrm{d} M_{\mathrm{cl}} / \mathrm{d} t\right)^{-1}$ where $M_{\mathrm{cl}}$ is the stellar mass of the cluster, and smaller than some fraction $f_{\text {int }}$ of the "intern mass evolution" time $T_{\text {int }}(R) \stackrel{\text { def }}{=} M_{\text {int }}(R)\left(\mathrm{d} M_{\mathrm{cl}} / \mathrm{d} t\right)^{-1}$ where $M_{\text {int }}(R)$ is the total mass interior of $R$. Typically, values around 0.01 are used for $f_{\text {evap }}$ and $f_{\text {int }}$.

Also, in addition to the usual test we perform each time a particle has to be evolved, we periodically check for all the super-stars to be bound. This is an iterative procedure because if, during the first pass, we detect super-stars that are unbound, we remove them from the system and this may unbound other particles.

\section{Test simulations}

\subsection{Adiabatic adaptation of the star cluster to the growth of a central black hole}

In some instances the central $\mathrm{BH}$ can grow significantly on a time scale $T_{\mathrm{BH}}$ much longer than the cluster's dynamical time but still much shorter than relaxation time. Such a hierarchy naturally occurs if a substantial amount of gas is flowing into the $\mathrm{BH}$ from outside the nucleus. Quick $\mathrm{BH}$ growth can also happen if mass lost my stars, either due to normal stellar 
(a)

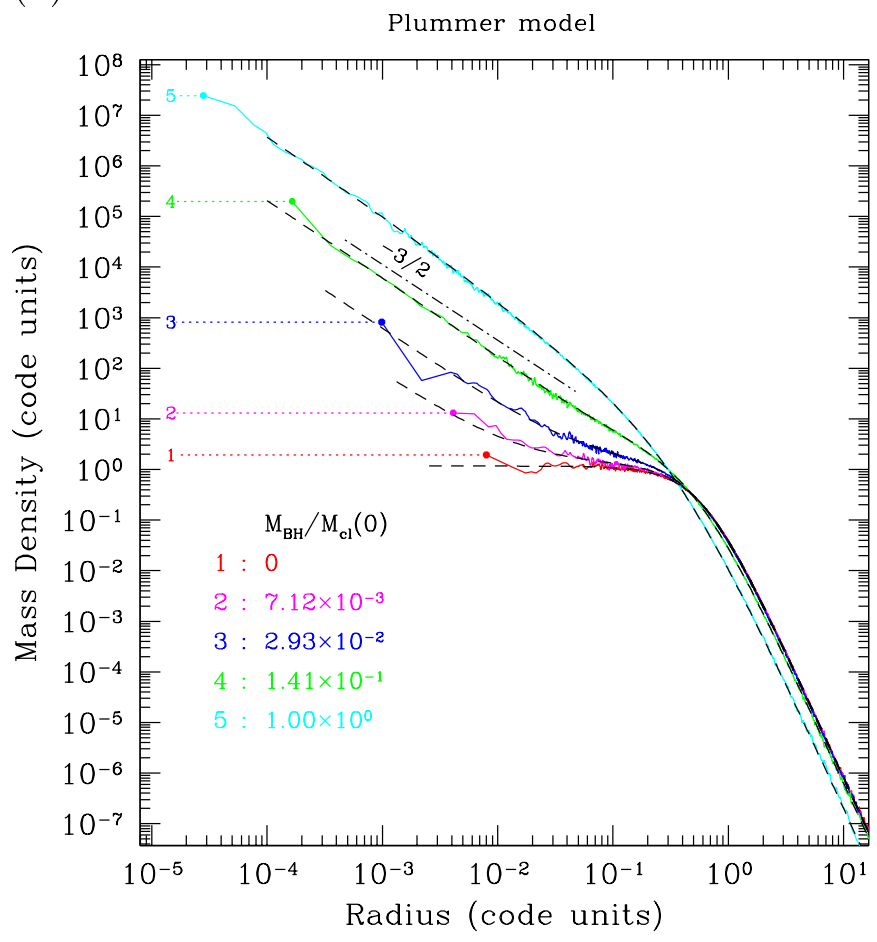

(c)

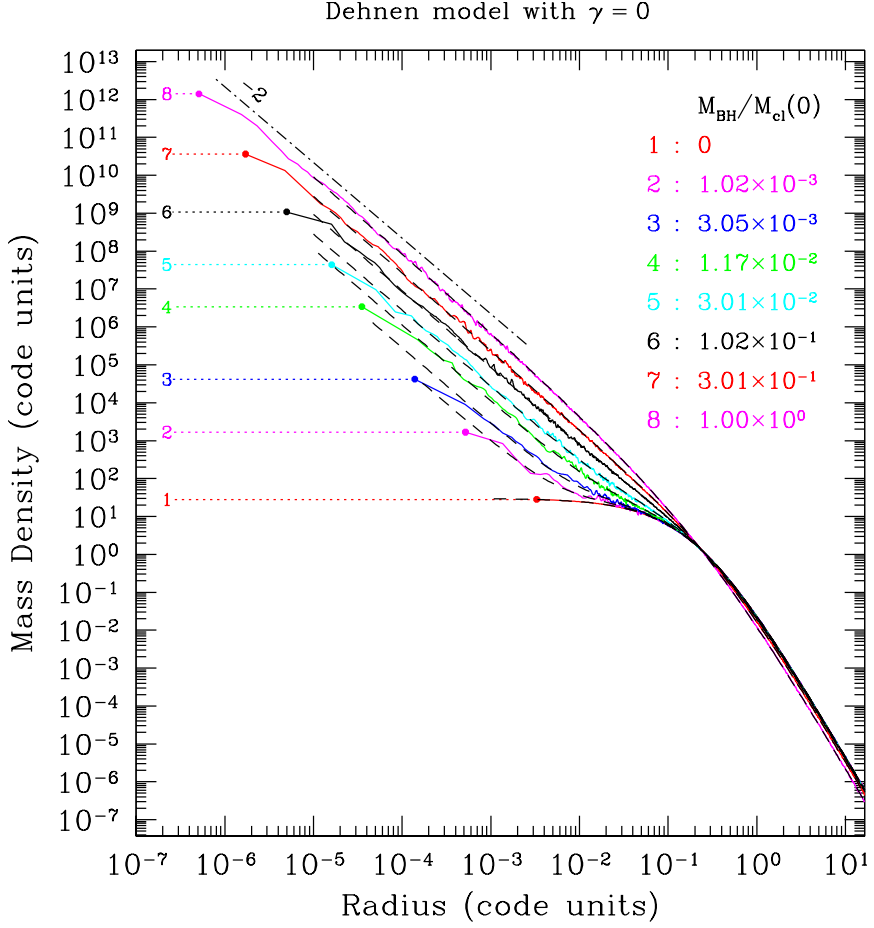

(b)

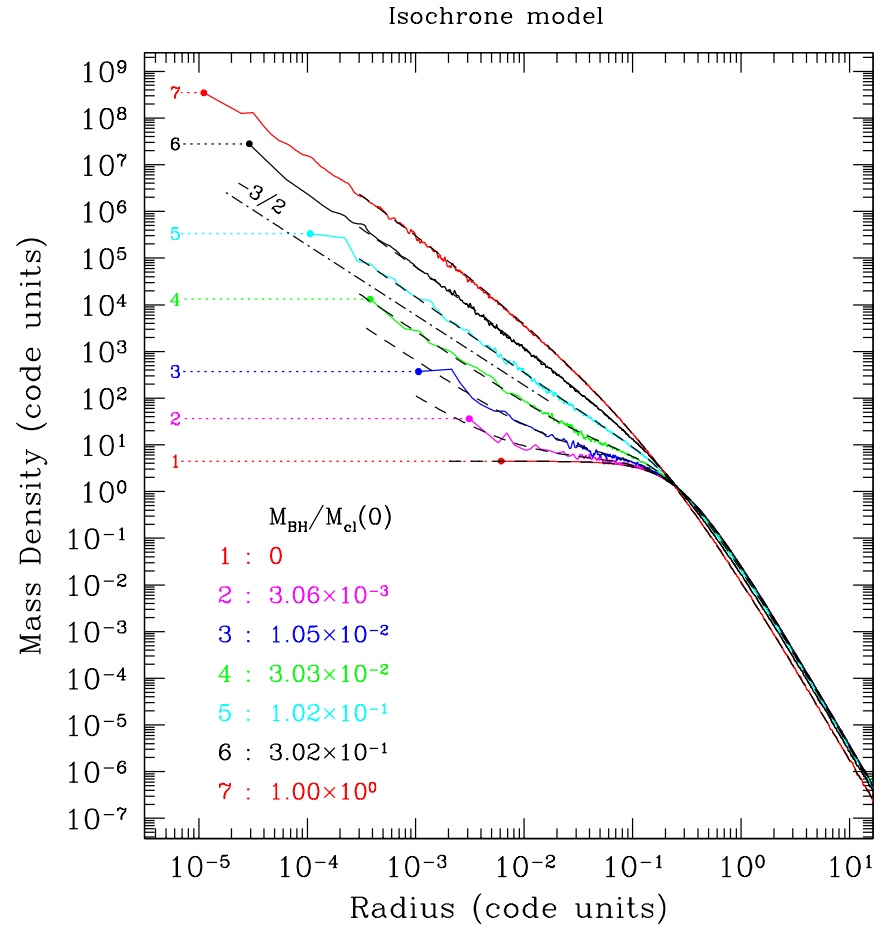

(d)

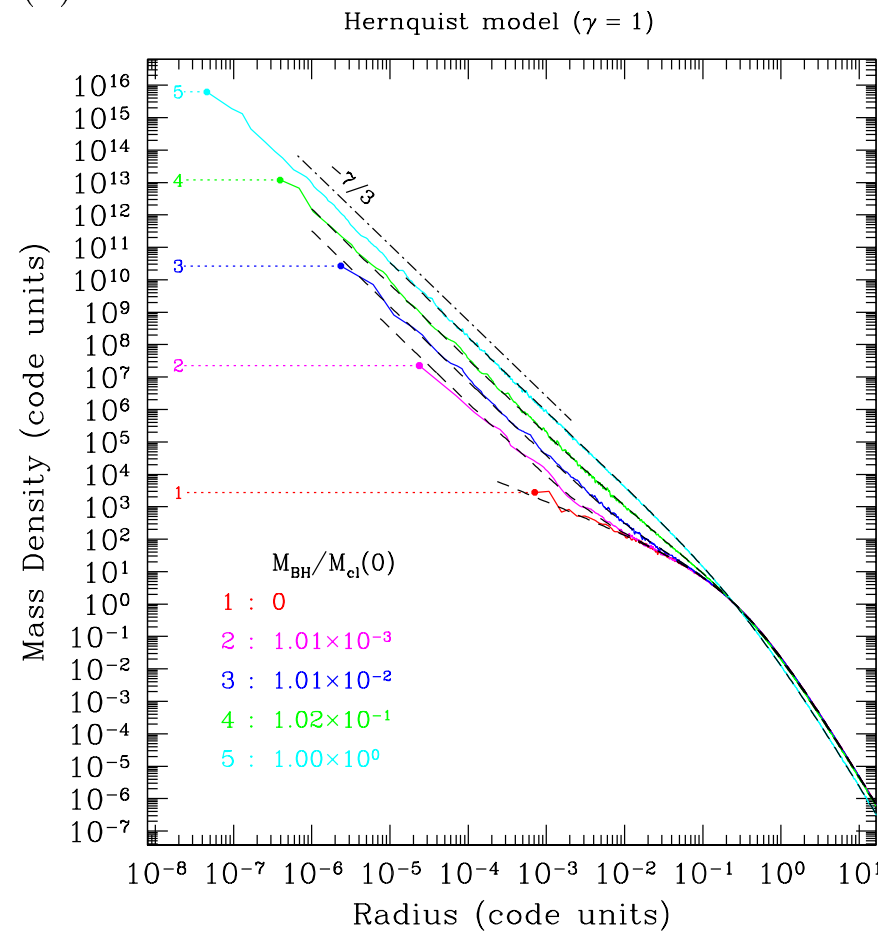

Fig. 6. Adiabatic growth of a central BH in various cluster models. Evolution of the stellar density. Jagged solid lines are results of our MC simulations with $10^{6}$ super-stars. Smooth dashed lines are theoretical predictions based on the conservation of angular momentum and radial action. They have been computed with a code provided by G. Quinlan (Quinlan et al. 1995). The dot-dashed line segment indicates the asymptotic cusp slope from Eq. (32). It applies for $M_{\mathrm{BH}}<M_{\mathrm{cl}}$. a) Plummer model. b) Isochrone model. c) $\gamma$-model with $\gamma=0$. d) Hernquist model. The agreement between the MC results and the theoretical predictions is excellent. 
(a)

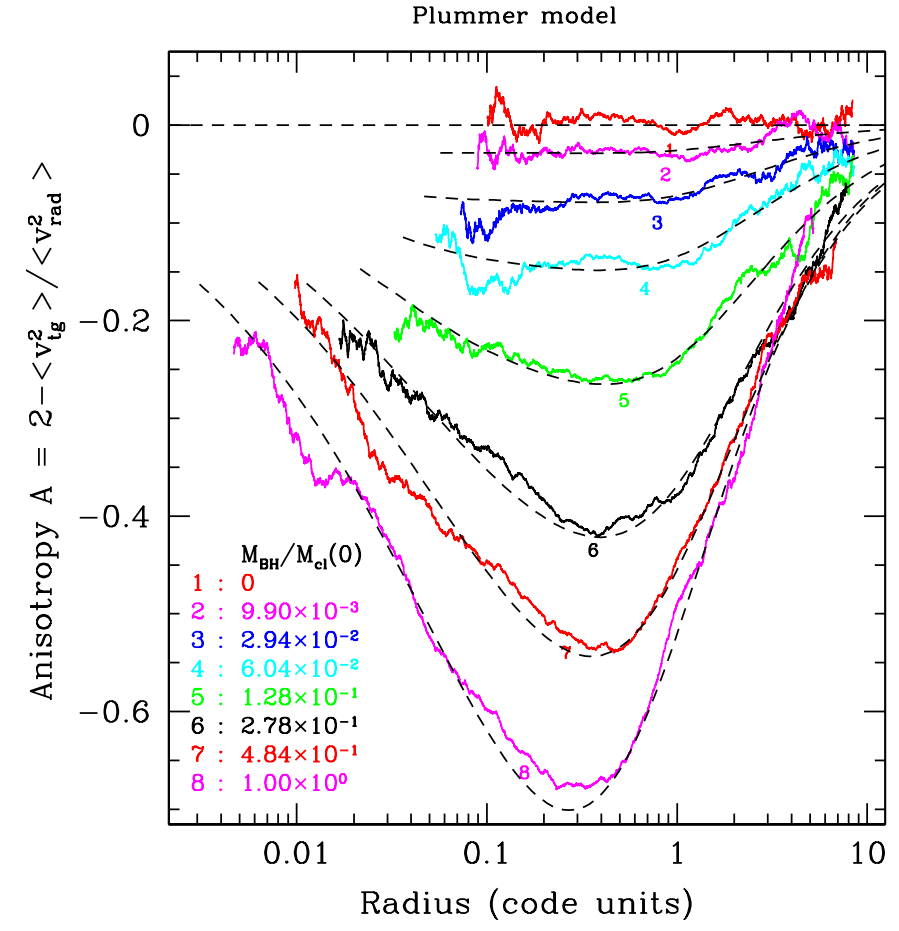

(b)

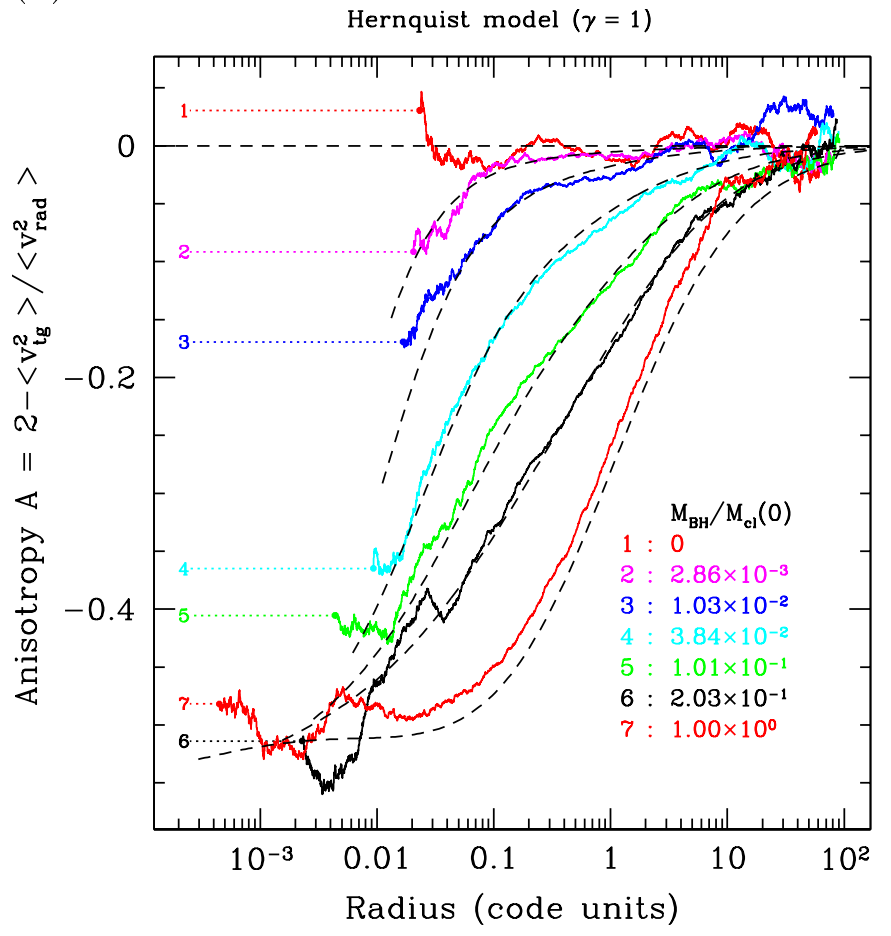

Fig. 7. Adiabatic growth of a central BH in, a) a Plummer model and, b) a Hernquist model. Evolution of the velocity anisotropy. Solid lines are our results, dashed lines are theoretical predictions from the code of Quinlan et al. (1995). For the sake of display clarity, snapshots selected here are different from those in Fig. 6. Our curves have been smoothed with a sliding averaging procedure. To cover a larger range in radius, the average is done over a smaller number of super-stars at small and large radii than at intermediate positions. Given the high level of noise in the MC data, the agreement with Quinlan's predictions is very satisfactory until $M_{\mathrm{BH}}$ grows past $0.8 M_{\mathrm{cl}}$. From this time, the tangential anisotropy in the outer parts of our models fails to increase with larger BH masses (see text).

evolution (in a young cluster), or to disruptive collisions (in a very dense cluster), is efficiently accreted on the BH.

As a consequence of the slow modification of the potential, the shape of stellar orbits evolve while conserving adiabatic invariants, i.e. the angular momentum $J$ and the radial action $I_{\mathrm{R}}$ (Young 1980; Binney \& Tremaine 1987). Correspondingly, the density profile of stars around the BH and their velocity distribution are modified. Characteristics of the resulting stellar profiles have been worked out for various initial clusters, either semi-analytically, using the conservation of the DF when expressed as a function of adiabatic invariants (Young 1980; Lee \& Goodman 1989; Cipollina \& Bertin 1994; Cipollina 1995; Quinlan et al. 1995) or by means of $N$-body simulations (Sigurdsson et al. 1995; Leeuwin \& Athanassoula 2000).

These studies show that a power-law cusp develops inside the influence sphere of the $\mathrm{BH}$, of radius $R_{\bullet}$, in which $G M_{\mathrm{BH}} / R$ exceeds the original velocity dispersion of the stars. According to Quinlan et al. (1995), if the initial stellar cluster is isotropic, presents a density cusp $\rho \propto R^{-\gamma_{\mathrm{i}}}$ with $\gamma_{\mathrm{i}} \geq 0$ and a DF diverging near $E=\phi(0)$ like $f(E) \propto(E-\phi(0))^{-n}$, then the final density cusp has an exponent

$\gamma_{\mathrm{f}}=\frac{3}{2}+n\left(\frac{2-\gamma_{\mathrm{i}}}{4-\gamma_{\mathrm{i}}}\right)$.

This result only applies very close to the $\mathrm{BH}$ if its mass is larger than the mass of the initial stellar core; the cusp may be steeper at intermediate distances, $R_{\text {trans }}<R<R$. with
$R_{\text {trans }}=R_{\mathrm{c}}^{2} / R$. (Lee \& Goodman 1989; Cipollina \& Bertin 1994, see also Leeuwin \& Athanassoula 2000). Another key feature is the development of noticeable tangential anisotropy in the central regions. In models with analytic cores (i.e. with $(\rho(0)-\rho(R)) \propto R^{2}$ near the center), this anisotropy, although it is caused by the central $\mathrm{BH}$, does not actually appear in the center itself where isotropy is conserved (Goodman \& Binney 1984; Quinlan et al. 1995).

We have performed simulations of the adiabatic growth of a central BH in a variety of cases. In addition to the traditional Plummer model, we adopted the same set of models as Quinlan et al. (1995). These are the isochrone cluster (Hénon 1959, 1960; Binney \& Tremaine 1987), which has an analytic core, and three " $\gamma$-models" (Dehnen 1993; Tremaine et al. 1994) whose density profile is

$\rho_{\gamma}(R)=\frac{3-\gamma}{4 \pi} \frac{M_{\mathrm{cl}} R_{\mathrm{b}}}{R^{\gamma}\left(R+R_{\mathrm{b}}\right)^{4-\gamma}}$

where $R_{\mathrm{b}}$ is the break radius. The used $\gamma$ values are 0,1 (Hernquist 1990) and 2 (Jaffe 1983). None of these models has an analytic core. Equation (32) predicts $\gamma_{\mathrm{f}}=3 / 2,3 / 2,2,7 / 3$ and 5/2 for Plummer, isochrone, and $\gamma=0,1,2$ models, respectively (Quinlan et al. 1995).

To simulate the process of adiabatic $\mathrm{BH}$ growth, we switched off relaxation and all the other physical processes in the MC code. The algorithm reduces then to moving super-stars on their orbits again and again (see Sect. 5.2 of Paper I) while 
$M_{\mathrm{BH}}$ is slowly increased. The time step condition is $f_{\text {int }}=0.002$ (see Sect. 4.3). This relatively small value is required to get a correct evolution of the anisotropy in the outer parts of the cluster. With larger time steps, the particles at large radii react too impulsively to the BH's growth and their orbits tend not to develop enough tangential anisotropy or even to become radially dominated. Note, however, that this problem only occurs when the BH's mass is larger than half the mass of the stellar cluster and that the density profile appears to be unaffected by this even for $f_{\text {int }}=0.01$.

In Fig. 6, we compare our results with the output of the code written by Quinlan et al. (1995) and kindly provided by van der Marel. This code makes explicit use of the conservation of adiabatic invariants to determine the structure of the $\mathrm{BH}$-embedding cluster and we can regard its results as secure predictions. As can be seen on these diagrams, the MC code behaves very nicely in this regime. Given the numerical noise to be expected from such a method, the density profiles are deemed to be in perfect agreement for all models. In Fig. 7, the evolution of the anisotropy profile for the Plummer and the Hernquist model is plotted. This quantity, when determined from MC results, suffers from a much higher statistical noise, so that a stronger smoothing must be applied to get useful curves. Despite this noise, it is quite clear that our results match the predictions very well, except for the outer parts that lack some tangential anisotropy for large $M_{\mathrm{BH}}$, as already discussed.

\subsection{Cluster models with tidal disruptions}

Another idealized regime to which many theoretical and numerical studies have been devoted is the case of a the relaxed single-mass spherical stellar cluster with a central $\mathrm{BH}$. Collisions are neglected but stars entering the tidal disruption region are destroyed and their mass is added to the $\mathrm{BH}$. Bahcall \& Wolf (1976) demonstrated that the quasi-steady state solution of the Fokker-Planck equation for this situation corresponds to a central density cusp with $\rho \propto R^{-7 / 4}$. Although these authors used an one-dimensional approach with the energy $E$ as the only variable, more accurate numerical integrations of the stationary FP equation in $(E, J)$ space, with a proper account of loss cone effects, have confirmed this result (Lightman \& Shapiro 1977; Cohn \& Kulsrud 1978), as did evolutionary models (Duncan \& Shapiro 1983, hereafter DS83, for instance). As testified by Fig. 10, we reproduce this result. This plot shows the evolution of a model with same physics and initial parameters as model I of DS83 and is described in more details in the next sub-section.

A few evolutionary models have been published that are based on these simple physical assumptions. Most were meant to explore the possibility of forming a $\mathrm{MBH}$ during the core collapse of a globular cluster ${ }^{11}$. They usually start with a seed

11 However, neglecting the role of a mass spectrum and binary stars, they fall short of physical realism. Unless the cluster is born with a very high velocity dispersion, $\sigma_{\mathrm{v}}^{2} \propto M / R \gg V_{\text {orb }}^{2}$, where $M$ is the total mass, $R$ a measure of the size of the cluster and $V_{\text {orb }}$ a typical value for the (internal) orbital velocity of binaries (an unrealistic assumption

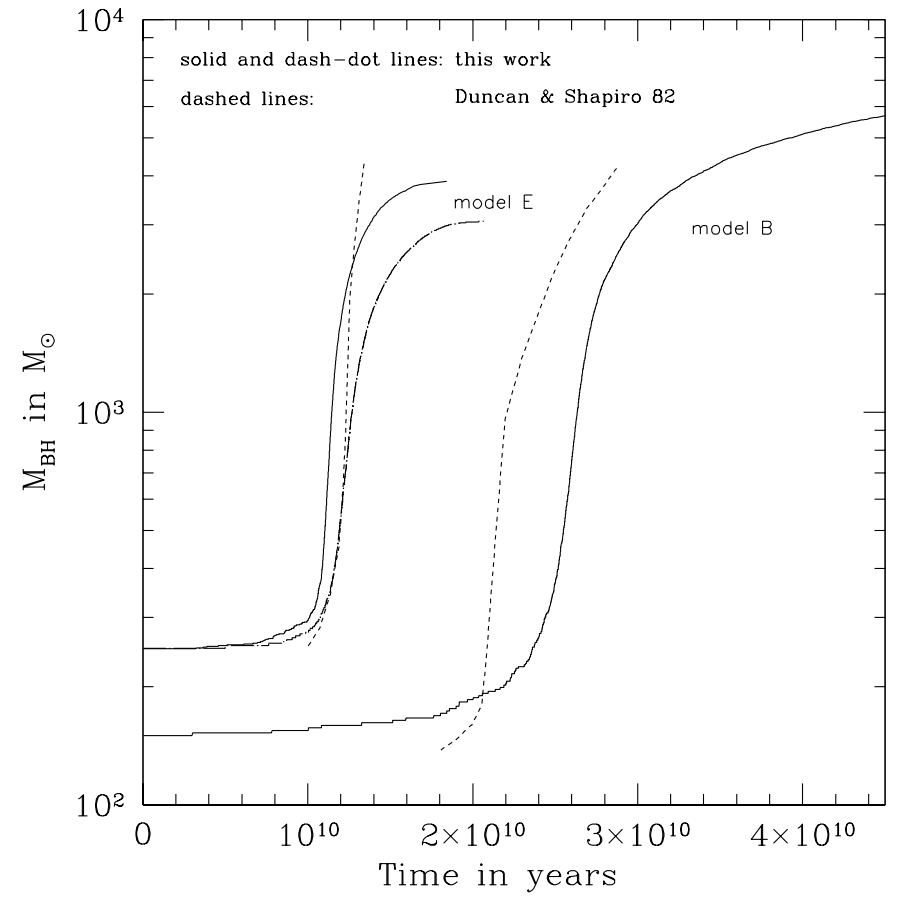

Fig. 8. Growth of the central $\mathrm{BH}$ for models with initial conditions similar to models B and E of Duncan \& Shapiro (1982). Our results, obtained with $256 \mathrm{k}$ super-stars, (solid and dash-dotted lines) are compared with those of these authors (dashed lines). We made two simulations of model E. Both have been stopped when the stellar cluster was reduced to $500 M_{\odot}$. In the first one (solid line), we start abruptly with a tidal radius smaller than the cluster which rapidly adapt to this truncation. In the second run (dash-dotted line), we let the cluster adapt gently to the tidal truncation before we actually start the simulation by switching on relaxation (see text).

black hole which grows by consuming stars. To check the tidal disruption rate given by our code, we compare the growth of the central $\mathrm{BH}$ in such models with results from the literature.

Figure 8 shows such a comparison for models B and E of Duncan \& Shapiro (1982, hereafter DS82) to whom we refer for the specification of initial and boundary conditions. We have used the same setting as these authors except that, in our computations, there is no initial stellar cusp around the $\mathrm{BH}$ and that, for model $\mathrm{B}$, the $\mathrm{BH}$ is present from the beginning of the simulation and not added at a later time as done in DS82. We don't think these minor changes have any significant effect because the initial $\mathrm{BH}$ amounts to only a tiny fraction of the cluster's mass $\left(M_{\mathrm{BH}}=150,250 M_{\odot}\right.$, respectively, with $\left.M_{\mathrm{cl}}=3 \times 10^{5} M_{\odot}\right)$. The match between our results and those of DS82 is not very good. In particular, for model B, the BH's growth starts at a significantly later time but produce an object of comparable mass. However, DS82's simulations were stopped shortly after core rebound, which does not allow a comparison at late times. Note that the growth starts when core

for globular cluster but which may apply to models of proto-nuclei of galaxy like those of Quinlan \& Shapiro 1990), the binaries will delay collapse and probably trigger core rebound before the central density is high enough for efficient "tidal feeding" of a seed BH (see Gao et al. 1991; Giersz \& Spurzem 2000; Giersz 2001; Rasio et al. 2001, for simulations of globular clusters with primordial binaries). 
collapse is sufficiently deep to bring many stars close enough to the $\mathrm{BH}$ to be disrupted and that it is stopped by the fact that the disruption of these stars, most of which have large negative energies, amounts to heating the stellar cluster. Consequently, the temporal shift between DS82's growth curve and ours mostly reflects that our code predicts a longer core-collapse time, $T_{\mathrm{cc}}$. We refer to Paper I for a discussion of this point and the large spread found in the literature for the value of $T_{\mathrm{cc}}$.

Concerning model $\mathrm{E}$, on the one hand, our value for the time of strongest growth, again a quantity nearly coincident with $T_{\text {cc }}$, nicely agrees with DS82. Note that this cluster, being a Plummer with a strong tidal truncation, evolves quicker and differently than an isolated cluster, which gives more weight to this agreement. At the end of our simulations, around 20 Gyrs, the cluster has nearly completely evaporated. On the other hand, the BH's growth is steeper and stronger in DS82's simulation. There is no doubt that it would have produced a significantly larger final $\mathrm{BH}$ than in our case, had their simulation been carried on up to cluster dissolution. The reason for this disagreement is not known to us. We suspected that it may be linked to the fact that, in our simulation, the remaining cluster mass is lower at all times than in DS82, which may, in turn, be due to the way our and DS82's code cope with the strongly out-of-equilibrium initial conditions. Indeed $\sim 10 \%$ of all stars are initially beyond tidal radius. In our model, the cluster loses $17 \%$ of its super-stars very quickly to adjust to the tidal truncation. To have a better handle on this problem, we re-made the simulation with a cluster model which was first allowed to settle to equilibrium with its tidal truncation. To do this, we "evolved" it with no relaxation or any other physical process but still moving super-stars on their orbits in the usual way. If a selected super-star was found with apocenter beyond tidal radius, it had only a small probability (around 0.01) to be removed at this step and was otherwise kept (at the same position). We think that this method produces a better initial structure in which each super-star has been given time to react "adiabatically" to the enforcement of the tidal truncation. $15 \%$ of the cluster mass is lost in this procedure and the resulting cluster also shows less evaporation during its further, relaxation-driven, evolution. However, this does only increase the discrepancy with DS82 concerning the final mass of the $\mathrm{BH}$, see the dash-dotted curve in Fig. 8. Our higher evaporation rate is probably due to our simpler prescription for escape. We immediately remove any super-star which gets on an orbit with apocenter distance beyond tidal radius, regardless of its actual position on this orbit. More realistically, DS82 allowed stars on escape orbits to be kicked back to bound orbits. Recent works (Fukushige \& Heggie 2000; Takahashi \& Portegies Zwart 2000; Baumgardt 2001) made it clear that evaporation from a cluster with a relatively low number of stars can not be regarded as instantaneous: it takes of order one orbital time for a star to actually leave the cluster and the probability for it to be back-scattered onto a bound orbit is non vanishing. Whether or not some improvement in the line of this in our evaporation prescription would lead to a better agreement with DS82 concerning $M_{\mathrm{BH}}(t)$ is not obvious as these two aspects may well be uncoupled. Note that a similar mismatch in the BH's growth curve appears in comparisons with prelimi-

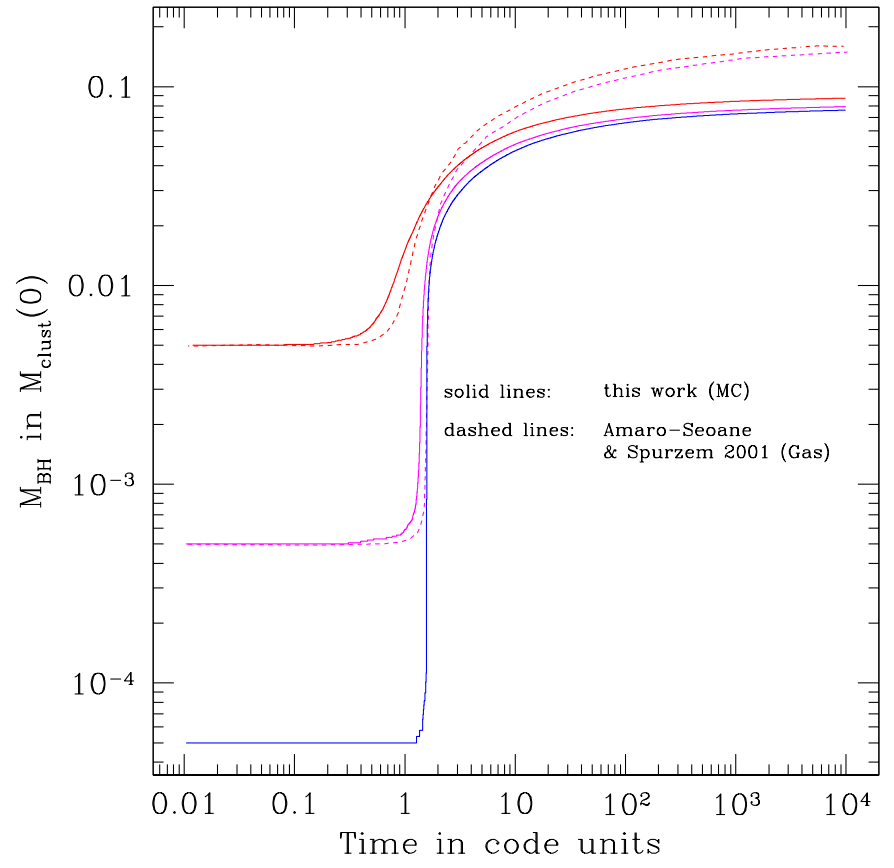

Fig. 9. Growth of the central $\mathrm{BH}$ for models with initial conditions identical to those of Amaro-Seoane \& Spurzem (2001). Our results, obtained with 256k super-stars, (solid lines) are compared with those of these authors (dashed lines).

nary simulations realized by Amaro-Seoane \& Spurzem (2001) with a gas code (see below), but doesn't show up in comparisons with other results obtained by Duncan \& Shapiro (1983) with their MC code and by Murphy et al. (1991) with a direct Fokker-Planck scheme (see next subsection).

In Fig. 9, we display the growth of the central BH for clusters corresponding to the models used by Amaro-Seoane \& Spurzem (2001, hereafter AS01). These consist of $10^{5} 1 M_{\odot}$ stars distributed according to a Plummer density law with a core radius of $0.707 \mathrm{pc}$. The cluster is seeded by a fixed central $\mathrm{BH}$ with an initial mass of 5,50 or $500 M_{\odot}$. Only the last 2 values have been used by AS01. It is clear that for masses as low as 5 or even $50 M_{\odot}$, neglecting the motion of the $\mathrm{BH}$ is quite an unphysical assumption which is required by the present limitations of numerical codes. Even if a close agreement is not reached, our results are very similar to the curves from AS01. In particular, we get the same phenomenon of convergence at late times toward an unique value of $M_{\mathrm{BH}}$. This value is however smaller by a factor of $\sim 2$ than that of AS01.

\subsection{Galactic nucleus models including collisions}

After having checked individual aspects of the MC code in simplified models (pure relaxation in Paper I, collisions rates in Sects. 2.2 and 2.3, adiabatic BH growth in Sect. 5.1...), we turned to the few published works addressing the long term evolution of dense galactic nuclei in order to check our code's global behavior in physical regimes more relevant to our astrophysical field of interest.

We first wanted to avoid the extra complication of stellar evolution and discarded those papers which take it into account. 


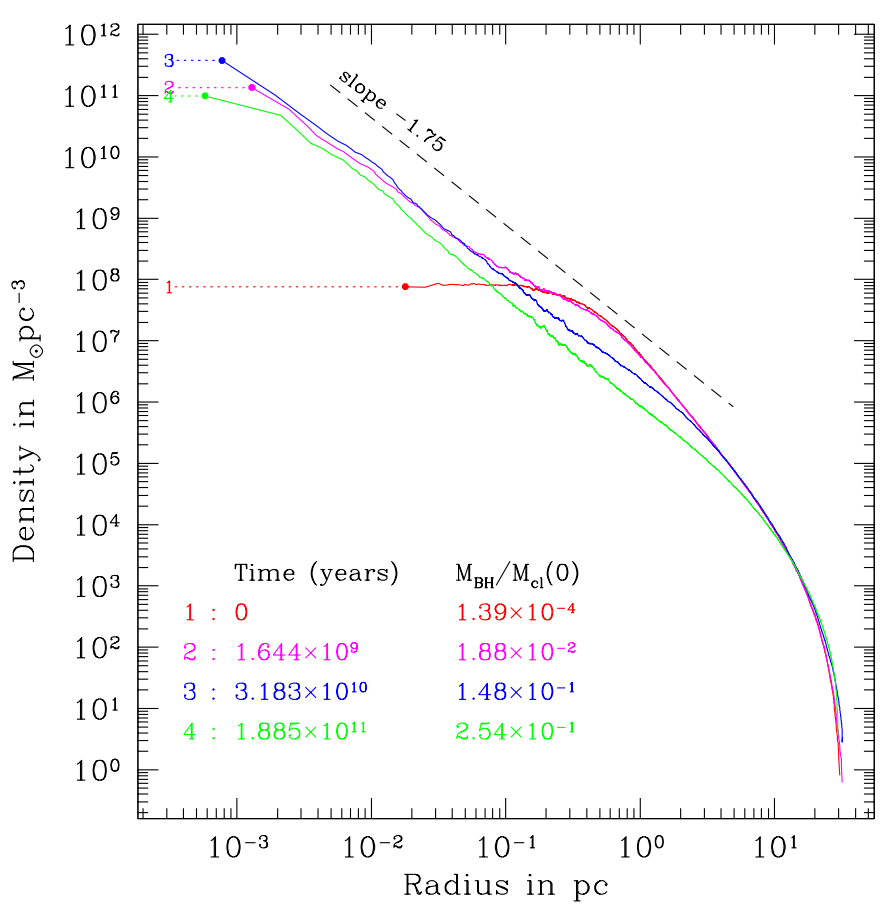

Fig. 10. Evolution of the density profile for a cluster with initial conditions identical to models I/II of DS83. The initial number of superstars is $10^{6}$. As in DS83's model I, collisions are not simulated. One notes the rapid development of a $\rho \propto R^{-1.75}$ cusp.

Furthermore, by their nature, Fokker-Planck methods can only include collisional effects in an approximate way so that they don't allow a clear check of this aspect of the code. Finally, $N$-body simulations (Arabadjis 1997; Rauch 1999), although much more realistic ${ }^{12}$, were deemed too noisy to provide reliable data to compare with.

So we chose the venerable models by Duncan \& Shapiro (1983, hereafter DS83) to conduct tests that include relaxation, tidal disruptions and stellar collisions. DS83 studied three different models. The initial structure is a King cluster with $W_{0}=8$ made of identical stars with $M_{*}=1 M_{\odot}$. Models I and II share the same initial conditions: $3.6 \times 10^{8}$ stars and a core radius $R_{\mathrm{c}}=0.50 \mathrm{pc}$ (the total radius is $34.7 \mathrm{pc}$ ). A seed black hole is present at the center with an initial mass $M_{\mathrm{BH}}(0)=5 \times 10^{4} M_{\odot}$. Model III was devised to reach quasarlike accretion rates. It initially contains $57 \times 10^{8}$ stars, it has $R_{\mathrm{c}}=0.82 \mathrm{pc}$ and $M_{\mathrm{BH}}(0)=2 \times 10^{6} M_{\odot}$. Models II and III include stellar collisions. They are assumed to be completely disruptive and the gas they release is instantaneously and completely accreted on the $\mathrm{BH}$. We used the same initial conditions and physics but, to assess the influence of the assumption of complete collisional destruction, we carried out two extra simulations using our realistic, SPH-generated, prescriptions (models IIb and IIIb).

In Figs. 10 and 11, we present the evolution of the density profile for models I and II, respectively. The most conspicuous feature of the first figure is a spreading central cusp with

\footnotetext{
12 In the case of Arabadjis (1997), it is not clear, however, how reliably relaxation processes can be simulated with a TREE algorithm.
}

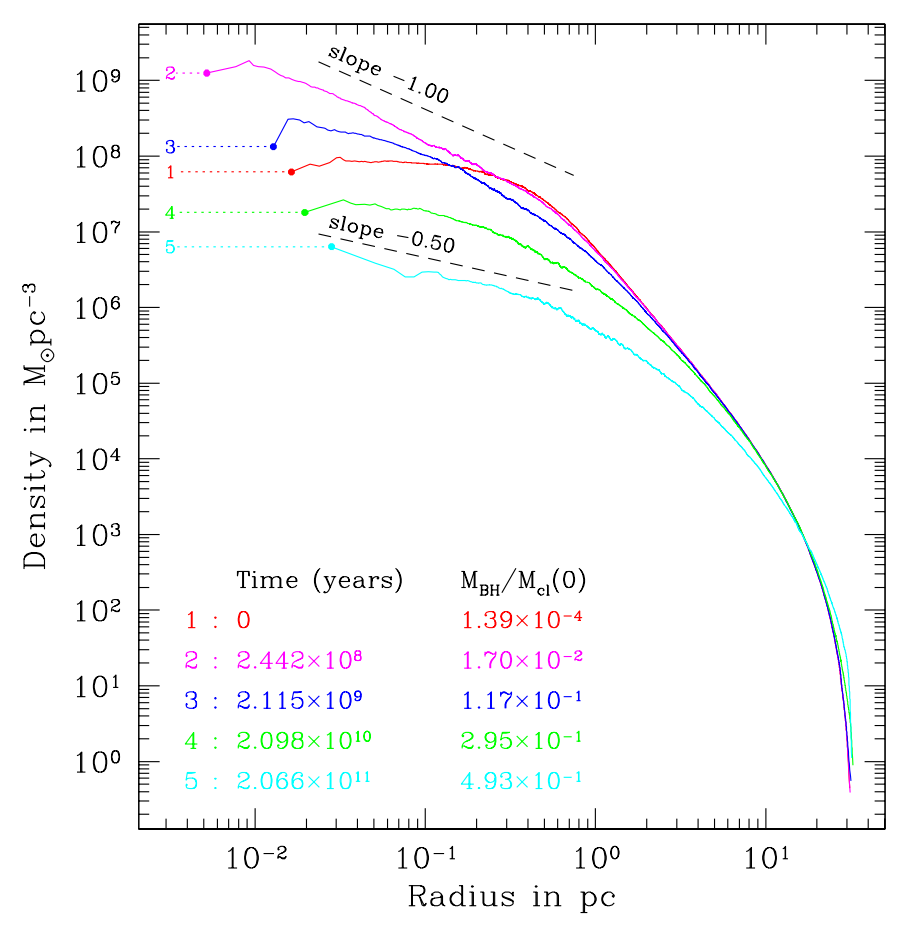

Fig. 11. Evolution of the density profile for a cluster with initial conditions identical to models I/II of DS83. The initial number of superstars is $2 \times 10^{6}$. As in DS83's model II, collisions are simulated. They are assumed to be completely disruptive. Instead of a steep $\rho \propto R^{-1.75}$ power law, the cusp in the center gets milder and milder.

$\rho \propto R^{-7 / 4}$. Such a power-law profile is reproduced here for the first time by a Hénon-like Monte Carlo method. Figure 11 shows that when disruptive collisions are introduced in our calculations, as in DS83's model II, a much milder cusp first appears (with exponent $\sim$ 1) and progressively flattens (with exponent $\geq-0.5$ ). It has been repeatedly reported that collisions strongly decrease the steepness of the inner density profile (Duncan \& Shapiro 1983; Murphy et al. 1991; David et al. 1987a, b; Rauch 1999). A slope of $\sim-0.5$ is often obtained. However, the simulations by Rauch (1999) point to the establishment of a flat, cusp-less central region, not unlike our own results. Murphy et al. (1991) get a strong depletion of stars in the innermost part of the cluster, a result which is apparently reproduced in some of Rauch's models. For lack of resolution, there is no similar effect to be seen in our simulations. The practical relevance of this discrepancy is probably low, however, because the size of this rarefied zone is so small that it would contain only a few $M_{\odot}$ in most cases even without depletion. So the validity of a statistical treatment of such a tiny region is highly questionable anyway. The evolution of the density profile for model III is qualitatively similar. Interestingly, model IIb, which incorporate realistic, partially disruptive collisions also forms a $R^{-7 / 4}$ cusp, but in the much denser model IIIb, collisions are efficient enough to reduce the exponent to a value between -1 and -0.5 .

The growth rate of the $\mathrm{BH}$ is depicted in Fig. 12. The qualitative agreement with DS83 is satisfying even though the rate we obtain is higher by a factor of $\sim 2$ in initial phases of collisional models. The reason of this difference is unknown to 


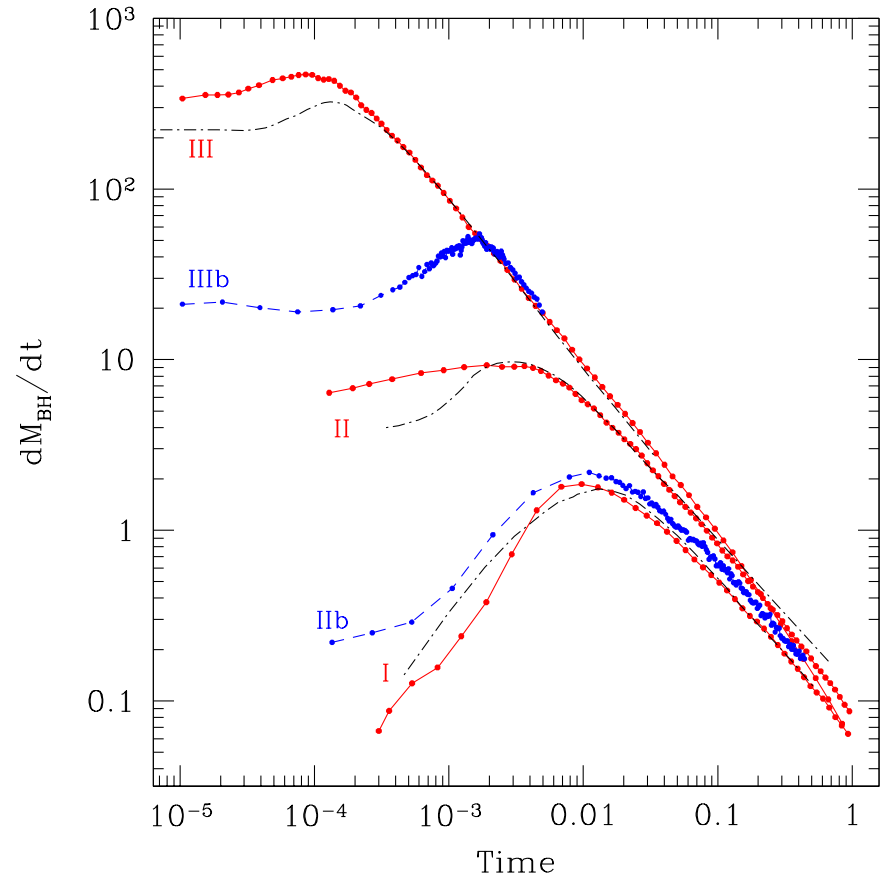

Fig. 12. Evolution of the growth rate of the central BH in clusters with initial conditions identical to models I, II and III of DS83. Dotdashed lines are from DS83. Model I does not include stellar collisions. Models II and III treat them as causing complete disruption of stars. Solid lines with dots are our results for these systems. Dashed lines with dots (labeled IIb and IIIb) show the effects of a realistic, SPH-generated, prescription for the outcome of collisions which allows partial disruptions and mergers (see text). We used 512000 to $2 \times 10^{6}$ super-stars in our simulations. " $N$-body" units are used. For models I and II, the time unit is $1.37 \times 10^{11} \mathrm{yrs}$ and the unit for $\mathrm{d} M / \mathrm{d} t$ is $2.6 \times 10^{-3} M_{\odot} \mathrm{yr}^{-1}$. In model III, these units are $9.81 \times 10^{11} \mathrm{yrs}$ and $5.8 \times 10^{-3} M_{\odot} \mathrm{yr}^{-1}$.

us. The most important effect of a realistic treatment of collisional outcome is a strongly reduced accretion rate. This is mainly due to the fact that most collisions are grazing and consequently produce low mass losses even for high relative velocities. Indeed, neglecting gravitational focusing, we get

$\frac{\mathrm{d} N_{\text {coll }}}{\mathrm{d} d_{\min }} \propto \frac{d_{\text {min }}}{R_{1}+R_{2}}$ for $d_{\text {min }}<R_{1}+R_{2}$

where $d_{\min }$ is the closest encounter distance for the equivalent 2 point-mass problem. The cumulative distribution of the fractional mass loss for model II is depicted in Fig. 13. Actually, the average mass loss per collision is as low as $0.08 M_{\odot}$ despite an average relative velocity for collisions of $v_{\text {rel }}=8.8 v_{*}$ (see Eq. (2)). These examples clearly demonstrate that any incorporation of collisions in galactic nuclei dynamics must account for partially disruptive events.

To conclude this series of tests, we turn to one of the most complete and widely used set of simulations of the longterm evolution of dense galactic nuclei published to date, namely the "direct" Fokker-Planck integrations by Murphy et al. (1991, hereafter MCD91). These authors included the following physics in their computations:

- 2-body relaxation. It is treated in the standard FokkerPlanck way (for a description of the multi-mass FP scheme

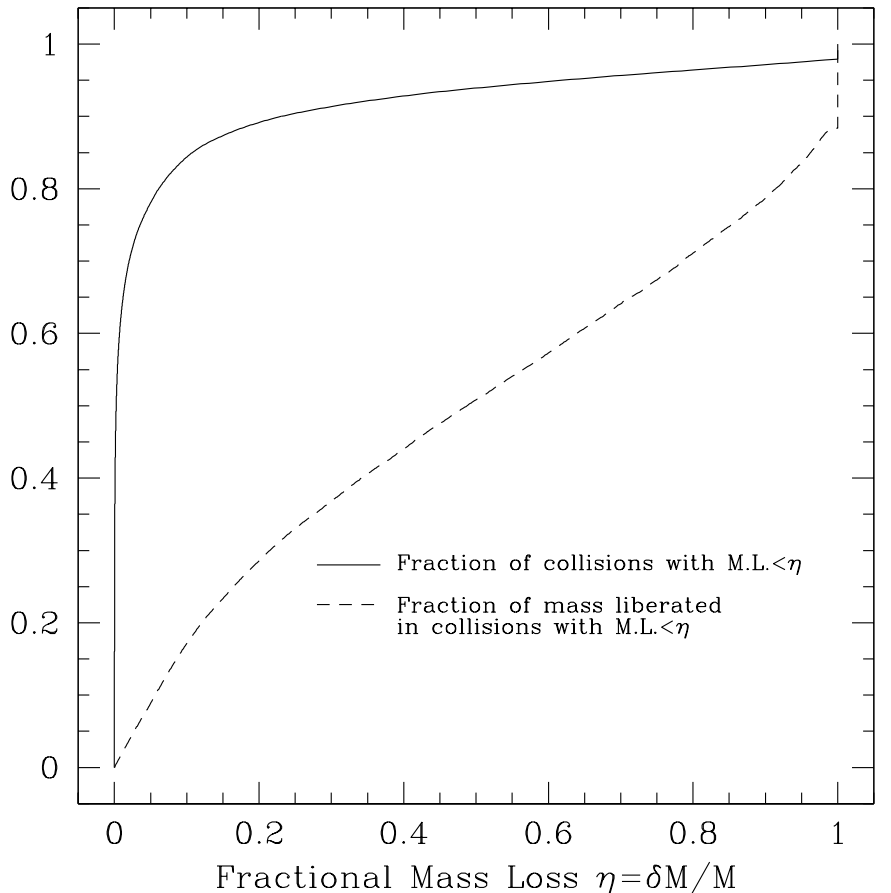

Fig. 13. Cumulative distribution of the fractional mass losses in collisions for a simulation of model II with improved treatment of collisions (see text). All collisions occurring before time $T=0.1 \tilde{\mathcal{U}}_{\mathrm{t}}=$ $1.37 \times 10^{10} \mathrm{yrs}$ are included in this count. The solid line shows the number fraction of all collisions which resulted in a fractional mass loss lower than a given amount $\eta$. The dashed line indicates what mass fraction of collisionally released gas came from collisions with fractional mass loss lower than $\eta$.

see, e.g. Murphy \& Cohn 1988 and references therein). Note that, in the FP scheme, the cluster is represented as a set of DFs, each of which represent a discretized mass class, i.e., stars that have all the same stellar mass.

- Stellar collisions. To get the mass loss for individual collisions, MCD91 use a semi-analytical method derived from the procedure invented by Spitzer \& Saslaw (1966). It works by decomposing the stars into thin columns of gas parallel to the relative velocity and imposing conservation of momentum for each, completely inelastic, collision between a column from one star and the corresponding column of the other star. No lateral mass, energy or momentum transport is considered. The MS stars are assumed to be $n=3$ polytropes with $M_{*} \propto R_{*}$. These mass-loss rates are then averaged over impact parameter and relative velocities to get rates that depend only on velocity dispersion and mass ratio which allows the authors to compute the instantaneous mass-loss rate for any mass class, due to collisions with stars from any other (or same) mass class. The total mass loss for a given time step and mass class is then converted into a number of stars to be removed from the class. This is obviously quite an inaccurate representation of the real way collisions change the masses of individual stars. Mergers are not included in this formalism.

- Tidal disruptions. Stars that get closer to the BH than the tidal disruption radius are assumed to be completely 
disrupted and their mass is instantaneously and fully accreted by the $\mathrm{BH}$. Although our numerical scheme is widely different, we use basically the same assumptions, here. Hence, we refer to MCD91 and Cohn \& Kulsrud (1978) for a description of how this is implemented in FP codes.

- Stellar evolution. A simple prescription is used in which stars stay on the MS for $T_{\mathrm{MS}}\left(M_{*}\right)$ and then turn abruptly into compact remnants (CR). No giant phase is simulated and all mass loss occurs at the end of the MS. See MCD91 for the specification of $T_{\mathrm{MS}}\left(M_{*}\right)$ and the $\mathrm{MS} \rightarrow \mathrm{CR}$ relation.

The initial stellar clusters are Plummer models with a core radius of $1 \mathrm{pc}$. The total stellar mass is initially $8.291 \times$ $\left(10^{9}, 10^{8}, 10^{7}, 10^{6}\right) M_{\odot}$ for models of classes " 1 ", " 2 ", “ 3 " and "4", respectively. The stars are initially on the MS and obey a power-law mass spectrum, $\mathrm{d} N_{*} / \mathrm{d} M_{*} \propto M_{*}^{-\alpha}$ between 0.3 and $30 M_{\odot}$, with $\alpha=1.5,2.5$ and 3.5 for cases "A", "B", "C".

The cluster is seeded with a $\mathrm{BH}$ of mass $M_{\mathrm{BH}}=10^{4} M_{\odot}$ at its center. The $\mathrm{BH}$ eventually swallows all the gas lost by stars, through normal evolution, collisions or tidal disruptions, but its growth rate is limited by the Eddington rate $\dot{M}_{\mathrm{E}}=L_{\mathrm{E}} /\left(\eta c^{2}\right)=4 \pi G \mu_{\mathrm{e}} M_{\mathrm{BH}} m_{\mathrm{p}} /\left(\eta c \sigma_{\mathrm{T}}\right) \simeq 2.5 \times$ $10^{-2} M_{\odot} \mathrm{yr}^{-1}(\eta / 0.1)^{-1}\left(M_{\mathrm{BH}} / 10^{6} M_{\odot}\right)$ where $\eta$ is the efficiency factor for conversion of mass into radiation during the accretion process, $\mu_{\mathrm{e}}$ is the molecular weight per free electron of the accreted gas $\left(\simeq 1.13\right.$ for solar composition), $m_{\mathrm{p}}$ the mass of the proton and $\sigma_{\mathrm{T}}$ Thomson's cross-section. A "standard" value of $\eta=0.1$ is used. If the instantaneous rate of gas production from the stars, $\dot{M}_{\text {prod }}$, exceeds $\dot{M}_{\mathrm{E}}$, only an amount $\dot{M}_{\mathrm{E}}$ accretes on the $\mathrm{BH}$ while the remaining accumulates into a central "reservoir" - presumably an accretion disk - to be accreted later when $\dot{M}_{\text {prod }}$ has declined below $\dot{M}_{\mathrm{E}}$. The gas is assumed to be funneled completely and instantaneously to the center, i.e. no gas remains in the stellar cluster or is expelled from the nucleus. The structure of this reservoir is not resolved in the simulations. Instead, it is assumed to be small enough to contribute to the potential as a central point mass, exactly as the BH. However, distributing the central mass in two components, the BH and this reservoir, can still influence the dynamics slightly through the fact that only the mass of the $\mathrm{BH}$ is used to compute the tidal disruption radius. On the other hand, interactions between the gas reservoir and stars are neglected (see Sect. 6.2).

We have simulated all models specified by MCD91 with 256000 super-stars. For models of class B, we have redone the simulations with $10^{6}$ super-stars. We basically mimic the initial conditions and physics of MCD91. For instance, we used $\gamma=0.4$ for the Coulomb logarithm. Note that MCD91's FP method imposes an isotropic velocity distribution while our code allows anisotropy to develop. In addition to the obvious differences imposed by the use of a very different simulation algorithm, the following distinctions in the treatment of the physics have to be noted:

- The collisions are treated much more realistically, on a particle-particle basis and outcomes are given by our SPHgenerated grid for which realistic stellar structures have been used. The collisional modification of orbits is accounted for and mergers may occur;

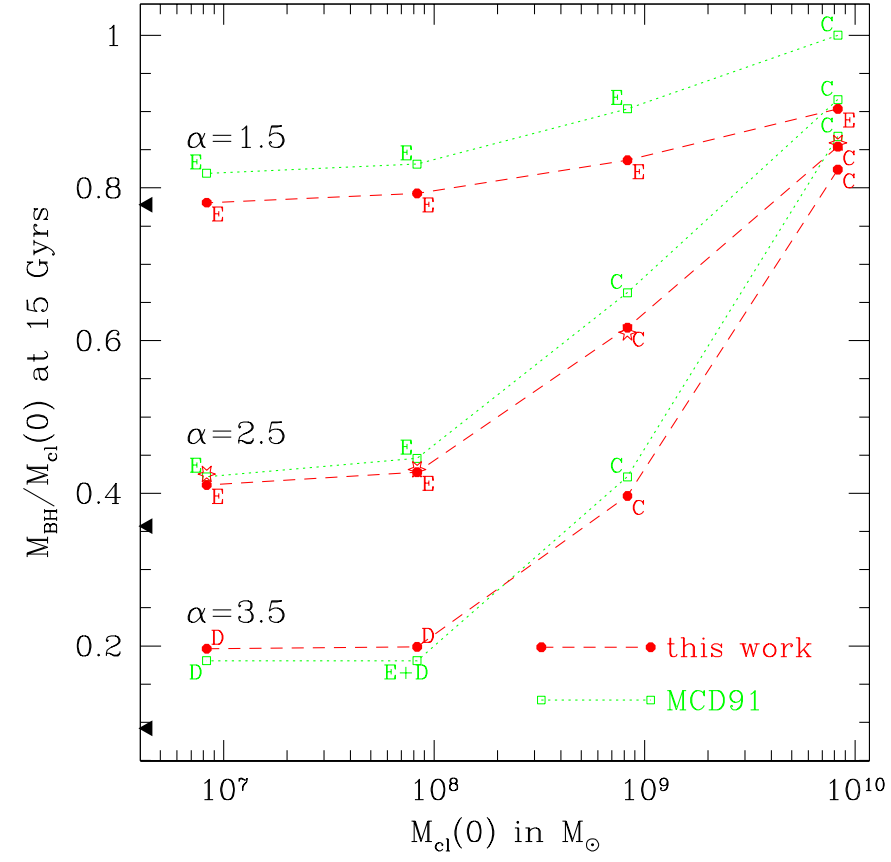

Fig. 14. Final BH mass for all the MCD91-like models. The lines connect models with the same IMF slope. We compare our results (dashed lines) to those from MCD91 (dotted lines). Solid dots are for simulations with 256000 super-stars; the open star symbols are for B models with $10^{6}$ super-stars. The triangles on the left axis indicate the total fractional mass loss due to stellar evolution for IMF with $\alpha=1.5,2.5,3.5$, at an age of $15 \mathrm{Gyrs}$. This corresponds to the final BH's mass expected if stellar evolution was the only feeding process and no star could escape the nucleus. Letters indicate the process whose contribution to the final BH's mass dominates: "E" stands for stellar evolution, "C" for collisions and "D" for tidal disruptions. Most of the discrepancies between our results and those of MCD91 is due to the lower contribution of collisions (see text).

- The stellar evolution is slightly different from MCD91 (see Sect. 4.1);

- A "continuous" mass spectrum is used instead of the discrete mass classes of MCD91. To get the same average stellar mass as these authors, the mass range is extended to $0.258-34.8 M_{\odot}$. Also, masses as low as $0.01 M_{\odot}$ may be produced in collisions (smaller collisional products are not allowed) while MCD91 use a "hard", constant minimum of $0.3 M_{\odot}$;

- We use a $M_{*}-R_{*}$ relation from MS stellar models (Schaller et al. 1992; Meynet et al. 1994; Charbonnel et al. 1999; Chabrier \& Baraffe 2000) to determine collisional crosssections and tidal disruption radii;

- Stellar evaporation, due to gradual energy gain through 2body relaxation (see Paper I), is allowed in our models but MCD91 apparently enforce evolution at constant total mass which seems reasonable because, for a cluster with no tidal truncation, diffusive relaxation is expected to be inefficient. Indeed, it takes longer and longer to increase the (negative) energy of a star toward $E>0$, as it stays for a larger and larger fraction of its orbital time in large-radius, low-density regions where relaxation is vanishingly small 
(a)

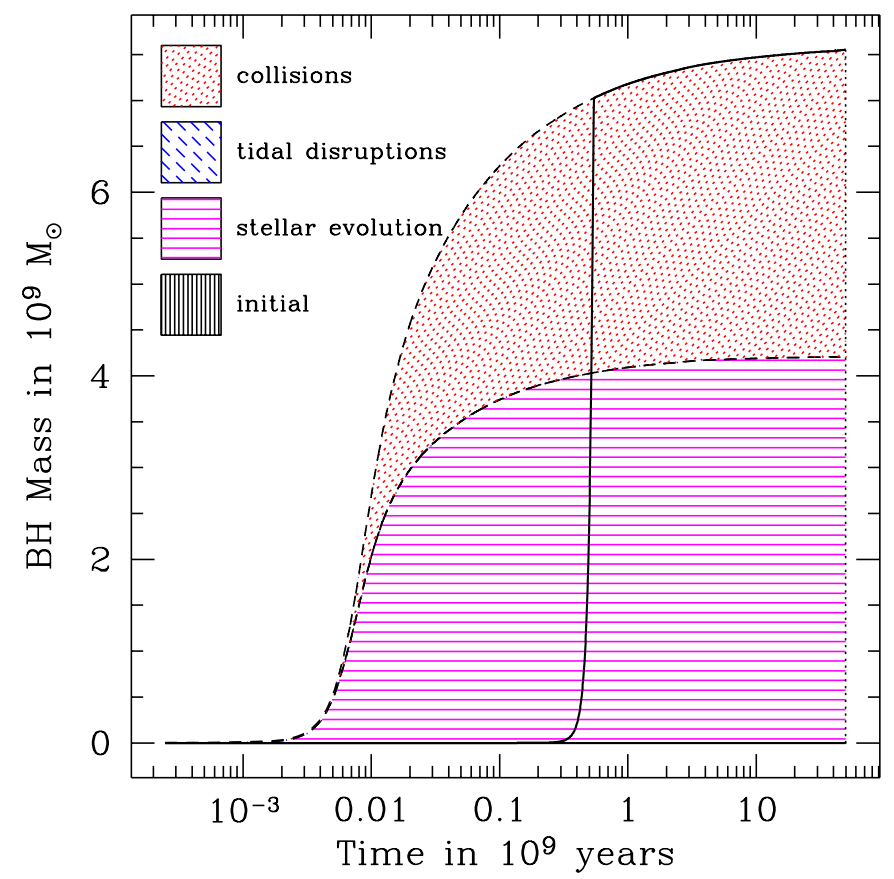

(c)

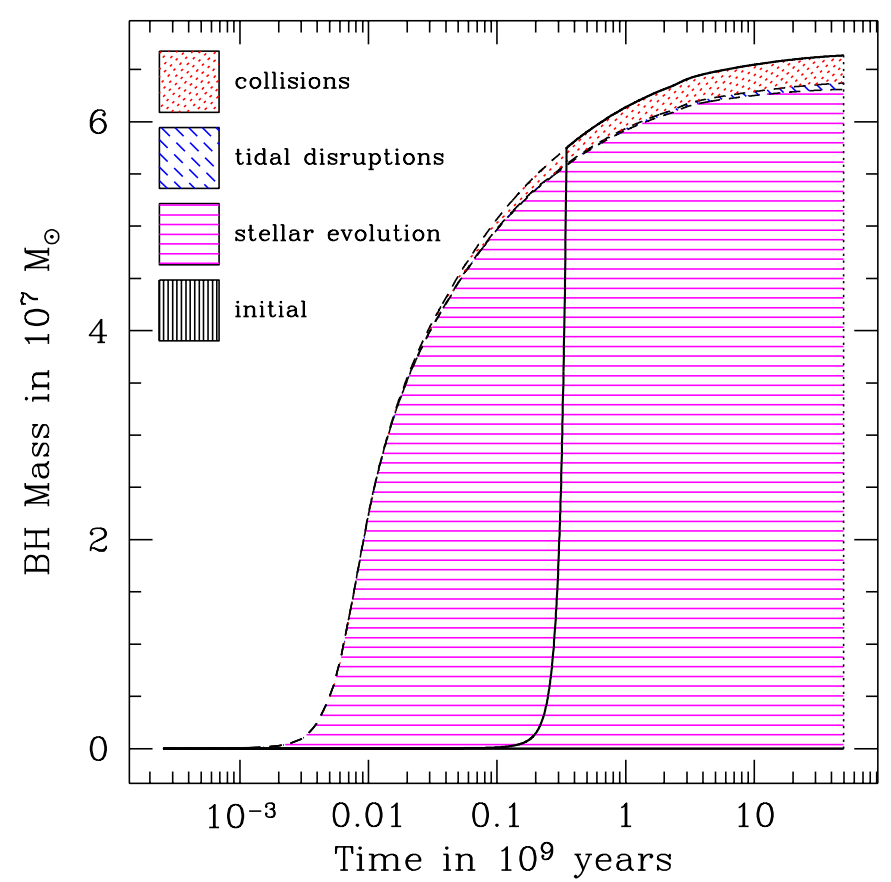

(b)

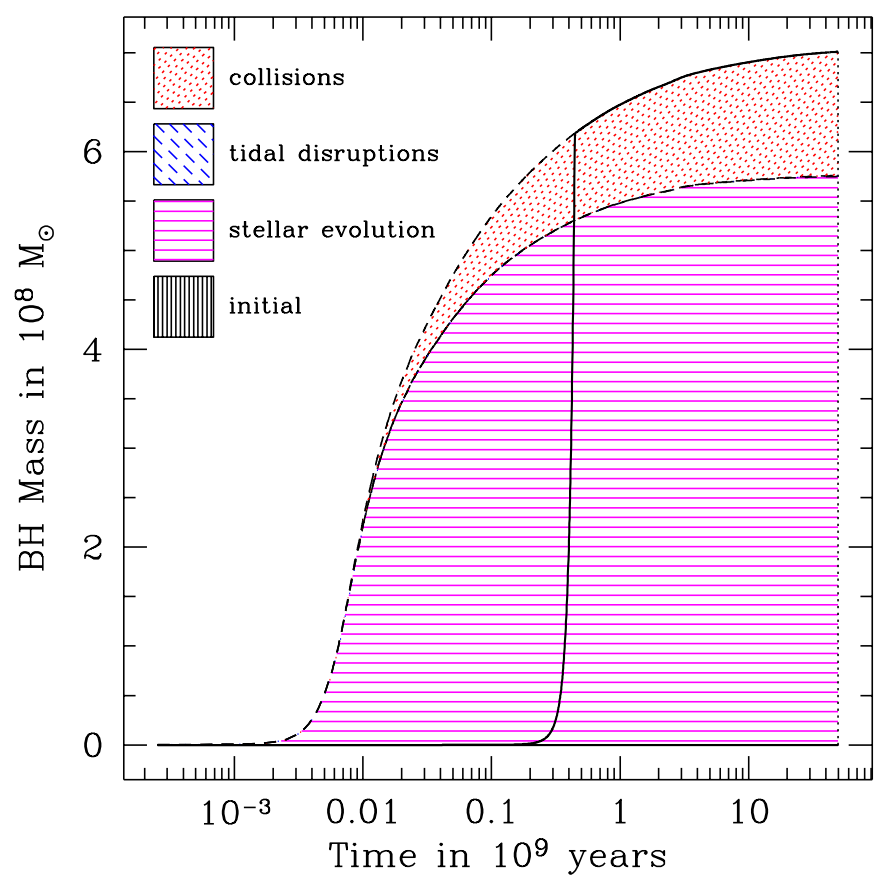

(d)

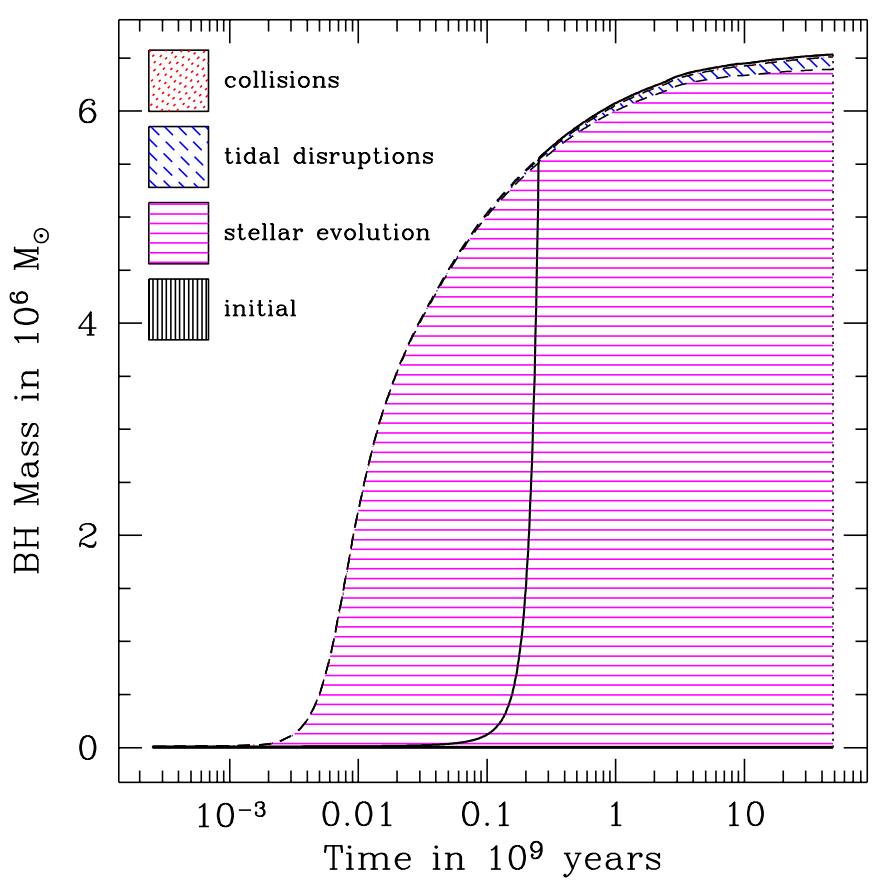

Fig. 15. Evolution of the central mass ( $\mathrm{BH}+$ gas reservoir) for MCD91-like models of class $\mathrm{A}(\alpha=1.5$ for the IMF). The various hatching styles indicate the origin of the gas. The initial BH mass is too small to be visible on these diagrams (dark gray hatching). The thick line is the mass of the central BH, as limited by Eddington luminosity. Our simulations were realized with 256000 super-stars. Note that the ordinate mass units are different in each panel. For this top-heavy stellar spectrum, the role of stellar evolution is clearly dominant even in model A where the high stellar density boosts the collision rate. Panels a) to d) correspond to decreasing initial cluster mass (see text). 
(a)

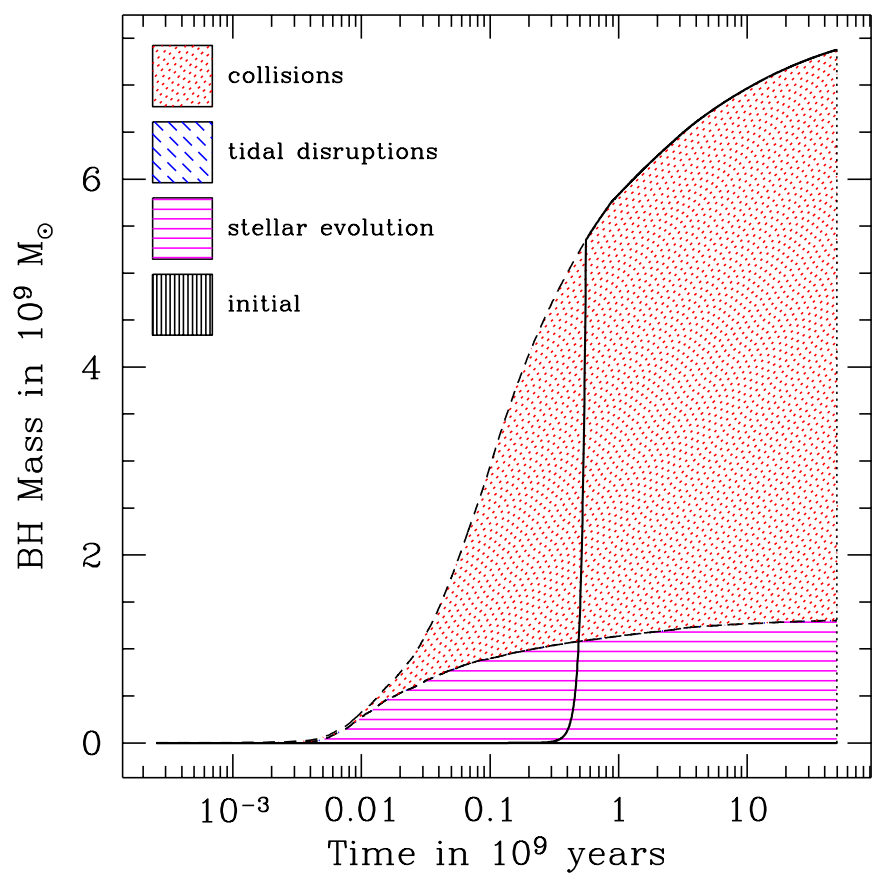

(c)

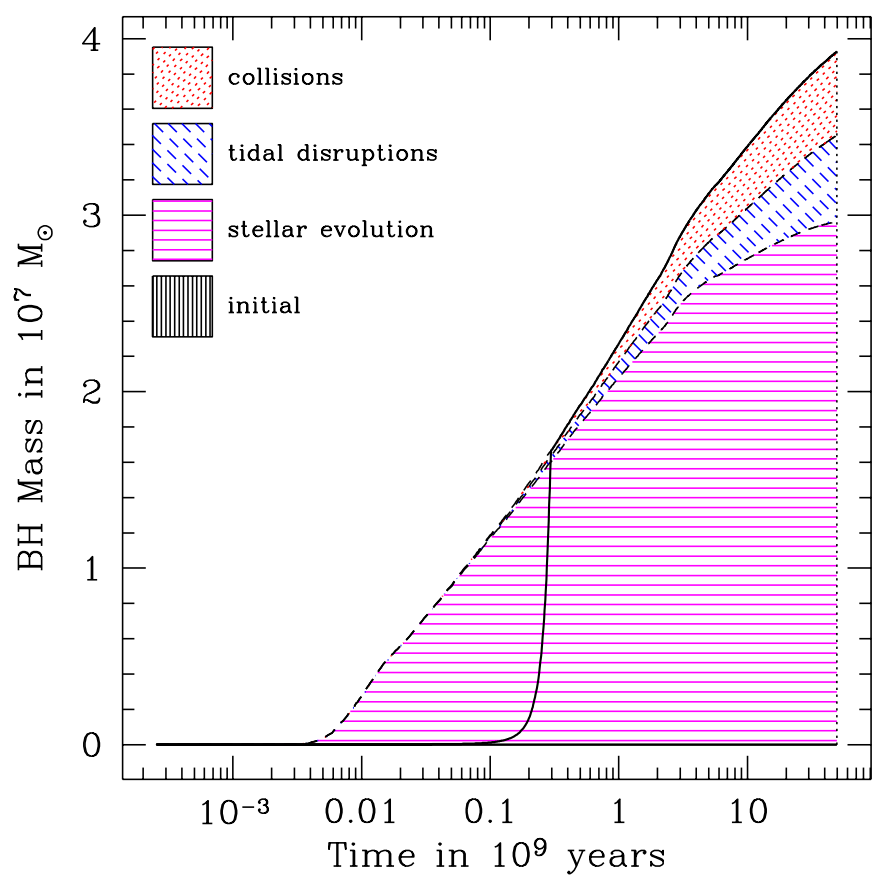

(b)

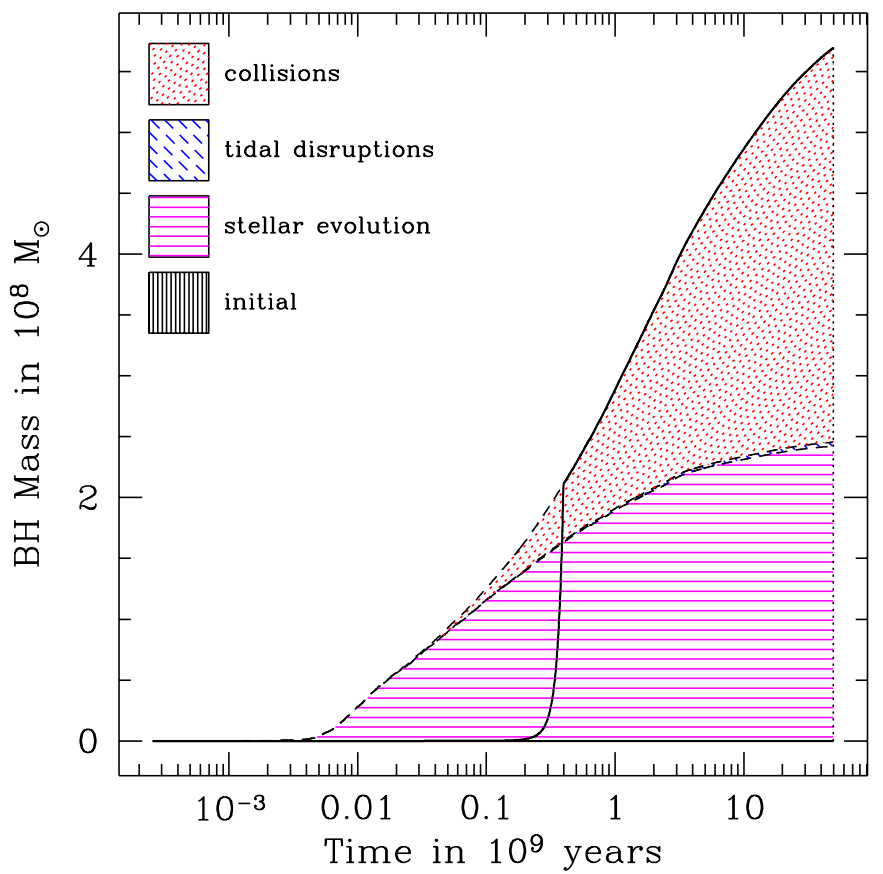

(d)

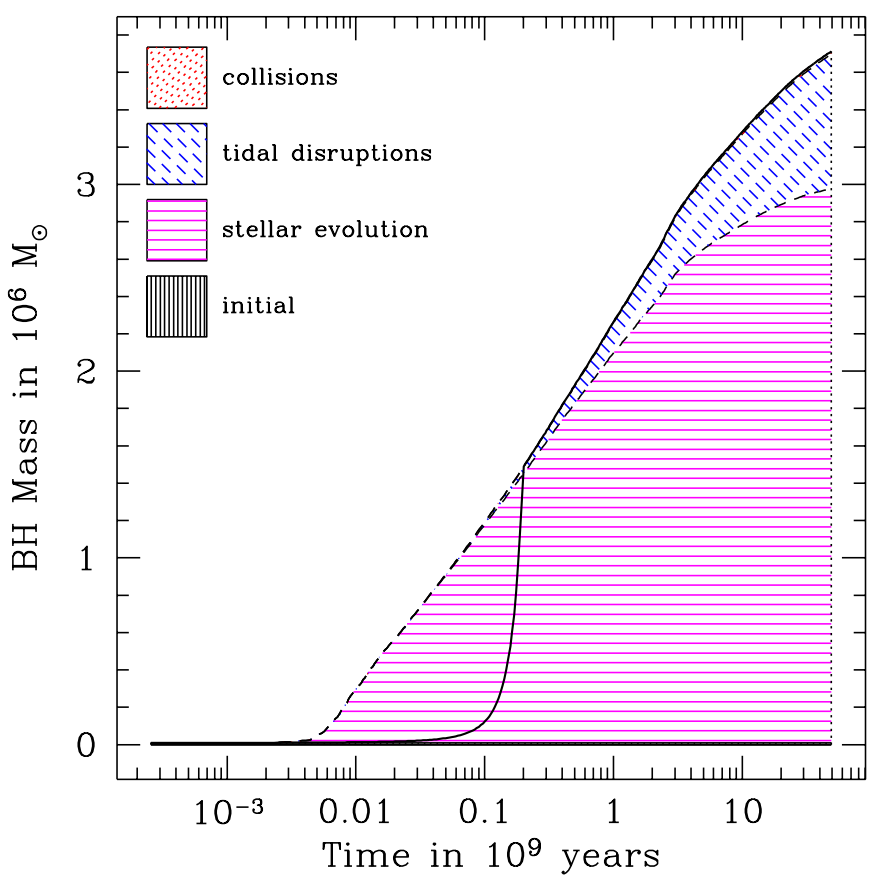

Fig. 16. Same as Fig. 15, but for models of class B $(\alpha=2.5)$. 
(a)

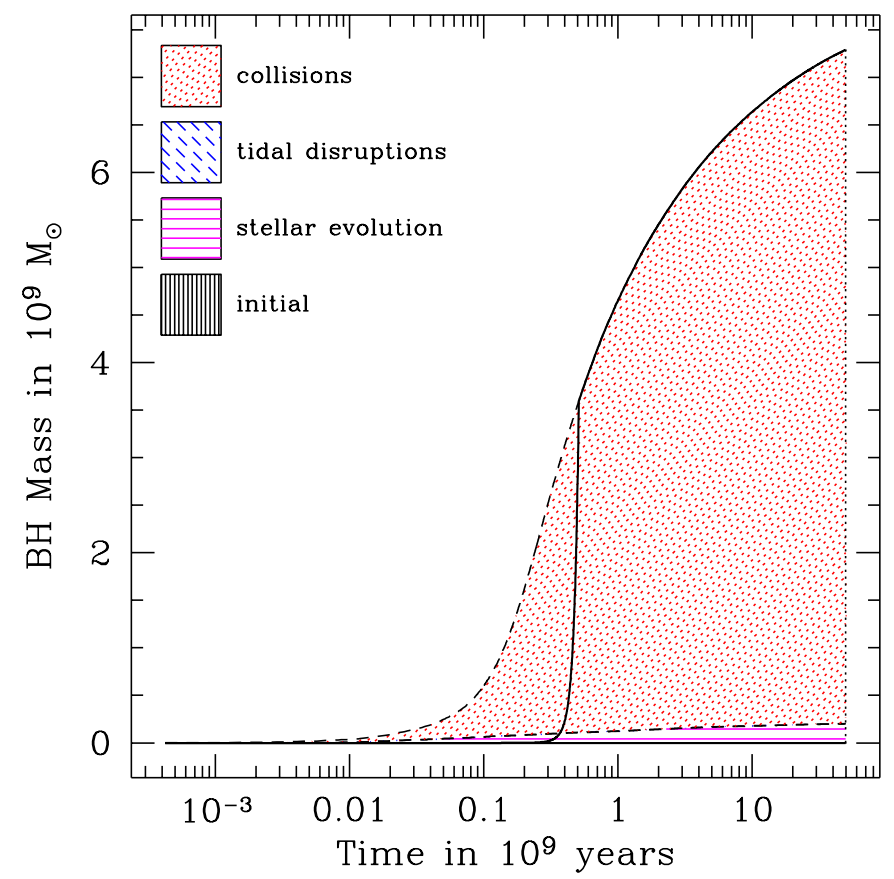

(c)

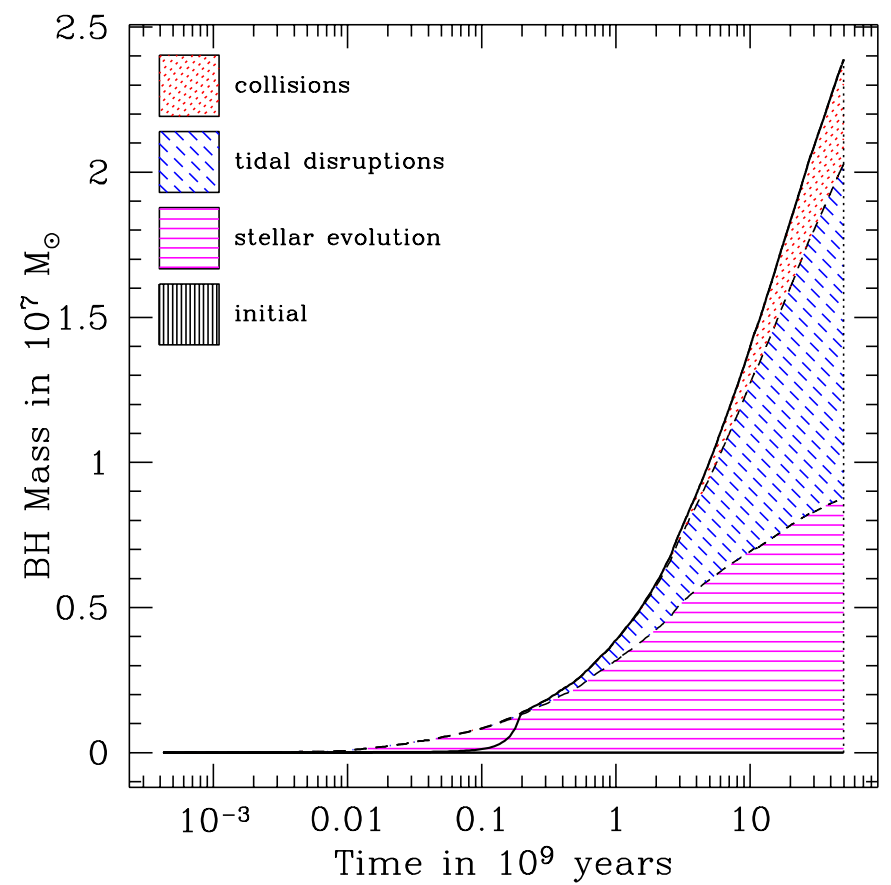

(b)

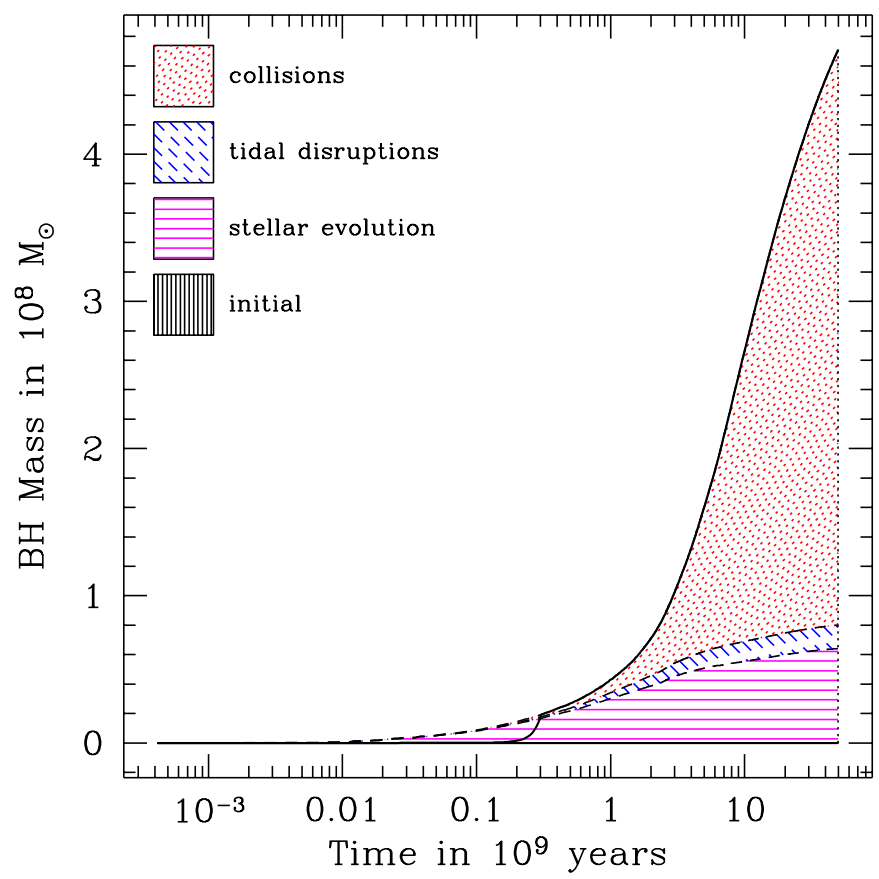

(d)

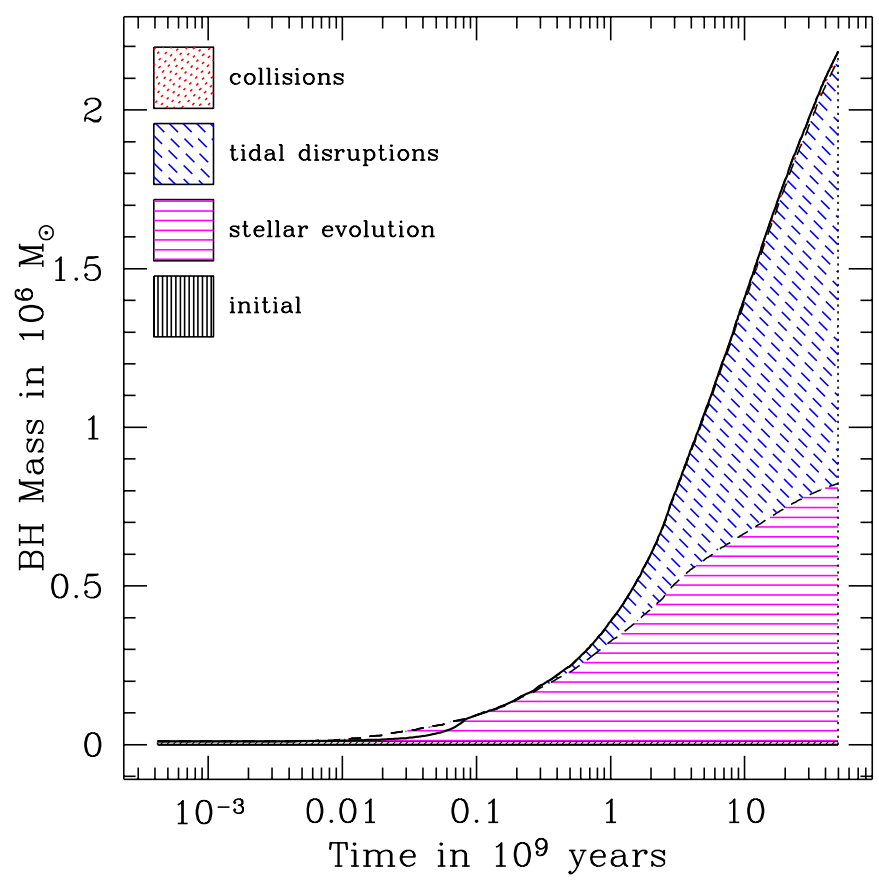

Fig. 17. Same as Figs. 15 and 16, but for models of class C $(\alpha=3.5)$. In this model with a stellar IMF strongly dominated by low masses, the role of stellar evolution is minimized so that collisions and tidal disruptions dominate the gas production rate. 
(a)

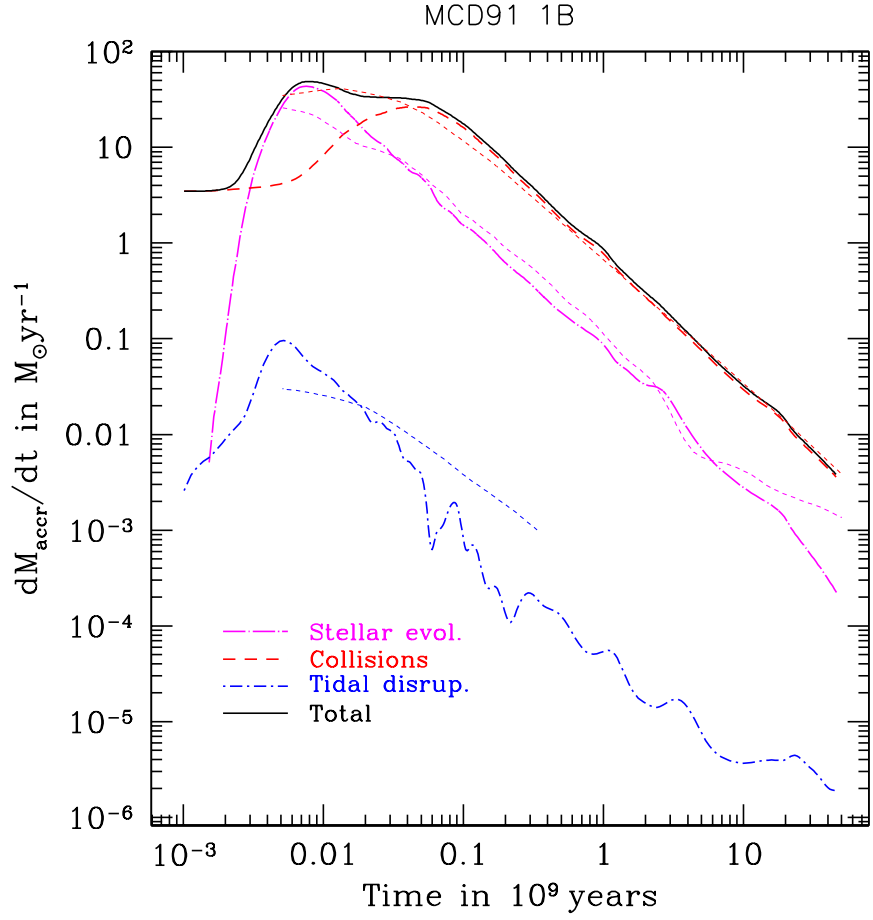

(c) (b)

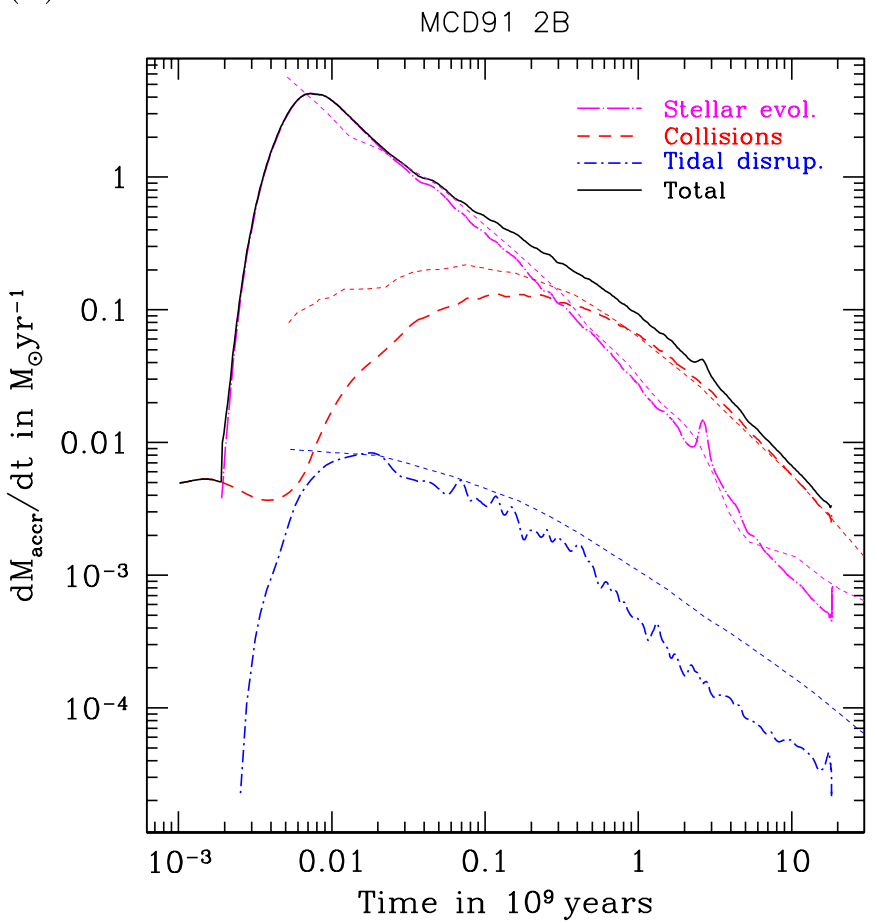

(d)

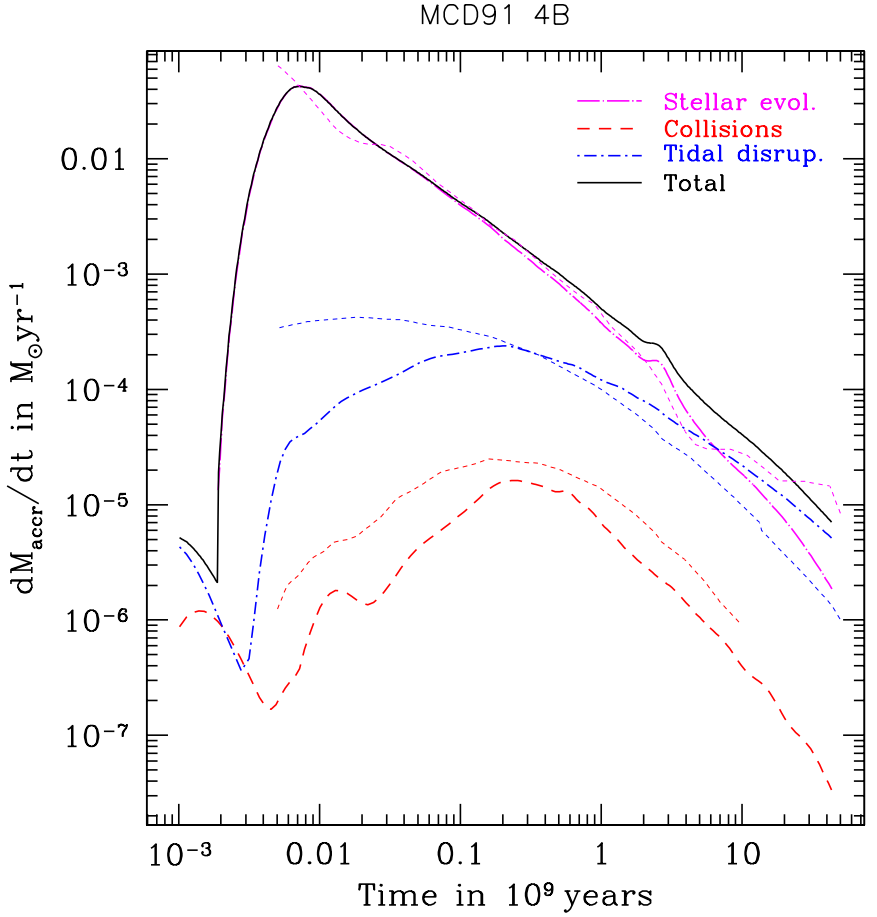

Fig. 18. Evolution of the gas production rate for galactic nucleus models with initial conditions corresponding to models $1 \mathrm{~B}-4 \mathrm{~B}$ of MCD91. We plot the amount of gas the stars release per year through different channels: stellar evolution, collisions and tidal disruptions. Note that, at early times, only a fraction of this gas is accreted by the central BH while the remaining accumulates in some central reservoir. The thin dotted lines are the results of MCD91 but, for clarity, their total rates are omitted. Our simulations were realized with $10^{6}$ super-stars. The small-scale oscillations present in our curves are numerical noise. See text for further comments. 
(a)

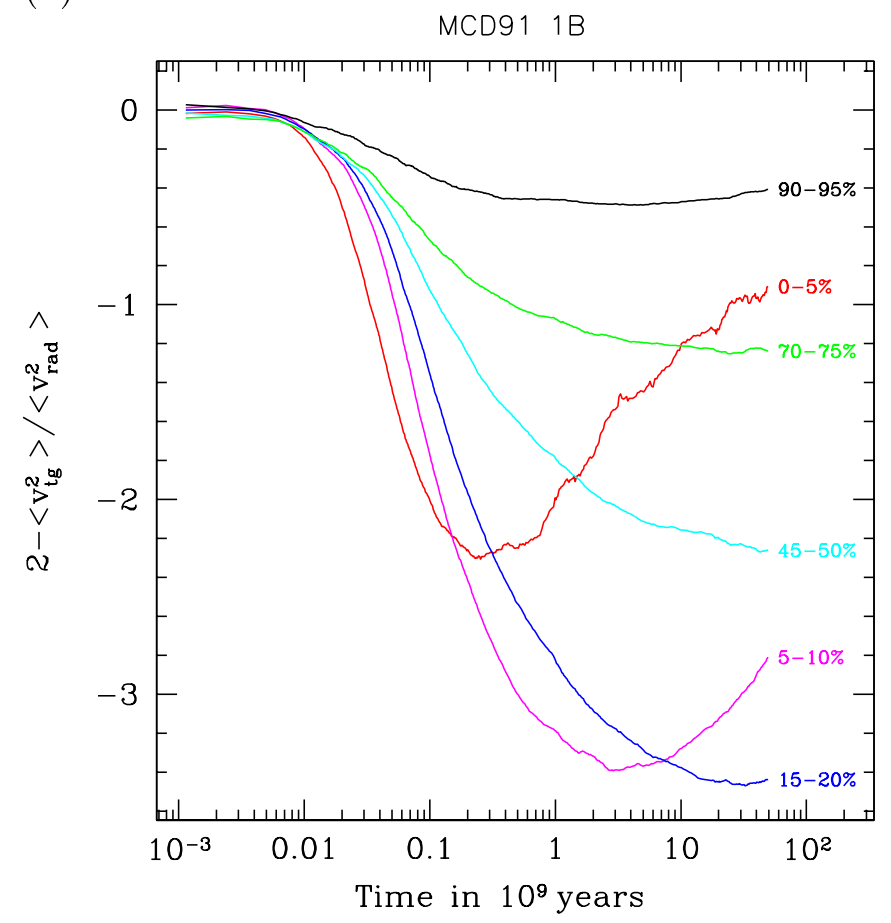

(b)

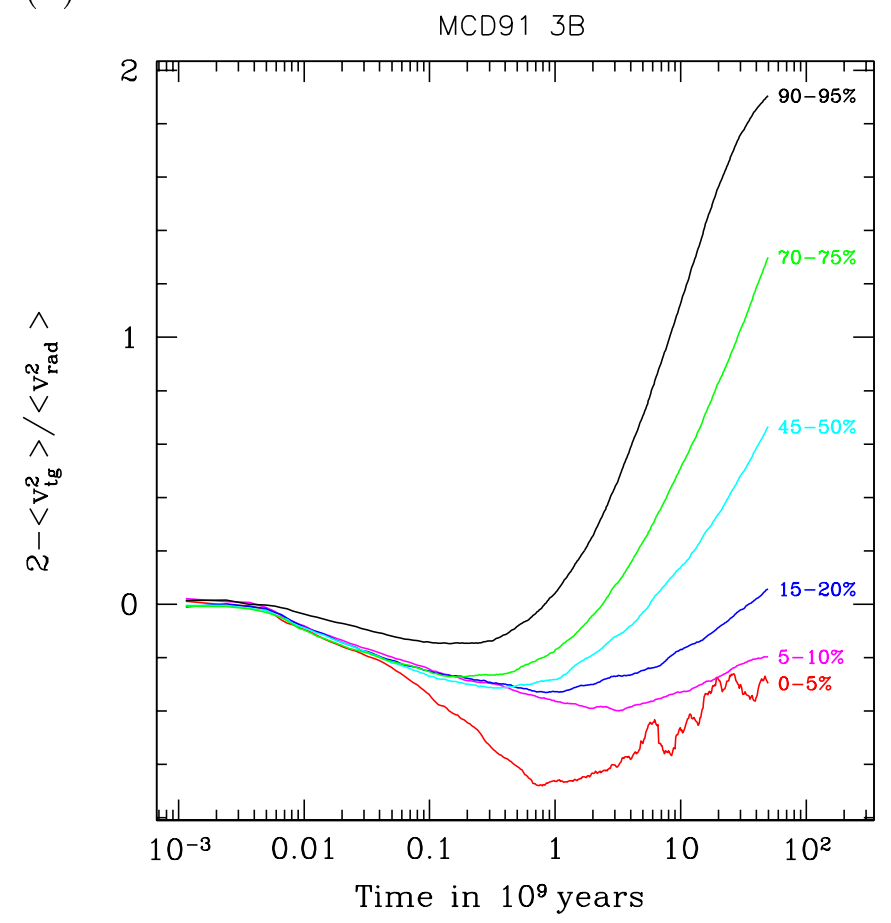

Fig. 19. Evolution of the anisotropy for 2 models of class B simulated with $10^{6}$ super-stars. We show the anisotropy parameter averaged over Lagrangian shells bracketed by the indicated fraction of the (remaining) stellar mass. Note how strong a tangential anisotropy develops in model $1 \mathrm{~B}$, certainly in response to the adiabatic growth of the central BH. At later time, relaxation cause the central parts to slowly return to a more isotropic velocity distribution. The evolution of anisotropy in the lighter model 2B is strikingly different. For clarity, the curves have been slightly smoothed.
(Hénon 1960, 1969). For B models with $10^{6}$ super-stars, we tried to forbid relaxation-driven stellar evaporation by discarding "super-encounters" that lead either super-star to escape the system. The results appear not to be significantly altered by this special treatment.

Among the results published by MCD91, those with which comparisons are most easily carried out and which are of prime interest for us, concern the growth of the central $\mathrm{BH}$ and how various processes contribute to it. In Fig. 14, for all 12 models considered by MCD91, we compare the final mass of the central BH and indicate which process contributed most to this mass. We confirm that, unless the stellar mass spectrum is strongly bottom-heavy (case $\mathrm{C}, \alpha=3.5$ ) low-mass models are dominated by stellar evolution. $\mathrm{C}$ models of low mass are the only ones for which tidal disruptions are a significant fuel source. At higher (initial) stellar densities, collisions dominate, with the densest A model as an exception. The main source of discrepancy between our findings and those of MCD91 is the more minor role of collisions in our simulations. While it is difficult to evaluate how MCD91's use of fixed classes of $M_{*}$ translate in their collisional gas production rate, it is certain that their $n=3$ polytropes models experience more mass loss in off-center collisions than more realistic stars (Freitag \& Benz $2002 \mathrm{~b}$, a) and that their mass-radius relation lead to an overall overestimate of collision cross-section. A secondary source of mismatch is our different prescription for stellar evolution. The temporal evolution of the central mass $(\mathrm{BH}+$ gas reservoir) for all 12 models is depicted in Figs. 15 to 17.

A more detailed comparison is realized for models of class B for which MCD91 published the curves of the rate of gas production through each process. Our results are reported in Fig. 18. Here again, we notice that the main difference with MCD91 is that their collisional rate is much higher at early times. This is probably due to the presence of massive stars for which their assumptions about stellar structure and radius should lead to the most severe overestimate of collisional massloss. In fact, in regard of how different (and more detailed) our treatment of collisions is, it is very surprising how similar the collisional gas production rates are at late times. The tidal disruption rates are very similar at early time, with the exception of the least dense model (4B). At later times, our tidal gas production rate decreases at a steeper rate (as compared to MCD91) for the two densest models, while the contrary is true for models $3 \mathrm{~B}$ and $4 \mathrm{~B}$. One possible explanation for the lowest late-time rates in dense models is that significant tangential anisotropy develops in the central parts of these clusters, probably in response to the rapid and, hence, nearly adiabatic, growth of the $\mathrm{BH}$, a process which does not significantly affect the "light" clusters. This aspect is illustrated in Fig. 19. Obviously, stars on low eccentricity orbits are less likely to enter the loss cone, an aspect of the dynamics that MCD91 could not simulate with their isotropic code. On the other hand, this does not explain why we get a higher late time disruption rate for the lower density clusters. 


\section{Conclusions}

\subsection{Summary}

In this second paper about our Monte Carlo code for star cluster simulations, we have described our inclusion of physical processes pertaining to the dynamics of galactic nuclei.

Taking advantage of the particle-based approach of the MC code, collisions between MS stars are treated with a high level of realism. The MC sampling reproduce the rate of collisions between stars of various masses and the distribution of relative velocities (and impact parameters) in a straightforward way. The outcome of collisions are obtained by interpolation into a comprehensive database of results from SPH simulations (Freitag \& Benz 2002b,a). This is an important improvement over previous works that included the role of collisions in the dynamical evolution of galactic nuclei but relied on simpleminded prescriptions for the results of collisions. In the past, only Rauch (1999) has attempted to use the outcome of a limited number of SPH simulations by M. Davies to find fitting formulae for their outcome and incorporate collisions in cluster models. It is, however, doubtful that these results, obtained with polytropic stellar models and from a relatively small domain of the parameter space can be applied for realistic stars and other relative velocities and/or impact parameters (Freitag \& Benz 2002b).

The second important feature of the dynamics of a galactic nucleus, as compared to a globular cluster, is the likely presence of central BH with a mass in excess of $10^{6} M_{\odot}$ (although some globular clusters, like M 15, may harbor a central BH, see Gebhardt et al. 2000 and van der Marel 2001). In our code, we assume the BH stays perfectly at the center (see below) and treat its contribution to the potential as that of a Newtonian point mass. The neglect of relativistic effects on stellar orbits is probably a good approximation, according to Rauch (1999) who concluded that they seem to have no noticeable influence in his simulations. The $\mathrm{BH}$ grows by accreting gas released by the stellar system through stellar evolution, collisions and tidal disruptions. Whole stars may also be swallowed if they directly plunge through the horizon. This latter process completely supersedes tidal disruption for MBH more massive than a few $10^{8} M_{\odot}$ because, then, the tidal disruption radius is formally inside the horizon. For the time being, the process of tidal disruption itself is treated as simply as possible, by assuming complete disruption of every star that enters the Roche zone around the $\mathrm{BH}$. On the other hand, we test for super-stars entering the so-called "loss-cone", i.e. getting onto disruption orbits, in a detailed way by simulating the fine-grained diffusion caused by relaxation on the direction of a super-star's velocity.

Other improvements include a simple treatment of stellar evolution which assumes that stars transform directly from MS to compact remnants, in a similar spirit to what has been done by previous investigators (Norman \& Scoville 1988; Murphy et al. 1991). Also, we have implemented "particle doubling" to maintain high resolution even in simulations where a lot of stars are either destroyed or ejected from the cluster.

These new features have been extensively checked against (semi-)analytical predictions and simulations from the litera- ture. In most cases, the tests are highly successful. In particular, collision rates are nicely reproduced, not only when integrated over the whole cluster but also as a function of distance from the center and of the masses of stars. The effects on the stellar cluster of an adiabatically growing central black hole are nearly perfectly in agreement with theoretical predictions. The standard "Bahcall and Wolf" $R^{-7 / 4}$ density cusp is obtained in the case tidal disruptions are taken into account but collisions are switched off or inefficient. In highly collisional models, a shallower cusp, with exponent around -0.5 is produced, in good agreement with what was reported in previous studies. Gas production by the stellar cluster through various processes (tidal disruptions, collisions, stellar evolutions) are also in good agreement with results from the literature, obtained with a variety of numerical methods. Most of the discrepancies can be easily explained. In particular, it appears that the role of collisions has been overestimated in previous works, due to over-simplified assumptions about the collisional outcome (complete disruptions or simple semi-analytical treatment applied to polytropic models) and, maybe, to their being included into the simulations in a quite nonphysical way, in the case of direct Fokker-Planck methods. Concerning tidal disruptions, some disagreement, for which we have found no straightforward explanation, is observed with the works of Duncan \& Shapiro (1982) and Amaro-Seoane \& Spurzem (2001). These mismatches are not severe, however, and, as the resolution of the simulations by Duncan \& Shapiro (1982) was quite low ${ }^{13}$ and the results plotted by Amaro-Seoane \& Spurzem (2001) come only from preliminary computations, we can not draw definitive conclusions from these comparisons. Furthermore, there is no clear trend in these differences and we get better agreements in other cases (with, e.g., model I of Duncan \& Shapiro 1983), a fact which seems to exclude any important flaw in our algorithm. Unfortunately, $N$-body methods seem still a long way from allowing simulations of the relaxational dynamics around a black hole and, thus, providing more direct check of our approach and, more generally, of the applicability of the loss-cone theory and the Chandrasekhar treatment of relaxation in such a situation (e.g., Spurzem \& Kugel 2000).

\subsection{Future work}

In Sect. 8.2 of Paper I, we have already mentioned many improvements/additions that we plan to incorporate in future versions of the code. Here, we update and complete this list:

1. Capture of compact stars by the central BH through emission of gravitational radiation. This process has been presented in Sect. 1.1. Predicting the rate and characteristics of these events has recently become a main focus of our work and very encouraging results have already been reported in Freitag (2001).

2. Refined treatment of stellar evolution. The most severe shortcoming of our present modeling of SE is the absence

\footnotetext{
13 They used a few thousands particles but their cloning algorithm increased the relative resolution at large negative energies, i.e. close to the $\mathrm{BH}$.
} 
of giant phase. Taking it into account should greatly enhance the number rates of collisions and tidal stripping (Davies et al. 1998; Bailey \& Davies 1999; Magorrian \& Tremaine 1999; Syer \& Ulmer 1999) although the amount of released gas may be limited due to the very low density of giants' envelopes and this may not increase the BH's growth as this gas would be liberated anyway through stellar evolution. Others aspects of SE that we shall incorporate are: progressive mass loss on the MS, natal kicks for neutron stars and collisional rejuvenation.

3. Refined treatment of tidal interactions. We should treat the hydrodynamical nature tidal disruptions with the same level of realism that we achieved for collisions. This will be essential if we want to cope with envelope-stripping of giant stars (Di Stefano et al. 2001) and other "tidally perturbed" stars (Alexander \& Livio 2001). Stars can also be tidally captured by the central BH. As more and more orbital energy is transfered to oscillations at each subsequent pericenter passage, disruption is the most probable outcome (Novikov et al. 1992).

Assuming that the $\mathrm{BH}$ stays fixed at the center of the cluster is an over-simplification. If the central BH's wandering is of larger extent than its tidal disruption radius $R_{\text {disr }}$, there will be no regime of empty loss cone (Sigurdsson \& Rees 1997). For a cluster with core radius $R_{\mathrm{c}}$, equipartition predicts a wandering radius of order $R_{\mathrm{w}} \approx R_{\mathrm{c}} \sqrt{M_{*} / M_{\mathrm{BH}}}$ (Bahcall \& Wolf 1976; Lin \& Tremaine 1980; Chatterjee et al. 2002), and

$$
\frac{R_{\mathrm{w}}}{R_{\mathrm{disr}}} \approx 400\left(\frac{R_{\mathrm{c}}}{1 \mathrm{pc}}\right)\left(\frac{R_{*}}{R_{\odot}}\right)^{-1}\left(\frac{M_{*}}{M_{\odot}}\right)^{\frac{5}{6}}\left(\frac{M_{\mathrm{BH}}}{10^{6} M_{\odot}}\right)^{-\frac{5}{6}} .
$$

See Magorrian \& Tremaine (1999) for hints at the possible effects of the wandering on the tidal disruption rate. Young (1977) made a rough estimate of the correction and deemed it not to alter the disruption rate drastically. However, as suggested by Alexander \& Livio (2001), these motions of the $\mathrm{BH}$ may allow stars that have been tidally perturbed to escape further, disruptive, close interactions with the $\mathrm{BH}$, which is of high potential interest for the Galactic center.

4. Large angle scatterings. 2-body gravitational encounters with impact parameter of order or smaller than $b_{0}=G\left(M_{1}+\right.$ $\left.M_{2}\right) / V_{\text {rel }}^{2}$ lead to scattering angles of order $\pi$. Although they only contribute a fraction $\ln \left(b_{\max } / b_{0}\right)^{-1}<0.1$ to the overall relaxation (Hénon 1973, p. 198), they may dominate the rate of evaporation from the cusp (Lin \& Tremaine 1980; Goodman 1983) and of captures on relativistic orbits (Sigurdsson \& Rees 1997). Such "kicks" can not be decomposed into smaller deflections but can probably be introduced explicitly in the MC code in a similar way as collisions.

5. Inclusion of binary stars. In a "normal" population (Duquennoy \& Mayor 1991), most binaries have (internal) orbital velocities smaller than the velocity dispersion near the central BH (a few hundreds $\mathrm{km} \mathrm{s}^{-1}$ ) and will eventually be disrupted through interactions with other stars. However, some small fraction may be hard enough to survive and evolve into compact binaries. Whether hard binaries will have an important dynamical role has to be explored. Their interaction with the central $\mathrm{BH}$ is of particular interest. Indeed, if it passes sufficiently close to the $\mathrm{BH}$, a binary will be tidally disrupted with the likely result of ejecting one star out of the cluster at very high velocity and leaving the other one bound to the BH (Hills 1988, 1991). This is another channel to form extreme mass ratio binaries to be detected by LISA.

6. Interaction with a central accretion disk or gas cloud. The early evolution of galactic nuclei may well lead to the accumulation of a quasi spherical central gas cloud with high enough a density to interact strongly with the stellar cluster. This situation has not yet been given the attention it deserves (see, however, 1992, and references therein) but further investigations have been undertaken by Amaro-Seoane and collaborators (Amaro-Seoane \& Spurzem 2001; Amaro-Seoane et al. 2001).

In AGNs, stars may be captured by an accretion disk through repeated impacts which can strongly reshape the stellar distribution in the vicinity of the BH (Norman \& Silk 1983; Syer et al. 1991; Rauch 1995; Vokrouhlický \& Karas 1998; Karas \& Šubr 2001; Vilkoviskij \& Czerny 2002). The further stellar and orbital evolution of the disk-embedded stars is a complex subject. Interesting possibilities include enhanced rate of collisions and growth of massive stars by accretion of disk material. Note that even if the interactions with the accretion disk are not efficient enough to grind down orbits into the disk, stellar formation probably occurs in situ (Goodman 2002) so that the presence of stars in the disk has to be expected anyway. A possible way of accounting for the role of the accretion disk in numerical models would be to use the MC code to simulate the outer quasi-spherical parts of the cluster where relaxation is important and couple it with a code like that of Šubr (2001) which treats the inner regions, where interactions with the disk dominate the dynamics, in axisymmetrical geometry.

7. Gas dynamics. Including stellar evolution without a better prescription for the fraction of gas that eventually finds its way to the central $\mathrm{BH}$ is nearly pointless, as demonstrated by simulations in Freitag (2000). Early studies (Bailey 1980; Loose \& Fricke 1980; David et al. 1987a,b; Kunze et al. 1987; Norman \& Scoville 1988) concluded that most of the gas finds its way to the central BH but they lacked detailed account of the feed-back on the gas of the energy released by the central source and supernova explosions and of the complex, non-spherical, evolving geometry of the gas flow (see, e.g., Williams et al. 1999; Ciotti \& Ostriker 2001, for recent attempts at tackling these intricacies).

This list can be lengthened virtually without end. But before we hurry and include more and more complexity in our simulations, we must keep in mind that each new process to be added comes with its own uncertainties of both physical and numerical nature, so that the impression of added "realism" may be misleading. In such a context, it is all the more useful to dispose of a numerical tool flexible enough to allow changes in the treatment of various physical effects and fast enough to allow large sets of simulations to be conducted to test for the influence of these modifications. 
Another line along which we have to progress is to develop definite observational predictions. Here are a few examples:

- surface luminosity and color profiles for central cusps;

- rate and characteristics of radiation flares following the tidal disruption of a star;

- appearance (and radial distribution) of stars modified by collisions or tidal interactions with the $\mathrm{MBH}$;

- rate and characteristics of gravitational waves signals from captured stars.

All examples but the first are complex problems of their own and have already been the subject of many detailed, if not conclusive, studies. Fortunately these aspects are essentially decoupled from the cluster dynamics, in the sense that they have no obvious back-influence on it, so that we should be able to "map" results from the literature on the outcome of our simulations.

Acknowledgements. Stellar models to initiate SPH collision simulations where kindly provided by the Geneva stellar evolution group, with precious help from Georges Meynet, and by Isabelle Baraffe and Corinne Charbonel. M.F. wants to thank Rainer Spurzem for interesting discussions and Gerald Quinlan and Roeland van der Marel for providing pycode, the $\mathrm{BH}$ adiabatic growth code. Comments by the anonymous referee helped to clarify the paper.

Most simulations have been realized on the "Beowulfs" clusters GRAVITOR at Geneva Observatory ${ }^{14}$, and ISIS at Bern University. This work has been supported by the Swiss National Science Foundation. The writing of this paper has been finished at Caltech, with partial support from NASA under grant NAG5-10707.

\section{Appendix A: Building of initial models of galactic nuclei}

To obtain initial cluster realizations for our simulations, we proceed in two stages: (1) We set the radii $R_{i}$, specific kinetic energies, $T_{i}$ and moduli of specific angular momentum, $J_{i}$ of all super-stars ${ }^{15}$ while trying to ensure dynamical equilibrium. (2) We set the stellar masses of the super-stars, $M_{i}^{*}$, according to a given initial mass function (IMF). To get an aged stellar population, we may also evolve this IMF according to the "ZAMS $\longrightarrow$ remnant" relation specified in Sect. 4.1. As the number of stars a super-star stands for must be the same for all super-stars, this stage also implicitly determines the superstar's mass, $M_{i}=\left(N_{*} / N_{\mathrm{p}}\right) M_{i}^{*}$ where $N_{\mathrm{p}}$ is the number of superstars the model consists of and $N_{*}$ is the number of stars represented by the model.

\section{A.1. Positions and velocities}

The safest way to obtain a system that is not only virialized $\left(2 T_{\mathrm{cl}}+U_{\mathrm{cl}}=0\right.$ where $T_{\mathrm{cl}}$ is the total kinetic energy and $U_{\mathrm{cl}}$ the total gravitational energy), but a genuine stationary solution of the collision-less Boltzmann equation, is to start from a

\footnotetext{
${ }^{14}$ http://obswww. unige.ch/ pfennige/gravitor/ gravitor_e.html

15 Remember that a super-star actually represents a spherical shell of stars.
}

one-particle DF $f(\boldsymbol{X}, \boldsymbol{V})$ which depends on the position $\boldsymbol{X}$ and velocity $\boldsymbol{V}$ only through isolating integrals of motions, namely $E$ and $J$, for a stellar cluster that obeys spherical symmetry (Binney \& Tremaine 1987, Chap. 4),

$$
f(\boldsymbol{X}, \boldsymbol{V})=F(E(\boldsymbol{X}, \boldsymbol{V}), J(\boldsymbol{X}, \boldsymbol{V})),
$$

with

$$
E(\boldsymbol{X}, \boldsymbol{V})=\frac{1}{2} V^{2}+\Phi(R) \text { and } J(\boldsymbol{X}, \boldsymbol{V})=R V_{\perp},
$$

where $R=|\boldsymbol{X}|, V=|\boldsymbol{V}|, V_{\perp}$ is the modulus of the component of $\boldsymbol{V}$ perpendicular to $\boldsymbol{X}$ (with the cluster center as origin of coordinates) and $\Phi$ is the (smooth) gravitational potential. For the sake of simplicity, we only considered initial cluster models with isotropic velocity distributions for which $F$ is a function of $E$ only. Note that our MC code can tackle any velocity distribution and that some level of anisotropy develops during the run of most cluster simulations.

$\Phi$ is itself determined by the DF through Poisson equation:

$2 \frac{\mathrm{d} \Phi}{\mathrm{d} R}+R \frac{\mathrm{d}^{2} \Phi}{\mathrm{d} R^{2}}=4 \pi G \rho(\Phi)$

with the density $\rho$ given by

$\rho(\Phi)=4 \pi \int_{0}^{\sqrt{-2 \Phi}} \mathrm{d} V V^{2} F\left(\frac{1}{2} V^{2}+\Phi\right)$.

It is customary to define so-called relative energy and potential through

$\Psi \stackrel{\text { def }}{=} \Phi_{0}-\Phi$ and $\varepsilon \stackrel{\text { def }}{=} \Phi_{0}-E$

with $\Phi_{0}$ chosen so that $F(\varepsilon)=0$ for $\varepsilon \leq 0$. For a cluster of finite radius $R_{\mathrm{cl}}, \Phi_{0}=-G M_{\mathrm{cl}} / R_{\mathrm{cl}}$.

Thus, to build a cluster model, we do the following:

(0) Choose an expression for $F(\varepsilon)$. Traditional choices are, among others, Plummer's or King's models (Binney \& Tremaine 1987).

(1) Integrate $\Psi(R)$ and $M_{\mathrm{r}}(R)$ with a Runge-Kutta scheme (Hairer et al. 1987):

$\frac{\mathrm{d}}{\mathrm{d} R}\left(\begin{array}{l}\Psi \\ \Psi_{\mathrm{d}} \\ M_{\mathrm{r}}\end{array}\right)=\left(\begin{array}{l}\Psi_{\mathrm{d}} \\ -4 \pi G \rho(\Psi)-\frac{2}{R} \Psi_{\mathrm{d}} \\ 4 \pi \rho(\Psi) R^{2}\end{array}\right)$.

Each evaluation of the function $\rho(\Psi)$ requires itself a numerical integration of Eq. (A.4). The integration of system (A.6) is terminated either when the relative potential reaches 0 (for tidally truncated models) or when $M_{\mathrm{r}}$ has attained some asymptotic value. At that point, we have obtained array representations of $R, \Psi, \rho$ and $M_{\mathrm{r}}$. We re-normalize them to the " $N$-body" system of units (see Sect. 1.3).

(2) For each super-star, radius $R_{i}$ is randomly selected according to the probability density $\mathrm{d} M_{\mathrm{r}} / \mathrm{d} R$. This is done by creating a random number $X_{\text {ran }}$ with uniform probability over [0; 1[ and (numerically) inverting the $M_{\mathrm{r}}(R)$ relation: $R_{i}=M_{\mathrm{r}}^{-1}\left(X_{\mathrm{ran}}\right)$. 
(3) Once the radius $R_{i}$ of super-star $i$ is determined, we have to select a velocity $V_{i}$ according to distribution $g(V) \propto$ $V^{2} F\left(\frac{1}{2} V^{2}+\Phi\left(R_{i}\right)\right)$. Here we use a simple rejection method (Press et al. 1992, Sect. 7.3) with a constant upper bound given by $-2 \Phi\left(R_{i}\right) F\left(\Phi\left(R_{i}\right)\right)^{16}$. The specific kinetic energy of the super-star is thus $T_{i}=V_{i}^{2} / 2$. To set the specific angular momentum $J_{i}$ with account of isotropy, we generate another random number $X_{\text {ran }}$ and compute $V_{\text {rad }}=V_{i}\left(1-2 X_{\text {ran }}\right)$ and $J_{i}=R_{i} \sqrt{V_{i}^{2}-V_{\mathrm{rad}}^{2}}$.

(4) Finally, perfect virial energy balance is enforced by a slight re-scaling of the velocities.

In its present form, this procedure does not explicitly allow for a central BH. But if we add such a point mass at the center with a very small mass (as compared to $M_{\mathrm{cl}}$ ), it will only slightly perturb the potential energies of the innermost superstars and the resulting system will still be very close to dynamical equilibrium. This is the reason why we must always start simulations with "seed" black holes instead of already grown (super-)massive ones. An advantage of this method is that the integrated influence of the BH's growth on the stellar system is "automatically" computed! The main drawback is that we cannot start with models that represent today's galactic nuclei but have to guess initial conditions that lead to such configurations after a Hubble time. This has not yet been explored systematically.

The cluster produced with this algorithm has no mass spectrum, i.e. all super-stars have the same mass $M_{\mathrm{p}}=M_{\mathrm{cl}} / N_{\mathrm{p}}$. We now explain how we construct a stellar mass spectrum.

\section{A.2. Masses}

We model IMFs that are piece-wise power-laws,

$\frac{\mathrm{d} N_{*}}{\mathrm{~d} M_{*}} \propto M_{*}^{-\alpha_{k}}$ for $M_{k-1} \leq M_{*} \leq M_{k}$,

between some $M_{0}=M_{\min }$ and $M_{K}=M_{\max }$.

For a given set of $M_{k}(k=0, \ldots, K)$ and $\alpha_{k}(k=1, \ldots, K)$. The un-normalized number of stars with masses $\leq M_{*}$ is, for $M_{k-1} \leq M_{*} \leq M_{k}$ :

$$
\begin{aligned}
N\left(M_{*}\right) & =N_{k-1}+C_{k} \int_{M_{k-1}}^{M_{*}} \frac{\mathrm{d} N_{*}}{\mathrm{~d} M_{*}} \mathrm{~d} M_{*} \\
& =N_{k-1}+\frac{1}{1-\alpha_{k}}\left(M_{*}^{1-\alpha_{k}}-M_{k-1}^{1-\alpha_{k}}\right)
\end{aligned}
$$

with $C_{k}=C_{k-1} M_{k-1}^{\left(\alpha_{k}-\alpha_{k-1}\right)}$ (we can set $C_{1}=1$ ). Once the $N_{k}$ have been computed, we randomly determine the stellar mass of each super-star in turn. We first generate a random number $N_{\text {ran }}$ with uniform $\left[0 ; N_{K}\right]$ distribution $\left(N_{K}\right.$ is the un-normalized total number). We then find index $j$ such that $N_{j-1} \leq N_{\text {ran }} \leq N_{j}$ and invert $N\left(M_{*}\right)$ to find the stellar mass for super-star $i$ :

$$
M_{i}^{*}=\left(M_{j-1}^{1-\alpha_{j}}+\left(1-\alpha_{j}\right) \frac{N_{\mathrm{ran}}-N_{j-1}}{C_{j}}\right)^{\frac{1}{1-\alpha_{j}}} .
$$

\footnotetext{
16 Bound particles have $V^{2} / 2+\Phi(R)<0$. Furthermore, well-behaved DF have $\mathrm{d} F / \mathrm{d} E<0$ so that the maximum value at a given $R$ is $F(\Phi(R))$.
}

Note that we never need to state the actual total number of stars (or, equivalently, the total mass in $M_{\odot}$ ) or the size of the cluster in pc when building initial models. This must only be specified before starting an evolutionary Monte Carlo simulation as these mass and size scales determine the relative importances of various processes (e.g. relaxation vs. collisions) and allows to translate the $N$-body time units into years.

\section{References}

Alexander, T. 1999, ApJ, 527, 835

Alexander, T., \& Kumar, P. 2001, ApJ, 549, 948

Alexander, T., \& Livio, M. 2001, ApJ, 560, L143

Amaro-Seoane, P., \& Spurzem, R. 2001, in The Central Kiloparsec of Starbursts and AGN: The La Palma Connection, ed. J. H. Knapen, J. E. Beckman, I. Shlosman, \& T. J. Mahoney, ASP Conf. Ser., 249, 731

Amaro-Seoane, P., Spurzem, R., \& Just, A. 2001, Super-massive stars: Dense star-gas systems, preprint, to appear in Lighthouses of the Universe [astro-ph/0110030]

Arabadjis, J. S. 1997, Ph.D. Thesis, University of Michigan

Ayal, S., Livio, M., \& Piran, T. 2000, ApJ, 545, 772

Bahcall, J. N., \& Wolf, R. A. 1976, ApJ, 209, 214

Bahcall, J. N., \& Wolf, R. A. 1977, ApJ, 216, 883

Bailey, M. E. 1980, MNRAS, 191, 195

Bailey, V. C., \& Davies, M. B. 1999, MNRAS, 308, 257

Baumgardt, H. 2001, MNRAS, 325, 1323

Begelman, M. C., Blandford, R. D., \& Rees, M. J. 1980, Nature, 287, 307

Begelman, M. C., \& Rees, M. J. 1978, MNRAS, 185, 847

Benz, W. 1990, in Numerical Modelling of Nonlinear Stellar Pulsations Problems and Prospects, ed. J. R. Buchler, 269

Benz, W., \& Hills, J. G. 1992, ApJ, 389, 546

Binney, J., \& Tremaine, S. 1987, Galactic Dynamics (Princeton University Press)

Bressan, A., Fagotto, F., Bertelli, G., \& Chiosi, C. 1993, A\&AS, 100, 647

Carter, B., \& Luminet, J.-P. 1983, A\&A, 121, 97

Chabrier, G., \& Baraffe, I. 2000, ARA\&A, 38, 337

Chandrasekhar, S. 1960, Principles of stellar dynamics (New York: Dover, Enlarged ed.)

Charbonnel, C., Däppen, W., Schaerer, D., et al. 1999, A\&AS, 135, 405

Chatterjee, P., Hernquist, L., \& Loeb, A. 2002, ApJ, 572, 371

Ciotti, L., \& Ostriker, J. P. 2001, ApJ, 551, 131

Cipollina, M. 1995, A\&AS, 110, 155

Cipollina, M., \& Bertin, G. 1994, A\&A, 288, 43

Claret, A., \& Gimenez, A. 1998, A\&AS, 133, 123

Cohn, H., \& Kulsrud, R. M. 1978, ApJ, 226, 1087

Colgate, S. A. 1967, ApJ, 150, 163

Combes, F. 2001, in Advanced Lectures on the Starburst-AGN Connection, INAOE, June 2000, ed. I. Aretxaga, R. Mújica, \& D. Kunth (World Scientific) [astro-ph/0010570]

Danzmann, K. 2000, Adv. Space Res., 25, 1129

David, L. P., Durisen, R. H., \& Cohn, H. N. 1987a, ApJ, 313, 556

David, L. P., Durisen, R. H., \& Cohn, H. N. 1987b, ApJ, 316, 505

Davies, M. B., Blackwell, R., Bailey, V. C., \& Sigurdsson, S. 1998, MNRAS, 301, 745

Dehnen, W. 1993, MNRAS, 265, 250

Di Stefano, R., Greiner, J., Murray, S., \& Garcia, M. 2001, ApJ, 551, L37

Duncan, M. J., \& Shapiro, S. L. 1982, ApJ, 253, 921 
Duncan, M. J., \& Shapiro, S. L. 1983, ApJ, 268, 565

Duquennoy, A., \& Mayor, M. 1991, A\&A, 248, 485

Einsel, C., \& Spurzem, R. 1999, MNRAS, 302, 81

Evans, C. R., \& Kochanek, C. S. 1989, ApJ, 346, L13

Ferrarese, L., Pogge, R. W., Peterson, B. M., et al. 2001, ApJ, 555, L79

Frank, J. 1978, MNRAS, 184, 87

Frank, J., \& Rees, M. J. 1976, MNRAS, 176, 633

Fregeau, J. M., Joshi, K. J., Portegies Zwart, S. F., \& Rasio, F. A. 2002, ApJ, 570, 171

Freitag, M. 2000, Ph.D. Thesis, Université de Genève

Freitag, M. 2001, Class. Quant. Grav., 18, 4033

Freitag, M., \& Benz, W. 2001, A\&A, 375, 711

Freitag, M., \& Benz, W. 2002a, A Comprehensive Set of Simulations of Collisions Between Main Sequence Stars in Galactic Nuclei, in preparation

Freitag, M., \& Benz, W. 2002b, in Stellar collisions \& mergers and their consequences., ed. M. Shara, ASP Conf. Ser., 263 [astro-ph/0101186]

Fukushige, T., \& Heggie, D. C. 2000, MNRAS, 318, 753

Fulbright, M. S. 1996, Ph.D. Thesis, University of Arizona

Gao, B., Goodman, J., Cohn, H., \& Murphy, B. 1991, ApJ, 370, 567

Gebhardt, K., Pryor, C., O'Connell, R. D., Williams, T. B., \& Hesser, J. E. 2000, AJ, 119, 1268

Genzel, R., Pichon, C., Eckart, A., Gerhard, O. E., \& Ott, T. 2000, MNRAS, 317, 348

Genzel, R., Thatte, N., Krabbe, A., Kroker, H., \& Tacconi-Garman, L. E. 1996, ApJ, 472, 153

Gerhard, O. E. 1994, in NATO ASIC Proc. 445: The Nuclei of Normal Galaxies: Lessons from the Galactic Center, ed. R. Genzel, \& A. I. Harris, 267

Ghez, A. M., Morris, M., Becklin, E. E., Tanner, A., \& Kremenek, T. 2000, Nature, 407, 349

Giersz, M. 1998, MNRAS, 298, 1239

Giersz, M. 2001, MNRAS, 324, 218

Giersz, M., \& Spurzem, R. 2000, MNRAS, 317, 581

Gold, T., Axford, W. I., \& Ray, E. C. 1965, in Quasi-stellar Sources and Gravitational Collapse, ed. I. Robinson, A. Schild, \& E. L. Schucking, 93

Goodman, J. 1983, ApJ, 270, 700

Goodman, J. 2002, Selfgravity and QSO disks, preprint [astro-ph/0201001]

Goodman, J., \& Binney, J. 1984, MNRAS, 207, 511

Gould, A., \& Rix, H. 2000, ApJ, 532, L29

Greiner, J., Schwarz, R., Zharikov, S., \& Orio, M. 2000, A\&A, 362, L25

Hairer, E., Nørsett, S. P., \& Wanner, G. 1987, Solving Ordinary Differential Equations I. Nonstiff Problems (Springer)

Hemsendorf, M., Sigurdsson, S., \& Spurzem, R. 2001, Collisional dynamics around binary black holes in galactic centers, preprint [astro-ph/0103410]

Hénon, M. 1959, Ann. Astrophys., 22, 126

Hénon, M. 1960, Ann. Astrophys., 23, 474

Hénon, M. 1960, Ann. Astrophys., 23, 668

Hénon, M. 1969, A\&A, 2, 151

Hénon, M. 1971a, Ap\&SS, 14, 151

Hénon, M. 1971b, Ap\&SS, 13, 284

Hénon, M. 1973, in Dynamical structure and evolution of stellar systems, Lectures of the 3rd Advanced Course of the Swiss Society for Astronomy and Astrophysics (SSAA), ed. L. Martinet, \& M. Mayor, 183

Hénon, M. 1975, in Dynamics of Stellar Systems, ed. A. Hayli, IAU Symp., 69, 133
Hernquist, L. 1990, ApJ, 356, 359

Hills, J. G. 1975, Nature, 254, 295

Hills, J. G. 1988, Nature, 331, 687

Hills, J. G. 1991, AJ, 102, 704

Hils, D., \& Bender, P. L. 1995, ApJ, 445, L7

Hughes, S. A. 2001a, Phys. Rev. D, 64, 4004

Hughes, S. A. 2001b, Class. Quant. Grav., 18, 4067

Ivanov, P. B. 2001, On formation rate of close binaries consisting of a super-massive black hole and a white dwarf, preprint [astro-ph/0112317]

Jaffe, W. 1983, MNRAS, 202, 995

Joshi, K. J., Nave, C. P., \& Rasio, F. A. 2001, ApJ, 550, 691

Joshi, K. J., Rasio, F. A., \& Portegies Zwart, S. 2000, ApJ, 540, 969

Karas, V., \& Šubr, L. 2001, A\&A, 376, 686

Kauffmann, G., \& Haehnelt, M. 2000, MNRAS, 311, 576

Kim, E., Einsel, C., Lee, H. M., Spurzem, R., \& Lee, M. G. 2002, MNRAS, 334, 310

Kim, S. S., \& Lee, H. M. 1999, A\&A, 347, 123

Kippenhahn, R., \& Weigert, A. 1994, Stellar Structure and Evolution (Springer-Verlag Berlin Heidelberg)

Komossa, S. 2001, X-ray Evidence for Supermassive Black Holes in Non-Active Galaxies, to appear in Lighthouses of the Universe, the most Luminous Celestial Objects and their use for Cosmology (Garching, Aug. 2001), ESO Astrophysics Symposia [astro-ph/0109441]

Kormendy, J., \& Gebhardt, K. 2001, in 20th Texas Symposium on relativistic astrophysics, ed. H. Martel, \& J. C. Wheeler, 363

Kunze, R., Loose, H.-H., \& Yorke, H. W. 1987, A\&A, 182, 1

Laguna, P., Miller, W. A., Zurek, W. H., \& Davies, M. B. 1993, ApJ, 410, L83

Lai, D., Rasio, F. A., \& Shapiro, S. L. 1993, ApJ, 412, 593

Lee, H. M., \& Ostriker, J. P. 1986, ApJ, 310, 176

Lee, M. H., \& Goodman, J. 1989, ApJ, 343, 594

Leeuwin, F., \& Athanassoula, E. 2000, MNRAS, 317, 79

Lidskii, V. V., \& Ozernoi, L. M. 1979, Sov. Astron. Lett., 5, 16

Lightman, A. P., \& Shapiro, S. L. 1977, ApJ, 211, 244

Lin, D. N. C., \& Tremaine, S. 1980, ApJ, 242, 789

Lombardi, J. C., Warren, J. S., Rasio, F. A., Sills, A., \& Warren, A. R. 2002, ApJ, 568, 939

Loose, H.-H., \& Fricke, K. J. 1980, Astron. Lett., 21, 65

Lynden-Bell, D. 1969, Nature, 223, 690

MacMillan, J. D., \& Henriksen, R. N. 2002, ApJ, 569, 83

Maeder, A. 1992, A\&A, 264, 105

Magorrian, J., \& Tremaine, S. 1999, MNRAS, 309, 447

Makino, J. 2001, in Dynamics of Star Clusters and the Milky Way, ed. S. Deiters, B. Fuchs, R. Just, \& R. Spurzem, ASP Conf. Ser., 228,87

McMillan, S. L. W., Lightman, A. P., \& Cohn, H. 1981, ApJ, 251, 436

McMillan, S. L. W., McDermott, P. N., \& Taam, R. E. 1987, ApJ, 318 , 261

Menou, K., Haiman, Z., \& Narayanan, V. K. 2001, ApJ, 558, 535

Menou, K., \& Quataert, E. 2001, ApJ, 562, L137

Merritt, D., \& Cruz, F. 2001, ApJ, 551, L41

Merritt, D., \& Ferrarese, L. 2001, ApJ, 547, 140

Meynet, G., Maeder, A., Schaller, G., Schaerer, D., \& Charbonnel, C. 1994, A\&AS, 103, 97

Milosavljević, M., \& Merritt, D. 2001, ApJ, 563, 34

Miralda-Escudé, J., \& Gould, A. 2000, ApJ, 545, 847

Murphy, B. W., \& Cohn, H. N. 1988, MNRAS, 232, 835

Murphy, B. W., Cohn, H. N., \& Durisen, R. H. 1991, ApJ, 370, 60

Nakano, T., \& Makino, J. 1999, ApJ, 525, L77

Norman, C., \& Scoville, N. 1988, ApJ, 332, 124

Norman, C., \& Silk, J. 1983, ApJ, 266, 502 
Novikov, I. D., Pethick, C. J., \& Polnarev, A. G. 1992, MNRAS, 255, 276

Peebles, P. J. E. 1972, ApJ, 178, 371

Phinney, E. S. 1989, in The Center of the Galaxy, ed. M. Morris, IAU Symp., 136, 543

Polnarev, A. G., \& Rees, M. J. 1994, A\&A, 283, 301

Poon, M. Y., \& Merritt, D. 2002, ApJ, 568, L89

Press, W. H., \& Teukolsky, S. A. 1977, ApJ, 213, 183

Press, W. H., Teukolsky, S. A., Vetterling, W. T., \& Flannery, B. P. 1992, Numerical Recipies in FORTRAN (Cambridge University Press)

Quinlan, G. D., Hernquist, L., \& Sigurdsson, S. 1995, ApJ, 440, 554

Quinlan, G. D., \& Shapiro, S. L. 1990, ApJ, 356, 483

Rasio, F. A., Fregeau, J. M., \& Joshi, K. J. 2001, in The influence of binaries on stellar population studies, Astrophysics and space science library (ASSL), vol. 264 (Kluwer Academic Publishers), 387

Rauch, K. P. 1995, MNRAS, 275, 628

Rauch, K. P. 1999, ApJ, 514, 725

Rees, M. J. 1988, Nature, 333, 523

Renzini, A. 2001, in Black Holes in Binaries and Galactic Nuclei. Proc. of the ESO Workshop held at Garching, Germany, 6-8 September 1999, ed. L. Kaper, E. P. J. van den Heuvel, \& P. A. Woudt (Springer), 161

Sanders, R. H. 1970, ApJ, 162, 791

Sanders, R. H., \& van Oosterom, W. 1984, A\&A, 131, 267

Schaller, G., Schaerer, D., Meynet, G., \& Maeder, A. 1992, A\&AS, 96,269

Sembay, S., \& West, R. G. 1993, MNRAS, 262, 141

Shapiro, S. L. 1985, in Dynamics of Star Clusters, ed. J. Goodman, \& P. Hut, IAU Symp., 113, 373

Shapiro, S. L., \& Teukolsky, S. A. 1983, Black holes, white dwarfs, and neutron stars: The physics of compact objects (WileyInterscience)

Shields, G. A., \& Wheeler, J. C. 1978, ApJ, 222, 667

Shlosman, I. 1992, in Relationships Between Active Galactic Nuclei and Starburst Galaxies, ASP Conf. Ser., 31, 335

Shlosman, I., Begelman, M. C., \& Frank, J. 1990, Nature, 345, 679

Sigurdsson, S., Hernquist, L., \& Quinlan, G. D. 1995, ApJ, 446, 75

Sigurdsson, S., \& Rees, M. J. 1997, MNRAS, 284, 318

Sills, A., Faber, J. A., Lombardi, J. C., Rasio, F. A., \& Warren, A. R. 2001, ApJ, 548, 323

Sills, A., Lombardi, J. C., Bailyn, C. D., et al. 1997, ApJ, 487, 290
Spitzer, L. J., \& Saslaw, W. C. 1966, ApJ, 143, 400

Spitzer, L. 1987, Dynamical evolution of globular clusters (Princeton University Press)

Spitzer, L. J., \& Stone, M. E. 1967, ApJ, 147, 519

Spurzem, R. 1992, in Rev. Mod. Astron., 5, 161

Spurzem, R., \& Kugel, A. 2000, in Molecular Dynamics on Parallel Computers, ed. R. Esser, P. Grassberger, J. Grotendorst, \& M. Lewerenz (World Scientific), 264

Stodołkiewicz, J. S. 1982, Acta Astron., 32, 63

Stodołkiewicz, J. S. 1986, Acta Astron., 36, 19

Šubr, L. 2001, Ph.D. Thesis, Charles University Prague

Syer, D., Clarke, C. J., \& Rees, M. J. 1991, MNRAS, 250, 505

Syer, D., \& Ulmer, A. 1999, MNRAS, 306, 35

Takahashi, K., \& Portegies Zwart, S. F. 2000, ApJ, 535, 759

Thorne, K. S. 1998, in Black Holes and Relativistic Stars, ed. R. M. Wald, 41

Torricelli-Ciamponi, G., Foellmi, C., Courvoisier, T. J.-L., \& Paltani, S. 2000, A\&A, 358, 57

Tremaine, S., Richstone, D. O., Byun, Y., et al. 1994, AJ, 107, 634

Ulam, S. M., \& Walden, W. E. 1964, Nature, 201, 1202

van der Marel, R. P. 1999, AJ, 117, 744

van der Marel, R. P. 2001, in Black Holes in Binaries and Galactic Nuclei, Proc. of the ESO Workshop held at Garching, Germany, 6-8 September 1999, ed. L. Kaper, E. P. J. van den Heuvel, \& P. A. Woudt, ESO Astrophysics Symp. (Berlin HeidelBerg New York: Springer), 246

Vilkoviskij, E. Y., \& Czerny, B. 2002, A\&A, 387, 804

Vokrouhlický, D., \& Karas, V. 1998, MNRAS, 293, L1

Volonteri, M., Haardt, F., \& Madau, P. 2002, The Assembly and Merging History of Supermassive Black Holes in Hierarchical Models of Galaxy Formation, preprint [astro-ph/0207276]

von Hoerner, S. 1968, in Colloque sur le problème des $N$ corps, CNRS, 147

Watters, W. A., Joshi, K. J., \& Rasio, F. A. 2000, ApJ, 539, 331

Williams, R. J. R., Baker, A. C., \& Perry, J. J. 1999, MNRAS, 310, 913

Woltjer, L. 1964, Nature, 201, 803

Young, P. J. 1977, ApJ, 215, 36

Young, P. J. 1980, ApJ, 242, 1232

Young, P. J., Shields, G. A., \& Wheeler, J. C. 1977, ApJ, 212, 367

Yu, Q. 2002, MNRAS, 331, 935

Zhao, H.-S., Haehnelt, M. G., \& Rees, M. J. 2002, New Astron., 7 , 385 